COORDINATION OF DISTRIBUTED MPC SYSTEMS THROUGH DYNAMIC REAL-TIME OPTIMIZATION WITH CLOSED-LOOP PREDICTION 


\title{
COORDINATION OF DISTRIBUTED MPC SYSTEMS THROUGH DYNAMIC REAL-TIME OPTIMIZATION WITH CLOSED-LOOP PREDICTION
}

by

Hao Li, B.Eng

\author{
A Thesis \\ Submitted to the School of Graduate Studies \\ in Partial Fulfillment of the Requirements \\ for the Degree \\ Master of Applied Science
}

McMaster University

(c) Copyright by Hao Li, April 2018 
MASTER OF APPLIED SCIENCE (2018)

(Chemical Engineering)
McMaster University

Hamilton, Ontario, Canada

TITLE:

Coordination of Distributed MPC Systems through

Dynamic Real-Time Optimization with Closed-Loop Prediction

AUTHOR:

Hao Li, B.Eng

(McMaster University, Hamilton, ON)

SUPERVISOR:

Dr. Christopher L.E. Swartz

NUMBER OF PAGES:

xiii 116 


\begin{abstract}
A dynamic real-time optimization (DRTO) formulation with closed-loop prediction is used to coordinate distributed model predictive controllers (MPCs) by rigorously predicting the interaction between the distributed MPCs and full plant response in the DRTO formulation. This results a multi-level optimization problem and that is solved by replacing the MPC quadratic programming subproblems by their equivalent Karush-Kuhn-Tucker (KKT) first-order optimality conditions to yield a single-level mathematical program with complementarity constraints (MPCC). The proposed formulation is able to perform both target tracking and economic optimization with significant performance improvement over decentralized control, and similar performance to centralized MPC. A linear dynamic case study illustrates the performance of the proposed strategy for coordination of distributed MPCs for different levels of plant interaction,. The method is thereafter applied to a nonlinear integrated plant with recycle, where its performance in both set-point target tracking and economic optimization is demonstrated.
\end{abstract}

Subsequently, this study presents two techniques for approximation of the closed-loop prediction within the DRTO formulation - a hybrid closed-loop formulation and an input clipping formulation. The hybrid formulation generates closed-loop predictions for a limited number of time intervals along the DRTO prediction horizon, followed by an open-loop optimal control formulation extended to rest of the horizon. The input clipping formulation utilizes an unconstrained MPC optimization formulation for each distributed MPC, coupled with the application of an input saturation mechanism. The performance of the approximation techniques is evaluated through application to case studies based on linear and nonlinear dynamic plant models respectively. The approximation techniques are demonstrated to be more computationally efficient than than the rigorous counterpart without significant loss in performance. 
The performance of the proposed DRTO formulation can be further improved by the introduction of nonlinearity. The nonlinear dynamic plant model is firstly introduced in the DRTO formulation while maintaining the linear formulation for the distributed MPCs. The performance of resulting formulation is demonstrated and compared against the linear counterpart. The nonlinear MPC formulation is then included in both lower-level control implementation and DRTO formulation. By reformulating the Lagrangian of the nonlinear MPC optimization subproblems, the nonlinear MPC formulation is successfully implemented in the DRTO formulation. The performance of such DRTO formulation is further improved and shown using a nonlinear case study.

The conclusion of this study is summarized and the potential directions of this research such as large-scale applications, variation of MPC implementations, and robust model-based control are outlined and explained in the end. 


\section{ACKNOWLEDGEMENTS}

I would like to express my immense gratitude towards my academic supervisor, Dr. Christopher L.E. Swartz, for being an amazing mentor throughout my undergraduate and graduate studies at McMaster University. His unqualified academic, personal and financial support assisted me to accomplish more than what I could imagine prior to my studies. I would also like to acknowledge the generous support from McMaster University and McMaster Advanced Control Consortium (MACC) as institutions for providing me many great opportunities in terms of scholarships and resources.

My appreciation also extends to my loving parents who overcame tremendous struggles to provide me high-quality education, a loving family, and chances to broaden my vision and to grow as a person, and to my friends for their companionship, advices

and precious memories. It is them who made my years in Canada so endearing and memorable. 


\section{Table of Contents}

$\begin{array}{lll}1 & \text { Introduction } & 1\end{array}$

\begin{tabular}{|lll}
2 & Literature Review & 4
\end{tabular}

2.1 Distributed model predictive control $\ldots \ldots \ldots \ldots \ldots$

2.2 Dynamic real-time optimization $\ldots \ldots \ldots \ldots \ldots \ldots$

\begin{tabular}{lll}
\hline 3 & Rigorous DRTO Formulation & 10
\end{tabular}

$3.1 \quad$ Closed-loop DRTO (CL-DRTO) formulation for distributed MPC sys-

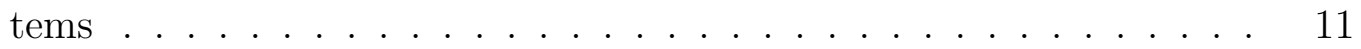

3.1.1 Preliminaries: Full-scale and local plant models . . . . . . . 11

3.1 .2 MPC formulation . . . . . . . . . . . . . . . . . 13

$3.1 .3 \quad$ Optimization formulation $\ldots \ldots \ldots \ldots$

$3.1 .4 \quad$ Solution approach $\ldots \ldots \ldots \ldots \ldots$

3.1 .5 Implementation variants $\ldots \ldots \ldots \ldots \ldots \ldots \ldots$

3.2 Case Studies . . . . . . . . . . . . . . . . . . . . . . . . . . . 23 
$3.2 .1 \quad$ Case Study 1: Linear Dynamic System . . . . . . . . . . . . 23

3.2 .2 Case Study 2: Dynamic optimization on a nonlinear integrated plant . . . . . . . . . . . . . . . . . . . 31

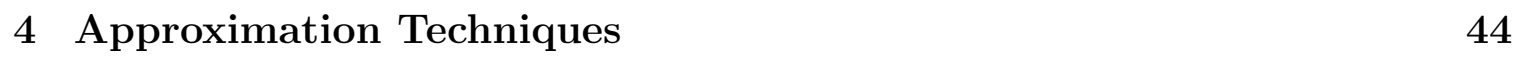

4.1 Closed-loop DRTO formulation $\ldots \ldots \ldots \ldots$

$4.1 .1 \quad$ Preliminaries: Full-scale and local plant models . . . . . . . 46

4.1 .2 Rigorous CL-DRTO formulation . . . . . . . . . . . . . 47

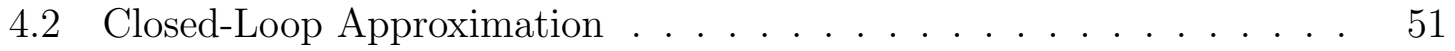

4.2 .1 Hybrid CL-DRTO formulation . . . . . . . . . . . . 51

4.2 .2 Input clipping formulation $\ldots \ldots \ldots \ldots \ldots \ldots$

$4.3 \quad$ Solution approach $\ldots \ldots \ldots \ldots \ldots \ldots$

$4.3 .1 \quad$ Karush-Kuhn-Tucker(KKT) optimality conditions . . . . . . 57

4.3 .2 Analytical solution of unconstrained MPC problem . . . . . 58

$4.4 \quad$ Case Studies . . . . . . . . . . . . . . . . . . . . . . . . . . . . . . . . . . . . . . . . . . . 60

$4.4 .1 \quad$ Target tracking for a linear transfer function system . . . . . . 61

4.4.2 $\quad$ Dynamic optimization of a nonlinear system of two CSTRs in series . . . . . . . . . . . . . . . . 70

\begin{tabular}{|lll}
\hline 5 & Inclusion of Nonlinearity & 81
\end{tabular} 
5.1 Problem Formulation for the Inclusion of Nonlinear Dynamic Plant Model . . . . . . . . . . . . . . . . . . . . . 82

5.1 .1 Model Discretization . . . . . . . . . . . . . . . . . . . . . 82

5.1 .2 State and Disturbance Estimation for Nonlinear Plant Model . 84

$5.1 .3 \quad$ Optimization Formulation . . . . . . . . . . . . . 85

5.1 .4 Case Study . . . . . . . . . . . . . . . . . . . . . 87

5.2 Problem Formulation for Coordination of Nonlinear MPCs . . . . . . 90

5.2 .1 Model Discretization . . . . . . . . . . . . . . . . . . . . . . . 92

5.2.2 State and Disturbance Estimation for Nonlinear Plant Model . 93

$5.2 .3 \quad$ Optimization Formulation . . . . . . . . . . . . . . . . . 95

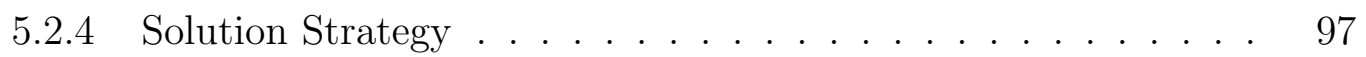

5.3 Case Study . . . . . . . . . . . . . . . . . . . . . . . . . . . . . . . . 98

5.3 .1 Target Tracking . . . . . . . . . . . . . . . . . 102

5.3 .2 Economic Optimization . . . . . . . . . . . . . . . 104

6 Conclusions and Recommendations 107

6.1 Conclusions . . . . . . . . . . . . . . . . . . . . . . 107

6.2 Recommendations for Further Work . . . . . . . . . . . . . . . . . . . 109

$\begin{array}{ll}\text { References } & 111\end{array}$ 


\section{List of Figures}

3.1 The control architecture for coordination of distributed MPCs with CL-DRTO approach. . . . . . . . . . . . . . . . . . . . . . . 12

3.2 The illustration of set-point extraction from reference trajectory. . . . 18

3.3 Output response under centralized and decentralized MPC alone. Dotdashed line: centralized MPC, Solid line: decentralized MPC, Dashed line: set-point. . . . . . . . . . . . . . . . . . . . 26

$3.4 \quad$ Output response (solid line) with set-points (dashed line) under distributed MPC coordinated by DRTO layer with no SPH, and output response (dot-dashed line) under centralized MPC. . . . . . . . . 27

$3.5 \quad$ Output response (solid line) with set-points (dashed line) under distributed MPCs coordinated by DRTO layer with $\mathrm{SPH}=3$, and output response (dot-dashed line) under centralized MPC. . . . . . . . . . . 29

$3.6 \quad$ Process schematic of the integrated plant. $\ldots \ldots \ldots \ldots$

$3.7 \quad$ Output responses under distributed MPC with DRTO layer for target

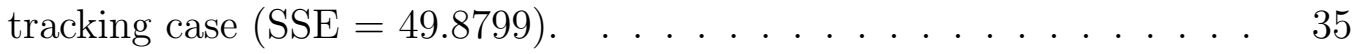


3.8 Input responses under distributed MPC with DRTO layer for target tracking case $(\mathrm{SSE}=49.8799)$. . . . . . . . . . . . . . . . . . . . . 35

3.9 Output responses under centralized MPC alone for target tracking case $(\mathrm{SSE}=79.7913) . \ldots \ldots \ldots$

3.10 Input responses under centralized MPC alone for target tracking case $(\mathrm{SSE}=79.7913) . \ldots \ldots \ldots \ldots$

3.11 Output responses under decentralized MPC for target tracking case $(\mathrm{SSE}=84.3017)$. . . . . . . . . . . . . . . . . . . . . . . . 37

3.12 Input responses under decentralized MPC for target tracking case (SSE $=84.3017$. . . . . . . . . . . . . . . . . . . 38

3.13 Output responses under distributed MPC with DRTO formulation for economic case $($ profit $=\$ 66,113)$. . . . . . . . . . . . . . . . . . . 42

3.14 Input responses under distributed MPC with DRTO formulation for economic case $($ profit $=\$ 66,113)$. . . . . . . . . . . . . . . . . 43

4.1 The control architecture for coordination of distributed MPCs with CL-DRTO approach . . . . . . . . . . . . . . . . . . 45

4.2 The illustration of set-point extraction from reference trajectory. . . . 50

4.3 Output responses with set-point trajectories for the rigorous case . . 65

$4.4 \quad$ Input responses with set-point trajectories for the rigorous case . . . 66

4.5 Output responses with set-point trajectories for the hybrid case . . . 67

$4.6 \quad$ Input responses with set-point trajectories for the hybrid case . . . . 68 
4.7 The problem sizes with increasing control horizon $\ldots \ldots \ldots$

4.8 The problem sizes for hybrid formulation with increasing closed-loop prediction horizon $\ldots \ldots \ldots \ldots$. . . . . . . . . . . 70

4.9 Schematics of the two CSTRs in series $\ldots \ldots \ldots \ldots . \ldots 71$

4.10 The output responses with setpoint trajectories for the hybrid with clipping case . . . . . . . . . . . . . . . . . . . 76

4.11 The input responses with setpoint trajectories for the hybrid with clipping case . . . . . . . . . . . . . . . . . . . . . . . 77

5.1 The control architecture for coordination of distributed MPCs with CL-DRTO approach. . . . . . . . . . . . . . . . . . . 83

5.2 Set-points and output response for distributed MPC cases . . . . . . 90

5.3 The control architecture for coordination of distributed MPCs with closed-loop DRTO approach. . . . . . . . . . . . . . . . . . . . . . . . 91

5.4 The set-point trajectory extractions from the reference trajectory. . . 96

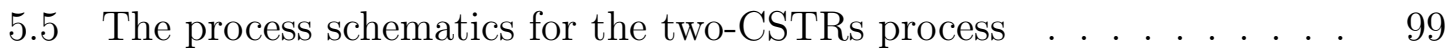

5.6 The plant response and set-point trajectories for target tracking . . . 103

5.7 The plant response and set-point trajectories for economic optimization 106 


\section{List of Tables}

3.1 Design parameters for the DRTO and MPC layers in Case Study 1. . 24

3.2 Performance for configurations in Case 1 in terms of sum of squared errors $(\mathrm{SSE})$.

3.3 Performance for configurations in Case Study 1 in terms of sum of squared errors (SSE) for different interaction scaling factors $\alpha$. . . . . 30

$3.4 \quad$ Process parameters for the integrated plant. . . . . . . . . . . . . . 33

$3.5 \quad$ Initial steady-state values for inputs and outputs of the integrated plant. 33

$3.6 \quad$ Bounds for inputs and outputs of the integrated plant. . . . . . . . . 34

3.7 Design parameters for the DRTO and MPC layers for the target tacking case in Case Study 2. . . . . . . . . . . . . . . . . . . . . . . . . . . . 39

$3.8 \quad$ Performance of each scenario for the target tracking case. . . . . . . . 39

3.9 Design parameters for the DRTO and MPC layers for the economic case in Case Study 2. . . . . . . . . . . . . . . . . . . . . . . . . . . 41

4.1 Design parameters for the DRTO and MPC layers for the linear transfer function system . . . . . . . . . . . . . . . . 63 
4.2 Summary of performance for the linear transfer function system . . . 64

4.3 Design parameters for the DRTO and MPC layers for the two-CSTR system . . . . . . . . . . . . . . . . . . . . . . 74

$4.4 \quad$ Initial steady-state values for inputs and outputs of the integrated plant. 74

4.5 The process results for the two-CSTR system . . . . . . . . . . 75

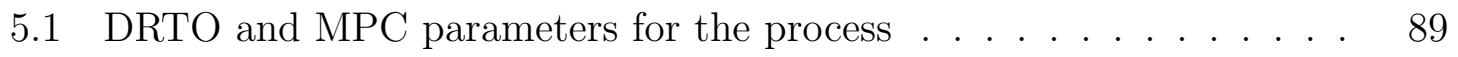

5.2 The summary of performance under different configurations . . . . . 91

$5.3 \quad$ DRTO and MPC parameters for the process . . . . . . . . . . . . 101

5.4 The summary of SSE values for three cases _ . . . . . . . . . . . . . 104

5.5 The summary of profits for three cases . . . . . . . . . . . . . . . . . 104 


\section{Chapter 1}

\section{Introduction}

A centralized model predictive control (MPC) system that considers all control actions simultaneously within a single optimization problem can be adopted for achieving optimal process operation. However, a collection of MPC controllers is usually implemented for large scale systems such as oil refineries and chemical plants, each of which is responsible for the control performance of an associated subsystem Qin and Badgwell, 2003, Scattolini, 2009. Distributed MPC systems aim to provide equivalent performance to that of a centralized counterpart, with additional flexibility and reliability brought by the independence of each controller [Pannocchia, 2013].

In decentralized control, plant subsystems are independently controlled with no communication between or coordination of the individual MPC subsystems. Failure to recognize interaction between plant subsystems leads to performance degradation, particularly when these interactions are strong. Distributed control architectures, on the other hand, involve communication between the MPC subsystems and can take different forms, such as use of a common MPC objective function (cooperative) or use of independent local performance objectives (noncooperative). While the MPC formulations may model the effects of inputs manipulated by other MPC subsystems, 
each MPC subsystem calculates and manipulates only the subset of inputs with which it is associated. The MPC systems can alternatively be coordinated through a higherlevel supervisory system in a hierarchical architecture. A brief description of these distributed control paradigms is provided in the next section; comprehensive reviews are given in Scattolini 2009]; Christofides et al. [2013]; Pannocchia 2013].

This thesis presents a strategy for coordination of distributed MPC systems following a hierarchical approach, through an upper-level dynamic real-time optimization (DRTO) system. The DRTO system communicates with the underlying MPC subsystems through set-point trajectories that are calculated based on the prediction of the closed-loop response of the plant under the distributed MPC system. The MPC subsystems do not communicate directly with each other; they represent standard MPC formulations with local dynamic models. We consider DRTO objectives based on plant economics or set-point target tracking. This work extends the DRTO closedloop prediction paradigm proposed in Jamaludin and Swartz 2017b a to distributed MPC systems.

Due to the inclusion of closed-loop prediction in the DRTO formulation, the issue of computational complexity brought by problem size and large control horizon needs to be addressed. Two approximation techniques are developed to reformulate the rigorous DRTO formulation while preserving the performance. The approximation techniques serve as extensions based on the work proposed by Jamaludin and Swartz $2017 \mathrm{a}$.

In order to further improve the performance, nonlinear dynamic plant model is subsequently incorporated in the DRTO formulation to provide more accurate surrogate plant response for the closed-loop prediction. The ordinary differential equations (ODEs) are discretized using the implicit Euler approximation. While other discretization methods such as orthogonal collocation and direct multiple shooting can be used, the Euler approximation is shown to be sufficient for the case study consid- 
ered.

The coordination scheme is not limited to linear MPC formulations. If the lower level MPCs are nonlinear MPCs (NMPCs), the DRTO formulation can be also easily adopted. The nonlinear formulation of MPCs can be included in the DRTO formulation through use of the Lagrangian. By using the Karush-Kuhn-Tucker (KKT) optimality conditions, the NMPC problem can be transformed into sets of algebraic equations with appropriate Jacobian calculations. With both nonlinear dynamic plant model and nonlinear MPCs, the DRTO formulation exhibits even better performance for the case study considered.

The remainder of this thesis is organized as follows. Chapter 2 presents a brief review of literature on different paradigms for coordination of distributed MPC systems and recent developments in the area of DRTO. Chapter 3 presents the detailed DRTO coordination scheme with solution approach and implementation variants, along with linear and nonlinear case studies to demonstrate the use of the DRTO formulation. Chapter 4 presents the approximation techniques applied to the DRTO formulation and demonstrates the improvement in terms of computational complexity for such reformulations. Chapter 5 introduces various degrees of nonlinearity to the DRTO formulation and control implementations and the further improvement of performances are illustrated using nonlinear case studies. Chapter 6 gives a summary of conclusions and contributions and provides a few future directions to be considered in subsequent research endeavors. 


\section{Chapter 2}

\section{Literature Review}

This chapter aims to provide a brief literature review on the concepts of distributed model predictive control (MPC) and real-time optimization (RTO) so that the essential background can be effectively illustrated and the novelty of this study can be shown by comparison.

\subsection{Distributed model predictive control}

Large-scale industrial systems typically employ MPC systems in a decentralized configuration, largely for reasons of maintainability and reliability Scheu and Marquardt, 2011; Stewart et al. 2010. Decentralized control, in which there is no communication between subsystems either directly or through a coordinator, can result in poor performance and instability in the presence of strong interactions. Different methodologies have been developed to address this issue, with communication-based and coordination-based MPC strategies being the two major paradigms. Some interesting work on this topic is summarized in Maestre et al. 2014. 
The communication-based MPCs can be configured to either allow the cooperation among MPCs under a global objective function, or only permit essential parameters to be exchanged among controllers which do not share the same objective. Camponogara et al. 2002 describe a noncooperative MPC scheme in which local MPC subsystems are solved in an iterative manner utilizing information from their neighbors, and state conditions under which the scheme converges to an equivalent centralized MPC problem. They also provide stability conditions for a scheme in which the local subsystems communicate their solutions only once during each control interval. Venkat et al. [2008] consider both noncooperative and cooperative distributed MPC schemes; the former utilizes local objective functions and upon convergence yields a Nash equilibrium, while in the latter, the local MPCs utilize a common objective function. The general case involves iteration with exchange of information between local controllers within each sample time. However, stability of the cooperative scheme is established for termination of iterations prior to convergence. Stewart et al. [2010] prove stability of cooperative MPC as a subcase of suboptimal MPC. They also consider the stability of output feedback cooperative MPC with estimation error. Sun and El-Farra 2008 consider a quasi-decentralized control system in which communication between local controllers is limited. Linear state feedback is assumed, and the maximum allowable information update period for closed-loop stability is characterized. Chen et al. 2012 propose a distributed Lyapunov-based economic MPC (LEMPC) scheme in which the local controller inputs are sequentially computed. Stability of the method is established, and its performance compared to that of centralized EMPC through application to a chemical process network case study. Farina and Scattolini [2012 present a noncooperative distributed MPC scheme in which local MPC subsystems do not require dynamic models of other subsystems, and require only state information from their neighbors. State and input constraints are accommodated, and stability is proved under stated conditions. Razzanelli and Pannocchia 2016 propose a cooperative distributed MPC scheme in which local MPC subsystems do 
not require information on the full plant state, resulting in reduced computational and communication requirements.

The coordination-based MPCs contain a coordinator used to process information collected from local controllers or share certain information for the purpose of coordination. Scheu and Marquardt 2011] present a sensitivity-based coordination scheme for distributed MPC systems. The solution of a centralized optimization problem is sought, whose objective function is separable in the local objective functions. The local objective functions are augmented with a term containing first-order sensitivity information from the other subproblems. Iteration is shown to converge to the solution of the centralized problem. Marcos et al. [2014 present a coordination scheme developed for linear discrete-time unconstrained systems. A coordinator provides predicted states of the full plant and price information to local MPCs. Iteration is shown to converge to the solution of the centralized problem. Closed-loop stability is analyzed, including for the case in which iterations are terminated prior to convergence. Farina et al. 2017 propose a hierarchical control scheme in which a higher level controller utilizes a reduced-order model, and is executed at a lower frequency than local MPCs, with combined inputs applied to the process. A robust MPC formulation is used at the upper level, and convergence analysis of the overall system is provided. Mart et al. 2013 present a price coordination scheme that calculates the shared resources to be assigned to lower-level NMPC controllers. The lower-level controllers incorporate the price information in the objective function. The overall performance approximates the centralized counterpart with shorter computational time. The proposed control structure is applied to an oxygen supply system to reactors. 


\subsection{Dynamic real-time optimization}

Process plant automation systems have traditionally followed a hierarchical configuration in which an upper level RTO system provides set-points to an underlying MPC layer Qin and Badgwell, 2003]. Industrial RTO systems have conventionally adopted steady-state models for the economic optimization Marlin and Hrymak, 1997; Darby et al. 2011. However, this assumption delays the economic evaluations until steady-state operation has been reached, which poses a significant drawback for processes exhibiting slow dynamics and frequent plant transitions. This has motivated the development of strategies in which plant dynamics are considered in the economic optimization calculation. Two key paradigms are a hierarchical approach in which a dynamic plant model is used at the upper economic level (dynamic RTO or DRTO) Tosukhowong et al., 2004, Kadam et al., 2002, and a single-level economic MPC (EMPC) approach in which economics and regulation are simultaneously addressed [Amrit et al., 2011; Heidarinejad et al., 2012]. In this section, we focus on the former due to its close connection to the proposed formulation. A critical review of feedback control structures for optimal process operation is given in Engell [2007], and a comprehensive review of EMPC is given in Ellis et al. 2014.

Kadam et al. 2002 propose a two-level DRTO structure with nonlinear dynamic models at both the upper, economic optimization and lower, regulatory control levels. The DRTO calculation is triggered based a disturbance sensitivity analysis. The method is demonstrated through application to a semi-batch reactive distillation case study. In Würth et al. [2011], a similar structure is adopted, with the lower level control inputs updated based on sensitivity calculations. Tosukhowong et al. 2004 regulate multiple MPC subsystems using the DRTO strategy in which a reduced-order model designed to capture the slow dynamics is used at the upper, economic level, and is executed at a lower frequency than that of the lower level MPCs. The feasibility of the set-point trajectories generated by the DRTO layer is ensured by placing a coor- 
dination layer between the DRTO layer and the MPCs. Ellis and Christofides 2014] propose a two-layer structure, with an upper level EMPC generating economically optimal state trajectories for a lower level Lyapunov MPC to track. A stability analysis is conducted, and the performance of the method demonstrated through application to a non-isothermal CSTR. The above-described two-level DRTO approaches utilize an open-loop prediction of the plant response, in contrast to the formulation described next, and on which the present study is based. However, these latter formulations do not directly include stability conditions, as in Ellis and Christofides 2014.

Jamaludin and Swartz 2017b propose a DRTO strategy in which the upper economic layer utilizes the predicted closed-loop response of the plant under the action of a single MPC system. This is shown to yield superior performance over prior approaches based on open-loop plant dynamics, particularly when the MPC is detuned such as in the presence of nonminimum phase characteristics. The DRTO formulation results in a multilevel optimization problem due to the sequence of MPC quadratic programming (QP) subproblems to generate the closed-loop response. This problem is reformulated as a single-level mathematical program with complementarity constraints (MPCC) by replacing the inner QP subproblems by their first-order optimality conditions. Jamaludin and Swartz 2017a propose three alternative formulations for approximating the closed-loop dynamics, resulting in significantly reduced computation times with little degradation in performance. The framework is shown in Jamaludin and Swartz 2016] to readily accommodate a nonlinear DRTO plant model.

In this study, a hierarchical DRTO framework is utilized for coordination of distributed MPC systems. The DRTO calculation is based on the prediction of the closed-loop response of the plant under the action of the local MPCs, thereby accounting for the interaction between subsystems. Communication from the DRTO level to the local MPCs is through set-point trajectories, with no exchange of in- 
formation between local controllers and no iteration between the DRTO and MPC levels within the DRTO sample time. The solution strategies and variation of the formulations are introduced in the subsequent chapters. 


\section{Chapter 3}

\section{Rigorous DRTO Formulation}

This chapter discusses the detailed rigorous formulation for the DRTO formulation. It starts with the description of the dynamic models used in the formulation and then moves on to the MPC formulation and the optimization formulation that describes the general control structures. The strategies for implementation and solutions are subsequently discussed and the efficacy of such a DRTO formulation is illustrated by both linear and nonlinear case studies.

The formulations and results in this chapter have been submitted to and presented in:

Li, H. and Swartz, C. L. (2017), "Coordination of distributed mpc systems through dynamic real-time optimization with closed-loop prediction", Computer Aided Chemical Engineering, Vol. 40, pp. 1603 1608. Elsevier.

Li, H. and Swartz, C. L. (2017), "Dynamic Real-Time Optimization of Distributed MPC Systems Using Rigorous Closed-Loop Prediction", Submitted to Computers and Chemical Engineering, in review.

Li, H. and Swartz, C. L. (2017), "Dynamic Real-Time Optimization of Distributed 
MPC Systems Using Rigorous Closed-Loop Prediction", AICHE Annual Meeting (2017), Minneapolis, MN, USA.

\subsection{Closed-loop DRTO (CL-DRTO) formulation for distributed MPC systems}

The conceptual design of the two-layer architecture for coordination of distributed MPCs is shown in Fig. 3.1. The upper layer incorporates a full plant model as a surrogate to generate the predicted plant response. The MPC optimization subproblems are embedded along the DRTO prediction horizon and generate the control actions for the DRTO plant model whose outputs provide disturbance estimates for subsequent MPC calculations. This sequential closed-loop prediction is optimized under a specified DRTO objective function (typically economics-based) and generates set-point trajectories for the lower level distributed MPCs that perform subsequent control tracking. The variables appearing in Fig. 3.1 are explained in detail in subsequent sections.

\subsubsection{Preliminaries: Full-scale and local plant models}

For the purpose of this study, we assume that the models available for the DRTO formulation are linear for both the full-scale plant model and local plant models. The disturbance estimate corrects the offsets generated by plant-model mismatch. However, the DRTO formulation is capable of handling a nonlinear model of the plant in the primary optimization problem as shown in Jamaludin and Swartz 2016 for application to centralized MPC systems. It can potentially incorporate nonlinear MPCs in the formulation as well, which is addressed in Chapter 5. 


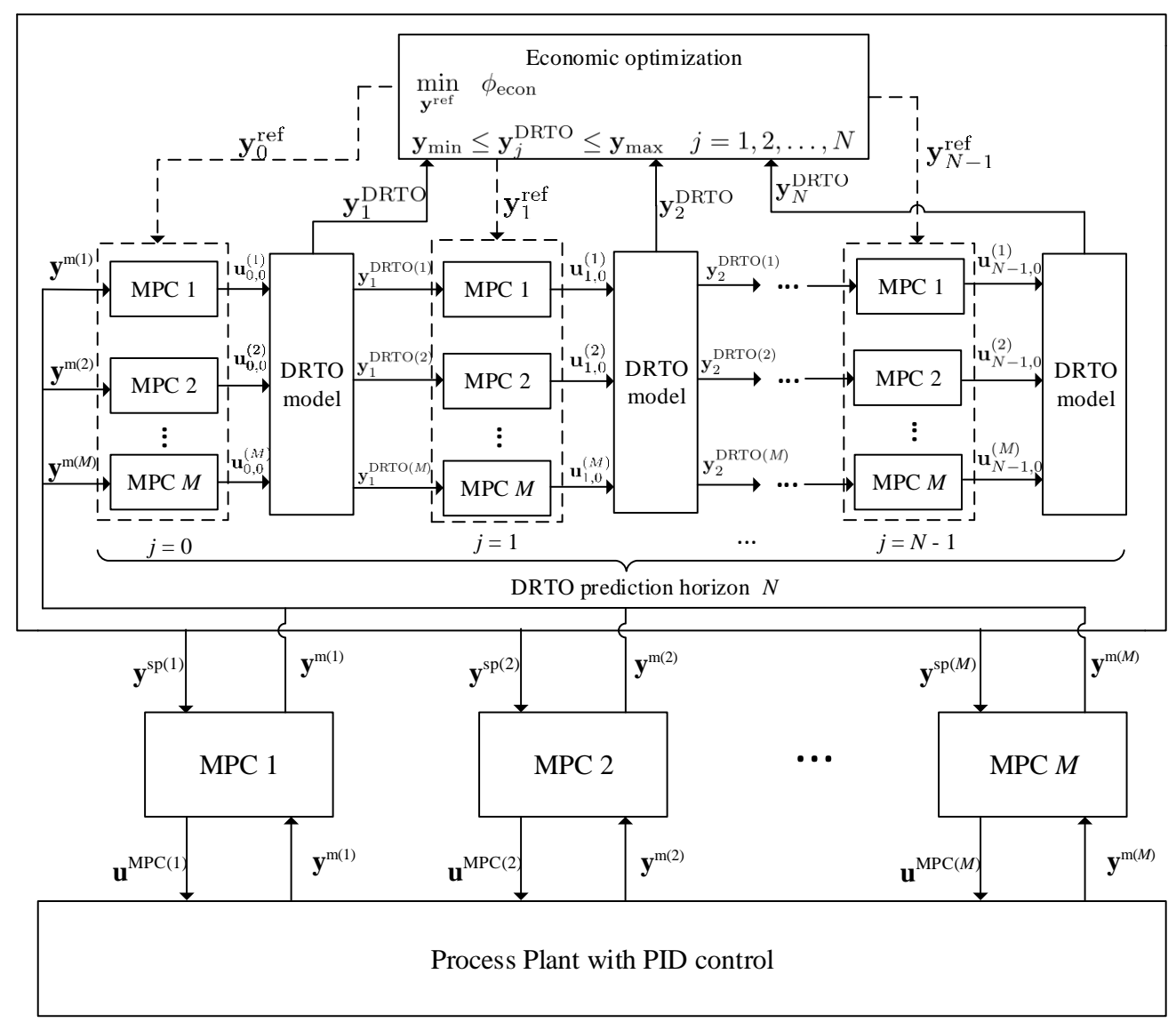

Figure 3.1: The control architecture for coordination of distributed MPCs with CL-DRTO approach. 
Consider the linear discrete-time full-scale plant model available for the DRTO layer to generate the plant response at each step in the DRTO prediction horizon $j=$ $1, \ldots, N$ :

$$
\begin{aligned}
& \mathbf{x}_{j+1}^{\mathrm{DRTO}}=A \mathbf{x}_{j}^{\mathrm{DRTO}}+B \mathbf{u}_{j}^{\mathrm{DRTO}} \\
& \mathbf{y}_{j}^{\mathrm{DRTO}}=C \mathbf{x}_{j}^{\mathrm{DRTO}}
\end{aligned}
$$

where $\mathbf{x}_{j}^{\text {DRTO }} \in R^{n_{x}}, \mathbf{u}_{j}^{\text {DRTO }} \in R^{n_{u}}$ and $\mathbf{y}_{j}^{\text {DRTO }} \in R^{n_{y}}$ are the states, inputs and outputs, respectively, of the DRTO plant model at time increment $j$.

We consider the full plant model to be decomposed into $M$ subsystems. The local plant model available for the MPC optimization subproblems of each subsystem $i$ at the DRTO prediction horizon step $j$ can be written as:

$$
\begin{aligned}
\mathbf{x}_{j, k+1}^{(i)} & =A_{i i} \mathbf{x}_{j, k}^{(i)}+B_{i i} \mathbf{u}_{j, k}^{(i)} \\
\mathbf{y}_{j, k}^{(i)} & =C_{i i} \mathbf{x}_{j, k}^{(i)}
\end{aligned}
$$

where $\mathbf{x}^{(i)} \in R^{n_{x}^{(i)}}, \mathbf{u}^{(i)} \in R^{n_{u}^{(i)}}$ and $\mathbf{y}^{(i)} \in R^{n_{y}^{(i)}}$ are vectors of states, inputs and outputs, respectively corresponding to the $i$ th subsystem, and $A_{i i}, B_{i i}$, and $C_{i i}$ are submatrices of the full plant matrices $A, B$, and $C$. We note that the MPC submodels do not include off-diagonal submatrices, thus the states in general evolve differently than those of the DRTO plant model.

\subsubsection{MPC formulation}

The MPC formulation employed in the subsequent sections corresponds to Quadratic Dynamic Matrix Control (QDMC) Garcia and Morshedi, 1986; Maciejowski, 2002. The model embedded in the formulation adopts a state-space formulation, with constrained inputs. Since multiple distributed MPCs will be taken into consideration, 
the MPC formulation for subsystem $i$ at DRTO prediction step $j$ takes the form,

$$
\begin{aligned}
\min _{\mathbf{u}_{j, k}^{(i)}} \phi_{j}^{(i)} & =\sum_{k=1}^{p}\left(\mathbf{y}_{j, k}^{(i)}-\mathbf{y}_{j, k}^{\mathrm{sp}(i)}\right)^{T} Q^{(i)}\left(\mathbf{y}_{j, k}^{(i)}-\mathbf{y}_{j, k}^{\mathrm{sp}(i)}\right) \\
& +\sum_{k=0}^{m-1}\left(\boldsymbol{\Delta} \mathbf{u}_{j, k}^{(i)}\right)^{T} R^{(i)}\left(\Delta \mathbf{u}_{j, k}^{(i)}\right)+\sum_{k=0}^{m-1}\left(\mathbf{u}_{j, k}^{(i)}-\mathbf{u}_{j, k}^{\mathrm{sp}(i)}\right)^{T} S^{(i)}\left(\mathbf{u}_{j, k}^{(i)}-\mathbf{u}_{j, k}^{\mathrm{sp}(i)}\right) \\
\text { s.t. } \quad \mathbf{x}_{j, k+1}^{(i)} & =A_{i i} \mathbf{x}_{j, k}^{(i)}+B_{i i} \mathbf{u}_{j, k}^{(i)}, \quad k=0, \ldots, m-1 \\
\mathbf{x}_{j, k+1}^{(i)} & =A_{i i} \mathbf{x}_{j, k}^{(i)}+B_{i i} \mathbf{u}_{j, m-1}^{(i)}, \quad k=m, \ldots, p-1 \\
\mathbf{y}_{j, k}^{(i)} & =C_{i i} \mathbf{x}_{j, k}^{(i)}+\mathbf{d}_{j, k}^{(i)}, \quad k=1, \ldots, p \\
\Delta \mathbf{u}_{j, k}^{(i)} & =\mathbf{u}_{j, k}^{(i)}-\mathbf{u}_{j, k-1}^{(i)}, \quad k=0, \ldots, m-1 \\
\mathbf{u}_{\min }^{(i)} & \leq \mathbf{u}_{j, k}^{(i)} \leq \mathbf{u}_{\max }^{(i)}, \quad k=0, \ldots, m-1
\end{aligned}
$$

where $\phi_{j}^{(i)}$ is a quadratic MPC objective function, and $p$ and $m$ are prediction and control move horizons, respectively. $\boldsymbol{\Delta} \mathbf{u}_{j, k}^{(i)}$ denotes the manipulated input changes, and $\mathbf{u}_{\min }^{(i)}$ and $\mathbf{u}_{\max }^{(i)}$ are the input constraint bounds. $\mathbf{y}_{j, k}^{\mathrm{sp}(i)}$ and $\mathbf{u}_{j, k}^{\mathrm{sp}(i)}$ represent the set-point trajectories for outputs and inputs, respectively. $Q^{(i)}$ and $S^{(i)}$ are set-point tracking weighting matrices, and the $R^{(i)}$ are positive semi-definite move suppression weighting matrices.

The disturbance estimate is computed as the difference between the measured output at current time point and the predicted output based on prior information, and is kept constant over the prediction horizon Cutler and Ramaker, 1979; Garcia and Morshedi, 1986]. Thus the disturbance for the MPC problem corresponding to DRTO time step $j$ for subsystem $i$ is calculated as:

$$
\mathbf{d}_{j, k}^{(i)}=\mathbf{y}_{j}^{\mathrm{m}(i)}-C_{i i} \mathbf{x}_{j, 0}^{(i)}, \quad k=1, \ldots, p
$$

where $\mathbf{y}_{j}^{\mathrm{m}(i)}$ represents the measured output corresponding to subsystem $i$ at DRTO 
time step $j$, and $\mathbf{x}_{j, 0}^{(i)}$ is the initial state estimate obtained, for example, by applying the dynamic model to advance the state from the previous time step as $\mathbf{x}_{j, 0}^{(i)}=A_{i i} \mathbf{x}_{j-1,0}^{(i)}+$ $B_{i i} \mathbf{u}_{j-1,0}^{(i)}$. In the DRTO formulation, the output of the full plant model, $\mathbf{y}_{j}^{\mathrm{DRTO}(i)}$, is used as a surrogate measurement for DRTO prediction steps $j=1, \ldots, N$. For $j=0$ (corresponding to the execution of the DRTO calculation) the actual plant measurement is used. We also note that according to 3.5

$$
\Delta \mathbf{u}_{j, 0}^{(i)}=\mathbf{u}_{j, 0}^{(i)}-\mathbf{u}_{j,-1}^{(i)}
$$

Here, $\mathbf{u}_{j,-1}^{(i)}$ corresponds to the first control move calculated at the previous time step (and applied to the DRTO model as the control input), i.e.

$$
\mathbf{u}_{j,-1}^{(i)}=\mathbf{u}_{j-1,0}^{(i)}, \quad j=1, \ldots, N
$$

For $j=0, \mathbf{u}_{j,-1}^{(i)}$ is taken as the most recent input applied to the actual plant.

We assume that the process is open-loop stable (or that it has been stabilized via feedback control), and that a sufficiently long prediction horizon is chosen such that closed-loop stability is maintained over the range of operating conditions considered. This is a common assumption in industrial MPC applications. However, alternative MPC formulations that include, for example, a terminal penalty and/or terminal constraint for which stability can be guaranteed under certain conditions, can in principle be accommodated by the proposed formulation. This is a topic for future study. 


\subsubsection{Optimization formulation}

The two dynamic models for the primary optimization problem and MPC optimization subproblems are embedded in the DRTO formulation as 3.9 ,

$$
\begin{aligned}
& \min _{\mathbf{y}^{\text {ref }}, \mathbf{u}^{\text {ref }}} \phi^{\text {DRTO }}\left(\mathbf{x}^{\text {DRTO }}, \mathbf{y}^{\text {DRTO }}, \mathbf{u}^{\text {DRTO }}\right) \\
& \text { s.t. } \mathbf{x}_{j+1}^{\mathrm{DRTO}}=A \mathbf{x}_{j}^{\mathrm{DRTO}}+B \mathbf{u}_{j}^{\mathrm{DRTO}}, \quad j=0, \ldots, N-1 \\
& \mathbf{y}_{j}^{\mathrm{DRTO}}=C \mathbf{x}_{j}^{\mathrm{DRTO}}+\mathbf{d}_{j}^{\mathrm{DRTO}}, \quad j=1, \ldots, N \\
& \mathrm{~g}^{\mathrm{DRTO}}\left(\mathbf{x}^{\mathrm{DRTO}}, \mathbf{y}^{\mathrm{DRTO}}\right) \geq \mathbf{0} \\
& \mathbf{h}_{i}^{\mathrm{ref}}\left(\mathbf{y}^{\mathrm{ref}}, \mathbf{u}^{\mathrm{ref}}, \mathbf{y}^{\mathrm{sp}(i)}, \mathbf{u}^{\mathrm{sp}(i)}\right)=\mathbf{0}, \quad i=1, \ldots, M \\
& \mathbf{g}^{\mathrm{ref}}\left(\mathbf{y}^{\mathrm{ref}}, \mathbf{u}^{\mathrm{ref}}\right) \geq \mathbf{0} \\
& \mathbf{u}_{j}^{\mathrm{DRTO}}=\left[\mathbf{u}_{j, 0}^{(1) T}, \ldots, \mathbf{u}_{j, 0}^{(M) T}\right]^{T}, \quad j=0, \ldots, N-1, \\
& \mathbf{d}_{j}^{\text {DRTO }}=\mathbf{y}^{\mathrm{m}}-C \mathbf{x}_{0}^{\text {DRTO }}, \quad j=1, \ldots, N \\
& \mathbf{u}_{j, 0}^{(i)} \in \arg \min _{\mathbf{u}_{j, k}^{(i)}} \quad \phi_{j}^{(i)}=\sum_{k=1}^{p}\left(\mathbf{y}_{j, k}^{(i)}-\mathbf{y}_{j, k}^{\mathrm{sp}(i)}\right)^{T} Q^{(i)}\left(\mathbf{y}_{j, k}^{(i)}-\mathbf{y}_{j, k}^{\mathrm{sp}(i)}\right) \\
& +\sum_{k=0}^{m-1}\left(\boldsymbol{\Delta} \mathbf{u}_{j, k}^{(i)}\right)^{T} R^{(i)}\left(\boldsymbol{\Delta} \mathbf{u}_{j, k}^{(i)}\right)+\sum_{k=0}^{m-1}\left(\mathbf{u}_{j, k}^{(i)}-\mathbf{u}_{j, k}^{\mathrm{sp}(i)}\right)^{T} S^{(i)}\left(\mathbf{u}_{j, k}^{(i)}-\mathbf{u}_{j, k}^{\mathrm{sp}(i)}\right) \\
& \text { s.t. } \quad \mathbf{x}_{j, k+1}^{(i)}=A_{i i} \mathbf{x}_{j, k}^{(i)}+B_{i i} \mathbf{u}_{j, k}^{(i)}, \quad k=0, \ldots, m-1 \\
& \mathbf{x}_{j, k+1}^{(i)}=A_{i i} \mathbf{x}_{j, k}^{(i)}+B_{i i} \mathbf{u}_{j, m-1}^{(i)}, \quad k=m, \ldots, p-1 \\
& \mathbf{y}_{j, k}^{(i)}=C_{i i} \mathbf{x}_{j, k}^{(i)}+\mathbf{d}_{j, k}^{(i)}, \quad k=1, \ldots, p \\
& \mathbf{d}_{j, k}^{(i)}=\mathbf{y}^{\mathrm{m}(i)}-C_{i i} \mathbf{x}_{j, 0}^{(i)}, \quad k=1, \ldots, p, \quad j=0 \\
& \mathbf{d}_{j, k}^{(i)}=\mathbf{y}_{j}^{\operatorname{DRTO}(i)}-C_{i i} \mathbf{x}_{j, 0}^{(i)}, \quad k=1, \ldots, p, \quad j>0 \\
& \Delta \mathbf{u}_{j, k}^{(i)}=\mathbf{u}_{j, k}^{(i)}-\mathbf{u}_{j, k-1}^{(i)}, \quad k=0, \ldots, m-1 \\
& \mathbf{u}_{\min }^{(i)} \leq \mathbf{u}_{j, k}^{(i)} \leq \mathbf{u}_{\max }^{(i)}, \quad k=0, \ldots, m-1
\end{aligned}
$$

where $\phi^{\text {DRTO }}$ represents a DRTO cost function, and $\mathbf{x}^{\text {DRTO }}, \mathbf{u}^{\text {DRTO }}$ and $\mathbf{y}^{\text {DRTO }}$ are composite vectors of the DRTO states, inputs and outputs $\left(\mathbf{x}_{j}^{\text {DRTO }}, \mathbf{u}_{j}^{\text {DRTO }}, \mathbf{y}_{j}^{\text {DRTO }}\right)$ over all time increments $j$ over the DRTO horizon. $\mathbf{y}^{\text {ref }}$ and $\mathbf{u}^{\text {ref }}$ are composite refer- 
ence trajectories for all plant outputs and inputs respectively over the DRTO prediction horizon $N$, which constitute the optimization degrees of freedom in the primary optimization problem, from which set-point trajectories for the MPC subproblems along the the DRTO prediction horizon are extracted. The mapping between the reference and set-point trajectories is expressed through constraints $\mathbf{h}_{i}^{\text {ref }}$, and is described in more detail in the following. Consider $\mathbf{y}_{j}^{\text {ref }}$ and $\mathbf{u}_{j}^{\text {ref }}$ as the output and input reference trajectory values corresponding to DRTO time step $j$, and $\mathbf{y}_{j, k}^{\mathrm{sp}(i)}$ and $\mathbf{u}_{j, k}^{\mathrm{sp}(i)}$ as the set-point trajectories for the outputs and inputs for the MPC subproblem at DRTO step $j$ for MPC subsystem $i$ at step $k$ in the MPC prediction horizon. The extraction of the set-point trajectory for MPC subproblem $j$ and subsystem $i$ from the reference trajectory can then be represented as

$$
\begin{aligned}
& \mathbf{y}_{j, k}^{\mathrm{sp}(i)}=E_{y}^{(i)} \mathbf{y}_{j+k}^{\mathrm{ref}}, \quad k=1, \ldots, p \\
& \mathbf{u}_{j, k}^{\mathrm{sp}(i)}=E_{u}^{(i)} \mathbf{u}_{j+k}^{\mathrm{ref}}, \quad k=0, \ldots, m-1
\end{aligned}
$$

where $E_{y}^{(i)} \in R^{n_{y}^{(i)} \times n_{y}}$ and $E_{u}^{(i)} \in R^{n_{u}^{(i)} \times n_{u}}$ are matrices that map the full plant outputs and inputs to the subsystem outputs and inputs. For example, for a system with three outputs that is partitioned into two subsystems with the first containing $y_{1}$ and the second containing $y_{2}$ and $y_{3}$, the $E_{y}^{(i)}$ matrices would be

$$
E_{y}^{(1)}=\left[\begin{array}{lll}
1 & 0 & 0
\end{array}\right], \quad E_{y}^{(2)}=\left[\begin{array}{lll}
0 & 1 & 0 \\
0 & 0 & 1
\end{array}\right]
$$

Fig. 3.2 illustrates the extraction of the MPC set-point trajectory from the composite reference trajectory for a centralized MPC configuration. We remark that the MPC subproblems toward the end of the DRTO horizon require reference values that extend beyond $N$. In this situation, reference trajectory values beyond $N$ are set equal to the values at $N \cdot \mathbf{g}^{\text {DRTO }}$ defines output and state constraints, while $\mathbf{g}^{\text {ref }}$ denotes the constraints for reference trajectories which can be slightly less stringent than the 


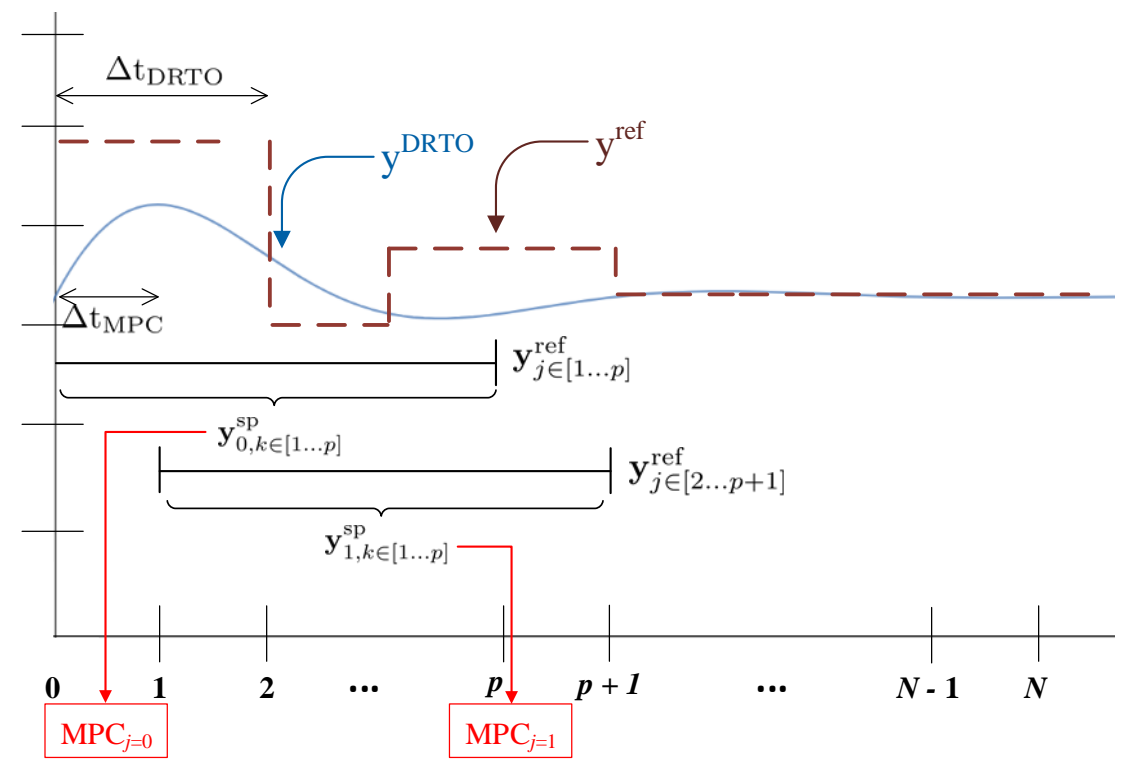

Figure 3.2: The illustration of set-point extraction from reference trajectory.

output constraints.

The first sets of control actions $\mathbf{u}_{j, 0}^{(i)}$ calculated by the MPC optimization subproblem of each distributed MPC at the current time step are equated to the inputs of primary optimization problem $\mathbf{u}_{j}^{\mathrm{DRTO}}$ at that time instance via

$$
\mathbf{u}_{j}^{\text {DRTO }}=\left[\mathbf{u}_{j, 0}^{(1) T}, \ldots, \mathbf{u}_{j, 0}^{(M) T}\right]^{T}, j=0, \ldots, N-1
$$

where $\mathbf{u}_{j}^{\text {DRTO }}$ is applied to the full plant model, hence comprises the inputs corresponding to all the plant subsystems.

A disturbance estimate for the primary optimization problem is computed as the difference between the outputs measured at current instance immediately before the DRTO execution and predicted output values based on the prediction made at the previous time instance, following a similar approach to that described earlier for the MPC subproblems. The formulation readily admits alternative disturbance handling, state estimation and MPC formulation schemes. We note that the input tracking 
term in the MPC objective function can be especially useful to eliminate degrees of freedom for a non-square system, where the set-points generated for inputs correspond to so-called ideal resting values Qin and Badgwell, 2003. We remark finally that inclusion of input constraints in the MPC subproblems removes the requirement for their inclusion in the primary DRTO problem, since the subproblems form part of the overall DRTO optimization problem; inclusion of input constraints in the primary DRTO problem is therefore redundant. In our implementation, we apply output constraints at the primary DRTO level rather than in the MPC subproblems, as the latter is known to contribute to problems of infeasibility and/or instability Zafiriou and Marchal, 1991.

Following the execution of the DRTO optimization problem $(3.9)$, the optimal setpoint trajectories are supplied to the lower-level plant MPCs as

$$
\mathbf{y}^{\mathrm{sp}(i)}=\left[\mathbf{y}_{j, 1}^{\mathrm{sp}(i) T}, \ldots, \mathbf{y}_{j, p}^{\mathrm{sp}(i) T}\right]^{T}, \quad i=1, \ldots, M
$$

with $j=0$ initially, and incremented for each successive MPC execution until the next DRTO execution, at which time the index $j$ is reset to 0 and the process repeated. The implementation scheme is illustrated in Fig. 3.1, with the time-shifted set-point trajectories required for successive MPC executions illustrated in Fig. 3.2. We remark that it is only the set-point trajectories that are passed from the DRTO system to the plant MPCs, and not the inputs. The plant inputs are calculated by the local plant MPCs, in accordance with the set-point trajectories.

\subsubsection{Solution approach}

The proposed formulation can be solved in principle by adopting a sequential solution approach, in which a closed-loop simulation is carried out for iterates of specified output and input reference trajectories. However, to avoid potential difficulties due 
to nonsmoothness induced by the MPC input constraints, we follow instead a simultaneous solution approach in which the MPC QP subproblems are replaced by their equivalent first-order Karush-Kuhn-Tucker (KKT) optimality conditions resulting in a single-level mathematical program with complementarity constraints (MPCC) Baker and Swartz, 2008; Jamaludin and Swartz, 2017b. This transformation can be illustrated using the general QP form:

$$
\begin{array}{ll}
\min _{z} & \frac{1}{2} \mathbf{z}^{T} H \mathbf{z}+\mathbf{g}^{T} \mathbf{z} \\
\text { s.t. } & A \mathbf{z}=\mathbf{b}, \\
& \mathbf{z} \geq \mathbf{0}
\end{array}
$$

whose KKT optimality conditions are:

$$
\begin{aligned}
& H \mathbf{z}+\mathbf{g}-A^{T} \boldsymbol{\nu}-\boldsymbol{\eta}=\mathbf{0} \\
& A \mathbf{z}-\mathbf{b}=\mathbf{0}, \\
& z_{i} \eta_{i}=0, \quad i \in I \\
& (\mathbf{z}, \boldsymbol{\eta}) \geq \mathbf{0}
\end{aligned}
$$

The set of constraints derived from MPC optimization subproblems contains complementarity constraints that may pose difficulties to nonlinear programming (NLP) algorithms. Here, this is addressed through an exact penalty formulation in which the complementarity constraints are removed and incorporated as a suitably weighted penalty term in the objective function Ralph and Wright, 2004 Jamaludin and Swartz, 2017b. 


\subsubsection{Implementation variants}

The proposed DRTO formulation can be used in two ways: economic dynamic optimization based on an economic objective function, and target tracking which adopts a least-squares form when process target values are known a priori.

\section{Economic objective function}

A profit based objective function can typically be written as,

$$
\phi=\Delta t_{\mathrm{MPC}} \sum_{j=1}^{N} \varphi_{j}\left(\mathbf{x}_{j}^{\mathrm{DRTO}}, \mathbf{y}_{j}^{\mathrm{DRTO}}, \mathbf{u}_{j-1}^{\mathrm{DRTO}}\right)
$$

where $\varphi_{j}$ represents profit per unit time. A key application of DRTO is to plants undergoing frequent product transitions, in which case it is important to capture the characteristic that revenue accrues only when the product quality is within a prescribed specification band. We incorporate this by following the approach in Jamaludin and Swartz 2017b in which the revenue term is multiplied by $R^{1} R^{2}$, where $R^{1}$ and $R^{2}$ are hyperbolic tangent approximations of switching functions defined as

$$
\begin{aligned}
& R^{1}=\frac{1}{2} \tanh \left[\gamma\left(y-y^{\text {target }}+\delta y^{\text {target }}\right)\right]+\frac{1}{2} \approx\left\{\begin{array}{l}
0, y<y-\delta y^{\text {target }} \\
1, y>y-\delta y^{\text {target }}
\end{array}\right. \\
& R^{2}=\frac{1}{2} \tanh \left[\gamma\left(y^{\text {target }}+\delta y^{\text {target }}-y\right)\right]+\frac{1}{2} \approx\left\{\begin{array}{l}
0, y>y+\delta y^{\text {target }} \\
1, y<y+\delta y^{\text {target }}
\end{array}\right.
\end{aligned}
$$

where $\gamma$ is used to defined the steepness of this product transition function. Thus, $R^{1} R^{2} \approx 1$ if the product quality represented by the output $y$ falls within specification bounds $\left[y-\delta y^{\text {target }}, y+\delta y^{\text {target }}\right]$, and $\approx 0$ otherwise. 


\section{Target tracking}

The DRTO strategy is also an effective mechanism to drive the process outputs and/or inputs to specified target values through the prescription of set-point trajectories to the distributed MPCs. In this way, the final targets are not initially fixed as set-point trajectories along the horizon. The DRTO strategy is able to predict the future interactions between controllers and the process and judiciously minimize the differences between the response and the target.

In this study, the target tracking functionality is addressed in a weighted least squares fashion, with the objective function adopting the following form:

$$
\phi=\Delta t_{\mathrm{MPC}} \sum_{j=1}^{N}\left(\sum_{k \in K} w_{k}\left(y_{j, k}^{\mathrm{DRTO}}-y_{k}^{\mathrm{target}}\right)^{2}+\sum_{i \in I} w_{i}\left(u_{j-1, i}^{\mathrm{DRTO}}-u_{i}^{\mathrm{target}}\right)^{2}\right)
$$

where $K$ and $I$ represent the set of outputs and inputs respectively for which target

values are specified, $y_{j, i}^{\text {DRTO }}$ and $u_{j, i}^{\text {DRTO }}$ represent the $i$ th component of $\mathbf{y}_{j}^{\text {DRTO }}$ and $\mathbf{u}_{j}^{\text {DRTO }}$ respectively, and the $w_{i}$ are weights.

\section{Set-point variation reduction}

The DRTO solution may exhibit unnecessarily high variation in the reference trajectories due to solution nonuniqueness or a relatively flat objective function surface. An effective mechanism to mitigate this effect is to maintain the reference trajectory constant over a specified number of discretization intervals. We consider the effect of this set-point hold (SPH) strategy in the next section. 


\subsection{Case Studies}

\subsubsection{Case Study 1: Linear Dynamic System}

\section{Base case}

This case study explores effect of centralized, decentralized and distributed MPC configurations for a linear dynamic system when targets of the outputs are known. Consider the following transfer function model that involves two inputs and two outputs, taken from Chien et al. [1999]:

$$
G(s)=\left[\begin{array}{ll}
\frac{22.89}{4.572 s+1} e^{-0.2 s} & \frac{-11.64}{1.807 s+1} e^{-0.4 s} \\
\frac{4.689}{2.174 s+1} e^{-0.2 s} & \frac{5.80}{1.801 s+1} e^{-0.4 s}
\end{array}\right]
$$

This transfer function matrix in 3.20 is partitioned into two subsystems shown in (3.21), each of which consists of a single transfer function extracted from the diagonal components of the full matrix, such that the subsystems do not acknowledge the interactions between them. However, the full transfer function is used in the DRTO level to generate the plant response.

$$
\begin{aligned}
G_{1}(s) & =\frac{22.89}{4.572 s+1} e^{-0.2 s} \\
G_{2}(s) & =\frac{5.80}{1.801 s+1} e^{-0.4 s}
\end{aligned}
$$

The objective function for the target tracking is defined as,

$$
\phi=\Delta t_{\mathrm{MPC}} \sum_{j=1}^{N}\left(y_{j, 1}^{\mathrm{DRTO}}-1\right)^{2}+\left(y_{j, 2}^{\mathrm{DRTO}}-2\right)^{2}
$$


Table 3.1: Design parameters for the DRTO and MPC layers in Case Study 1.

\begin{tabular}{llll}
\hline Parameter & Description & $\begin{array}{l}\text { Value } \\
\text { ized MPC) }\end{array}$ & $\begin{array}{l}\text { (central- } \\
\text { decentralized MPCs) }\end{array}$ \\
\hline$\Delta t_{M P C}$ & MPC sample time & $1 \mathrm{hr}$ & $1 \mathrm{hr}$ \\
$\Delta t_{D R T O}$ & DRTO sample time & - & $1 \mathrm{hr}$ \\
$p$ & prediction horizon & 10 & 10 \\
$m$ & control horizon & 5 & 5 \\
$Q$ & output tracking weight & $2 \mathrm{I}$ & 2 \\
$R$ & move suppression weight & $20 \mathrm{I}$ & 20 \\
$N$ & optimization horizon & - & 50 \\
$\rho$ & complementarity penalty & - & 100 \\
& parameter & & \\
\hline
\end{tabular}

subject to constraints,

$$
\begin{aligned}
& 0 \leq y_{j, 1}^{\mathrm{DRTO}} \leq 2, \quad 0 \leq y_{j, 2}^{\mathrm{DRTO}} \leq 4, \quad j=1, \ldots, N \\
& 0 \leq y_{j, 1}^{\mathrm{ref}} \leq 2, \quad j=0, \ldots, N-1 \\
& 0 \leq y_{j, 2}^{\mathrm{ref}} \leq 4, \quad j=0, \ldots, N-1 \\
& -5 \leq u_{j, k}^{(1)}, u_{j, k}^{(2)} \leq 5, \quad j=0, \ldots, N-1, \quad k=0, \ldots, m-1
\end{aligned}
$$

where $y_{j, i}^{\mathrm{DRTO}}$ and $y_{j, i}^{\mathrm{ref}}$ are the $i$ th component of $\mathbf{y}_{j}^{\mathrm{DRTO}}$ and $\mathbf{y}_{j}^{\mathrm{ref}}$ respectively. The target for the first and second outputs are set as 1 and 2, respectively. The set-point trajectories for both centralized and decentralized MPCs are kept constant at the target values. The parameters for setting up the DRTO and MPC layers for both centralized and distributed cases are summarized in Table 3.1.

Fig. 3.3 shows the plant response for the centralized and decentralized MPC configurations. As shown in the figure, the decentralized case exhibits significant oscillations due to the interaction not accounted for in the MPC formulation. We observe that the response generated by decentralized MPCs exhibits inverse response for the first output $y_{1}$, since the decentralized MPCs only see the diagonal elements of the transfer 
function and there is a large negative gain in the (1,2) off-diagonal element affecting the first output. Thus, the response generated based on the control action of the first move becomes negative. Fig. 3.4 shows the response with the DRTO strategy as the supervisory layer for the distributed cases with no SPH mechanism. Rapid responses are achieved, with performance approaching that of centralized MPC. The $\mathrm{SPH}$ method is then used to reduce the set-point variation, with the set-points held constant over every 3 MPC sample intervals. The results are shown in Fig. 3.5. Due to reduced variation allowed in the trajectories by the additional constraints of setpoint hold, the performance is more sluggish compared to the counterpart without the SPH mechanism. We point out that the set-point trajectories shown in the figures are composites comprised of the portions of the set-point trajectories computed at each DRTO execution until the next (at which point an entirely new trajectory is computed). In reality, a suite of set-point trajectories is generated during the course of the simulation, with a new set of trajectories computed at each DRTO execution. A visualization of this for centralized MPC is given in Jamaludin et al. [2017]. In order to numerically evaluate the performance for each case, the sum of squared errors (SSE) between the final targets and the process responses is calculated. The results are summarized in Table 3.2. From the results in the Table 3.2, it can be concluded that the DRTO strategy is able to assign set-point trajectories to underlying MPCs flexibly based on the rigorous prediction of interactions between controllers and the process. While the centralized MPC gives the best performance, the performance of the coordinated distributed case is quite comparable. A point to note is that the limits imposed on the set-point trajectories can have a significant impact on the overall performance for the distributed case. If the upper limit for the set-points of the first output is increased to 4 instead of 2 , the distributed case outperforms the centralized counterpart due to the combined effects of the additional degrees of freedom provided by the set-point trajectories over the constant set-points used in the centralized scheme, and the relaxed set-point bounds. The average CPU time for the DRTO 

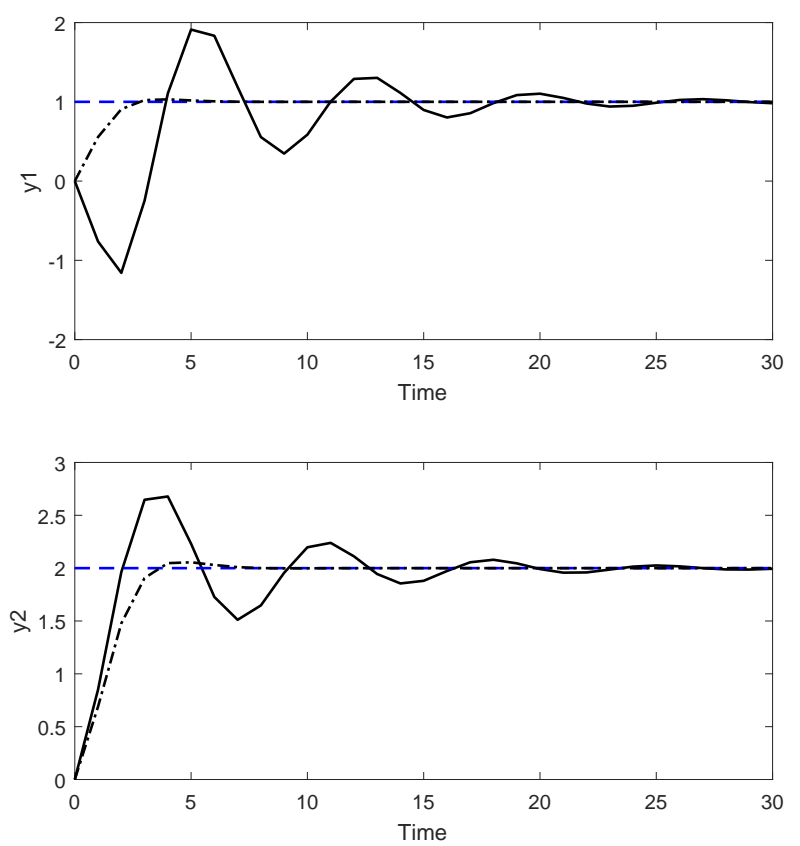

Figure 3.3: Output response under centralized and decentralized MPC alone. Dot-dashed line: centralized MPC, Solid line: decentralized MPC, Dashed line: set-point. 

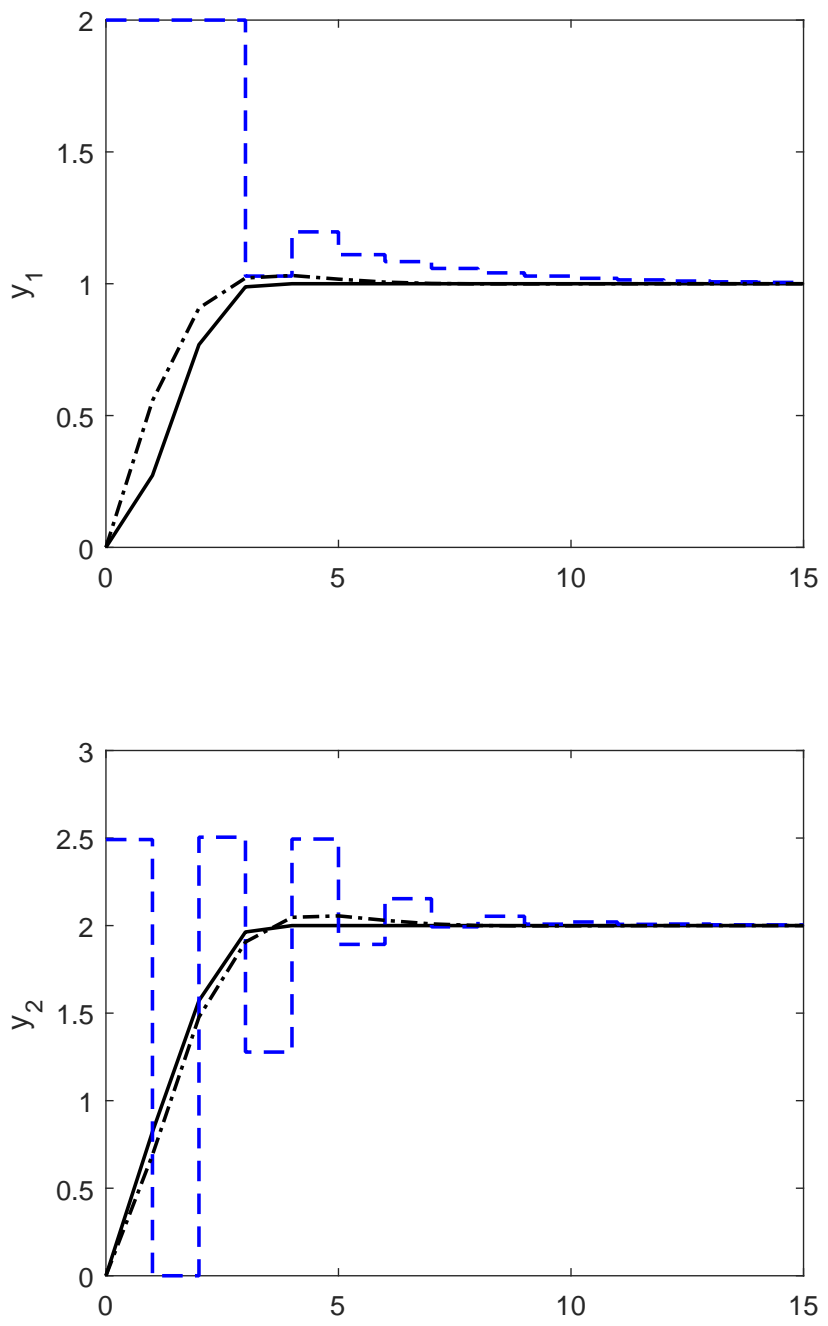

Figure 3.4: Output response (solid line) with set-points (dashed line) under distributed MPC coordinated by DRTO layer with no SPH, and output response (dot-dashed line) under centralized MPC. 
Table 3.2: Performance for configurations in Case 1 in terms of sum of squared errors (SSE).

\begin{tabular}{lllllll}
\hline Configuration Control archi- & $\begin{array}{l}\text { Reduction } \\
\text { tecture }\end{array}$ & $\mathrm{SSE}_{\mathrm{y}_{1}}$ & $\mathrm{SSE}_{\mathrm{y}_{2}}$ & $\begin{array}{l}\text { Performance Avg. } \\
\text { loss }\end{array}$ & $\begin{array}{l}\text { DRTO } \\
\text { CPU time } \\
\text { (sec) }\end{array}$ \\
\hline centralized & MPC & & & & - \\
distributed & DRTO + MPC & no SPH & 1.582 & 5.573 & $18.4 \%$ & 0.40 \\
distributed & DRTO + MPC & SPH=3 & 1.854 & 5.643 & $30.9 \%$ & 0.49 \\
decentralized & MPC & n/a & 12.881 & 6.840 & $521.5 \%$ & - \\
\hline
\end{tabular}

execution is also listed in Table 3.2, using the CONOPT solver accessed from AMPL. Given that the DRTO sample time for this case is $1 \mathrm{hr}$, the significantly lower DRTO execution time of under 1 second establishes its viability for real-time application.

\section{Impact of plant interaction effects}

In this section, a scaling factor $\alpha$ has been added to the off-diagonal elements in the original transfer function:

$$
G_{\alpha}(s)=\left[\begin{array}{ll}
\frac{22.89}{4.572 s+1} e^{-0.2 s} & \frac{-11.64 \alpha}{1.807 s+1} e^{-0.4 s} \\
\frac{4.689 \alpha}{2.174 s+1} e^{-0.2 s} & \frac{5.80}{1.801 s+1} e^{-0.4 s}
\end{array}\right]
$$

The motivation of this section is to investigate the effect of interactions among subsystems on the performance of the different control architectures. The scaling factor $\alpha$ is selected to have values of either 0 or 0.5 . When $\alpha=0$, the interaction between the subsystems is non-existent, whereas when $\alpha=0.5$, the interaction is weakened by half compared to that of the original plant considered in the previous section. The simulation procedure remains the same as in the section discussed above but for simplicity, results are only generated without the set-point variation reduction 

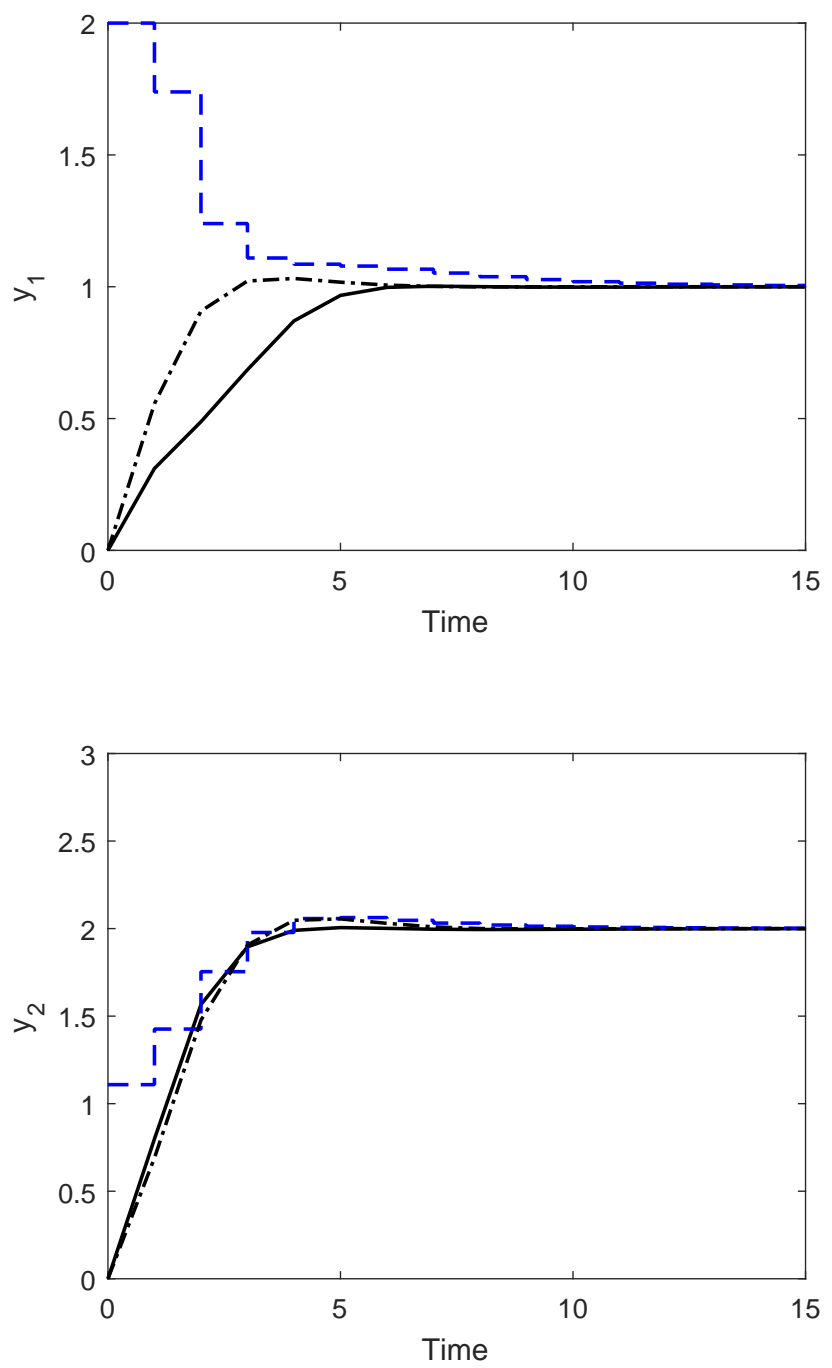

Figure 3.5: Output response (solid line) with set-points (dashed line) under distributed MPCs coordinated by DRTO layer with $\mathrm{SPH}=3$, and output response (dot-dashed line) under centralized MPC. 
Table 3.3: Performance for configurations in Case Study 1 in terms of sum of squared errors (SSE) for different interaction scaling factors $\alpha$.

\begin{tabular}{lllll}
\hline Configuration & Control architecture & SSE $_{\mathrm{y}_{1}}$ & SSE $_{\mathrm{y}_{2}}$ & $\begin{array}{l}\text { Performance loss } \\
(\alpha=1)\end{array}$ \\
\hline centralized & MPC & 1.149 & 5.626 & $0 \%$ \\
distributed & DRTO + MPC & 1.582 & 5.573 & $18.4 \%$ \\
decentralized & MPC & 12.881 & 6.840 & $521.5 \%$ \\
\hline Configuration & Control architecture & SSE $_{\mathrm{y}_{1}}$ & $\mathrm{SSE}_{\mathrm{y}_{2}}$ & $\begin{array}{l}\text { Performance loss } \\
(\alpha=0.5)\end{array}$ \\
\hline centralized & MPC & 1.207 & 6.005 & $0 \%$ \\
distributed & DRTO + MPC & 1.614 & 4.968 & $8.2 \%$ \\
decentralized & MPC & 4.234 & 5.788 & $123.6 \%$ \\
\hline Configuration & Control architecture & $\mathrm{SSE}_{\mathrm{y}_{1}}$ & $\mathrm{SSE}_{\mathrm{y}_{2}}$ & Performance loss \\
& & & & $(\alpha=0)$ \\
\hline distributed & DRTO + MPC & 1.031 & 4.826 & $0 \%$ \\
centralized & MPC & 1.233 & 6.167 & $23.7 \%$ \\
decentralized & MPC & 1.233 & 6.167 & $23.7 \%$ \\
\hline
\end{tabular}

mechanism. Table 3.3 presents the summary of performance when the scaling factor varies from 0 to 1 .

We observe from Table 3.3 that as $\alpha$ decreases, the performance of the decentralized configuration approaches that of the centralized case, since at $\alpha=0$, there is no interaction between the plant subsystems. Also, as $\alpha$ decreases, the performance of the distributed case gradually approaches that of the centralized counterpart and eventually surpasses it. This is due to a combination of the reduction in interaction effects with diminishing $\alpha$ hence improving the performance of the distributed controllers, accounting for the effects of local control action and subsystem interaction through the DRTO closed-loop prediction, and the additional degrees of freedom provided through the non-constant set-point trajectories determined in the DRTO calculation. 


\subsubsection{Case Study 2: Dynamic optimization on a nonlinear integrated plant}

This case study considers an integrated plant that consists of three units, two CSTRs and a separator, as shown in Figure 3.6. Two inlet streams $F_{10}$ and $F_{20}$ provide reactant $\mathrm{A}$ to the first and second reactor, respectively. The reactant $\mathrm{A}$ in both reactors undergoes a reaction that converts $\mathrm{A}$ into desired product $\mathrm{B}$, and small amount of product $\mathrm{B}$ is further converted to side product $\mathrm{C}$. Two reactors are configured in series and the effluent from the second reactor enters the separator which has a recirculation stream that supplies condensed overhead vapor back to the first CSTR. The purified product is withdrawn as the bottom stream. This integrated plant model is presented by Liu et al. 2009] and Farina and Scattolini 2012. The differential equations that describe the overall process can be found in Liu et al. 2009], under the assumption that the levels in the vessels are perfectly controlled. The parameters for this model is presented in Table 3.4 . The steady-state values of the model, and constraints for inputs and outputs are summarized in Tables 3.5 and 3.6, respectively. Table 3.5 also defines the partitioning of the system into two subsystems and the corresponding states, inputs and outputs.

The partial model for each distributed MPC subsystem is constructed by including only relevant equations for the subsystem. The full plant model is utilized in the DRTO formulation for generating the plant response. The partial models and full plant model are linearized at the operating point, and the discrete-time state-space forms embedded in the DRTO formulation. The nonlinear model is used to simulate the real plant response.

The temperatures of each vessel and the product concentration are selected as outputs for distributed and centralized MPCs due to monitoring of product quality and safety considerations. 


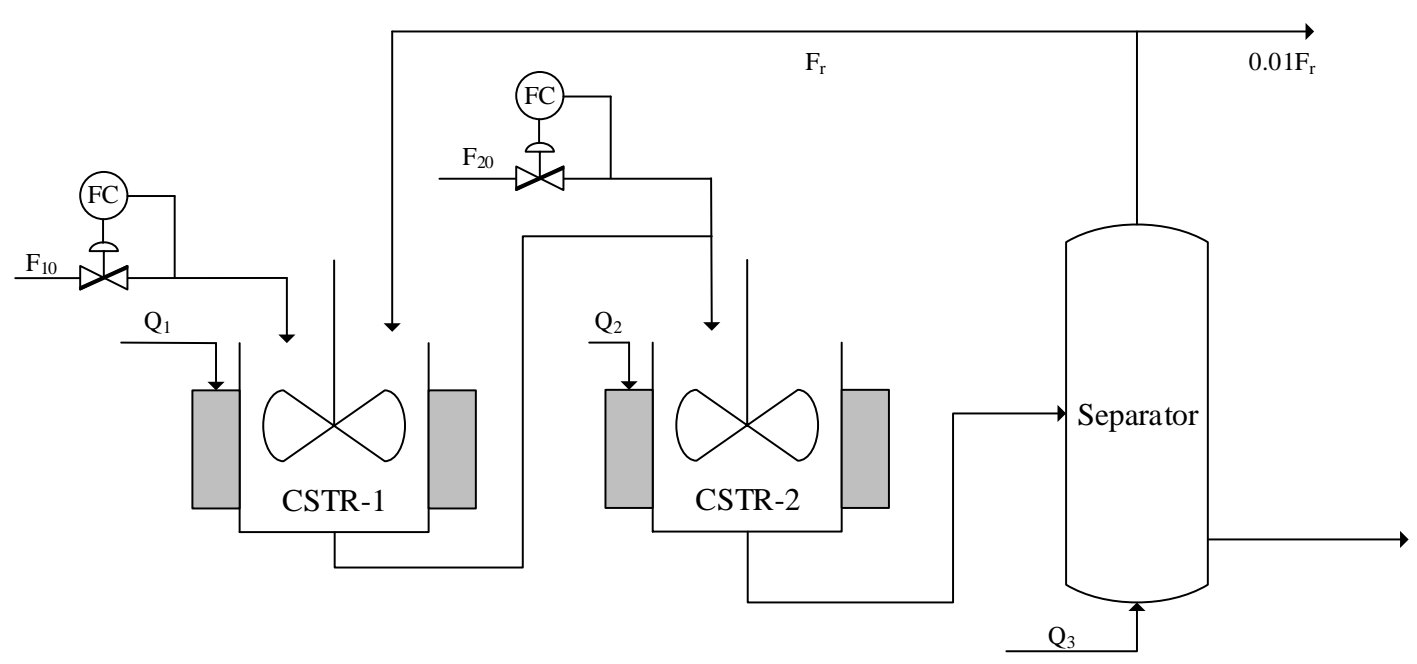

Figure 3.6: Process schematic of the integrated plant.

When this integrated plant is controlled by a centralized MPC, two degrees of freedom are available due to the non-square configuration. As a result, the inputs $Q_{1}$ and $F_{r}$ are assigned set-points along with the other four outputs.

In the distributed configuration for this system, each MPC has one degree of freedom so that one of the inputs for each MPC can be assigned a set-point trajectory. The selected inputs with set-point tracking are the heat $Q_{1}$ and the recirculation flow rate $F_{r}$.

\section{Target tracking}

When the information for specific targets required for outputs is available, the DRTO formulation can be used as a supervisory layer to oversee the transition to such targets. Compared to having constant set-points assigned directly to the centralized or distributed MPCs, the DRTO layer in the coordinated distributed configuration foresees the interaction between the controllers and plant and assigns appropriate set-point trajectories at each DRTO execution, which offers greater flexibility as well 
Table 3.4: Process parameters for the integrated plant.

\begin{tabular}{llll}
\hline \multicolumn{2}{l}{ Parameter Description } & Value & Units \\
\hline$V_{1}$ & volume of vessel 1 & 89.4 & $\mathrm{~m}^{3}$ \\
$V_{2}$ & volume of vessel 2 & 90 & $\mathrm{~m}^{3}$ \\
$V_{3}$ & volume of vessel 3 & 13.27 & $\mathrm{~m}^{3}$ \\
$k_{1}$ & first reaction constant & 0.336 & $\mathrm{~s}^{-1}$ \\
$k_{2}$ & second reaction constant & 0.089 & $\mathrm{~s}^{-1}$ \\
$E_{1}$ & activation energy for reaction 1 & -813.4 & $\mathrm{~J} / \mathrm{mol}$ \\
$E_{2}$ & activation energy for reaction 2 & -1247.1 & $\mathrm{~J} / \mathrm{mol}$ \\
$T_{10}$ & temperature of $F_{10}$ & 313 & $\mathrm{~K}$ \\
$T_{20}$ & temperature of $F_{20}$ & 313 & $\mathrm{~K}$ \\
$x_{A 10}$ & mass fraction of A in $F_{10}$ & 1 & - \\
$x_{A 20}$ & mass fraction of A in $F_{20}$ & 1 & - \\
$\Delta H_{1}$ & heat of reaction for reaction 1 & -40 & $\mathrm{~kJ} / \mathrm{kg}$ \\
$\Delta H_{2}$ & heat of reaction for reaction 2 & -50 & $\mathrm{~kJ} / \mathrm{kg}$ \\
$C_{p}$ & heat capacity & 2.5 & $\mathrm{~kJ} /(\mathrm{kg} \mathrm{K})$ \\
$\alpha_{A}$ & relative volatility of component A & 3.5 & - \\
$\alpha_{B}$ & relative volatility of component B & 0.5 & - \\
$\alpha_{C}$ & relative volatility of component C & 1.1 & - \\
$\rho$ & solution density & 0.15 & $\mathrm{~kg} / \mathrm{m}^{3}$ \\
\hline
\end{tabular}

Table 3.5: Initial steady-state values for inputs and outputs of the integrated plant.

\begin{tabular}{|c|c|c|c|c|c|}
\hline \multicolumn{6}{|c|}{ subsystem 1} \\
\hline \multicolumn{2}{|c|}{$\mathbf{x}_{1}$} & \multicolumn{2}{|c|}{$\mathbf{y}_{1}$} & \multicolumn{2}{|c|}{$\mathbf{u}_{1}$} \\
\hline variable & value & variable & value & variable & value \\
\hline$x_{A 1}$ & 0.264 & $T_{1}$ & $337.02 \mathrm{~K}$ & $F_{10}$ & $8.3 \mathrm{~m}^{3} / \mathrm{s}$ \\
\hline$x_{B 1}$ & 0.396 & & & $Q_{1}$ & $10 \mathrm{~kJ} / \mathrm{s}$ \\
\hline$T_{1}$ & $337.02 \mathrm{~K}$ & & & & \\
\hline \multicolumn{6}{|c|}{ subsystem 2} \\
\hline \multicolumn{2}{|c|}{$\mathbf{x}_{2}$} & \multicolumn{2}{|c|}{$\mathbf{y}_{2}$} & \multicolumn{2}{|c|}{$\mathbf{u}_{2}$} \\
\hline variable & value & variable & value & variable & value \\
\hline$x_{A 2}$ & 0.106 & $T_{2}$ & $344.43 \mathrm{~K}$ & $F_{20}$ & $0.5 \mathrm{~m}^{3} / \mathrm{s}$ \\
\hline$x_{B 2}$ & 0.404 & $x_{B 3}$ & $0.475 \mathrm{~K}$ & $Q_{2}$ & $10 \mathrm{~kJ} / \mathrm{s}$ \\
\hline$T_{2}$ & $344.43 \mathrm{~K}$ & $T_{3}$ & $346.51 \mathrm{~K}$ & $F_{r}$ & $4 \mathrm{~m}^{3} / \mathrm{s}$ \\
\hline$x_{A 3}$ & 0.057 & & & $Q_{3}$ & $10 \mathrm{~kJ} / \mathrm{s}$ \\
\hline$x_{B 3}$ & 0.475 & & & & \\
\hline$T_{3}$ & $346.51 \mathrm{~K}$ & & & & \\
\hline
\end{tabular}


Table 3.6: Bounds for inputs and outputs of the integrated plant.

\begin{tabular}{llllll}
\hline Variable & $\begin{array}{l}\text { lower } \\
\text { bound }\end{array}$ & $\begin{array}{l}\text { upper } \\
\text { bound }\end{array}$ & Variable & $\begin{array}{l}\text { lower } \\
\text { bound }\end{array}$ & $\begin{array}{l}\text { upper } \\
\text { bound }\end{array}$ \\
\hline$F_{10}$ & 0 & 15 & $F_{20}$ & 0 & 20 \\
$F_{r}$ & 0 & 15 & $Q_{1}$ & 0 & 50 \\
$Q_{2}$ & 0 & 100 & $Q_{3}$ & 0 & 20 \\
$T_{1}, T_{1}^{s p}$ & 326 & 357 & $T_{2}, T_{2}^{s p}$ & 324 & 364 \\
$x_{B 3}, x_{B 3}^{s p}$ & 0.45 & 0.65 & $T_{3}, T_{3}^{s p}$ & 326 & 366 \\
\hline
\end{tabular}

as interaction mitigation.

The objective function for target tracking is formed in a least-squares fashion:

$$
\begin{aligned}
\min & \Delta t_{M P C} \sum_{j=1}^{N} w_{1}\left(T_{1 j}^{\mathrm{DRTO}}-T_{1}^{\mathrm{target}}\right)^{2}+w_{2}\left(T_{2 j}^{\mathrm{DRTO}}-T_{2}^{\mathrm{target}}\right)^{2}+w_{3}\left(T_{3 j}^{\mathrm{DRTO}}-T_{3}^{\mathrm{target}}\right)^{2} \\
& +w_{4}\left(x_{B 3 j}^{\mathrm{DRTO}}-x_{B 3}^{\mathrm{target}}\right)^{2}+w_{5}\left(F_{r, j-1}^{\mathrm{DRTO}}-F_{r}^{\mathrm{target}}\right)^{2}+w_{6}\left(Q_{1, j-1}^{\mathrm{DRTO}}-Q_{1}^{\mathrm{target}}\right)^{2}
\end{aligned}
$$

where $T_{1}^{\text {target }}=347.02 \mathrm{~K}, T_{2}^{\text {target }}=354.43 \mathrm{~K}, T_{3}^{\text {target }}=347.02 \mathrm{~K}, x_{B 3}^{\text {target }}=0.475$, $F_{r}^{\text {target }}=5 \mathrm{~kg} / \mathrm{s}, Q_{1}^{\text {target }}=10.1 \mathrm{~J} / \mathrm{s}$, and weights $w_{i}=1(i, \ldots, 6)$. The decision variables in this problems are the reference trajectories, set-point trajectories, and DRTO and MPC plant states, inputs and outputs. The optimization degrees of freedom, taking into account the equality constraints, correspond to the reference trajectories for the output and input variables, $T_{1}, T_{2}, T_{3}, x_{B 3}, F_{r}$ and $Q_{1}$.

The associated design and tuning parameters are summarized in Table 3.7. The plant response for the distributed case with the DRTO formulation is shown in Fig. 3.7 for the outputs and Fig. 3.8 for the inputs. It can be seen in the graphs that the final targets assigned to the outputs and inputs are achieved. The variation in set-point trajectories helps the system act faster compared to MPC configurations alone. To make a clear comparison, the performance of centralized and decentralized 

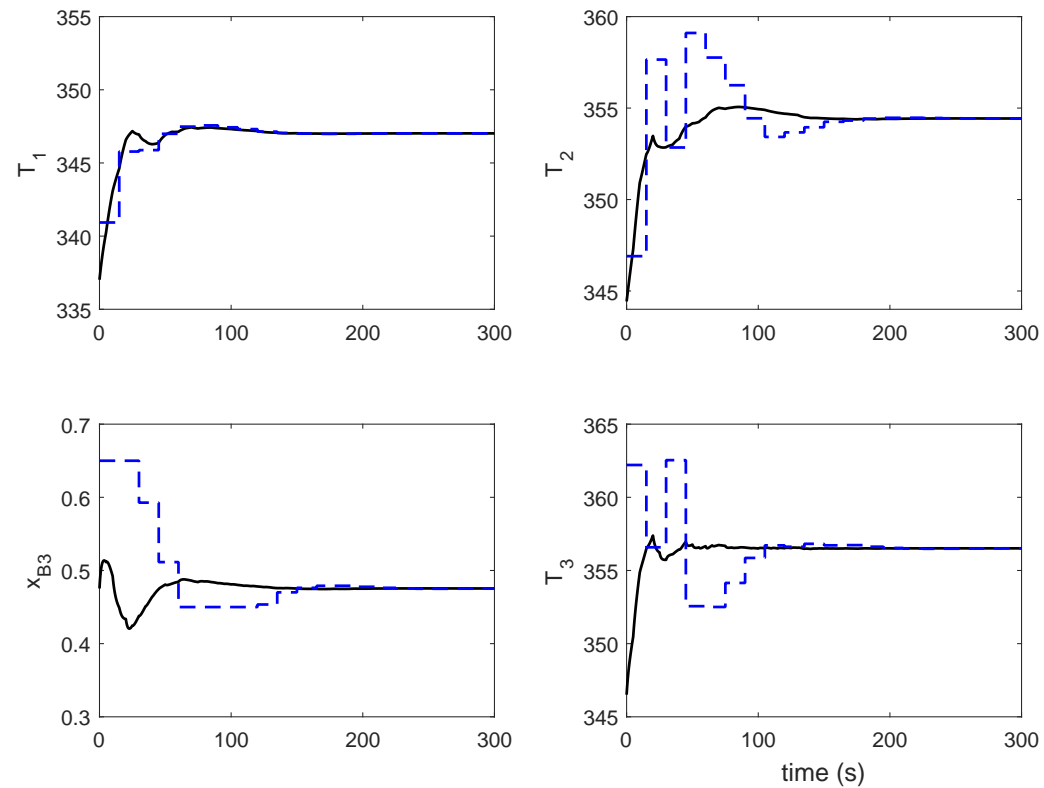

Figure 3.7: Output responses under distributed MPC with DRTO layer for target tracking case $(\mathrm{SSE}=49.8799)$.
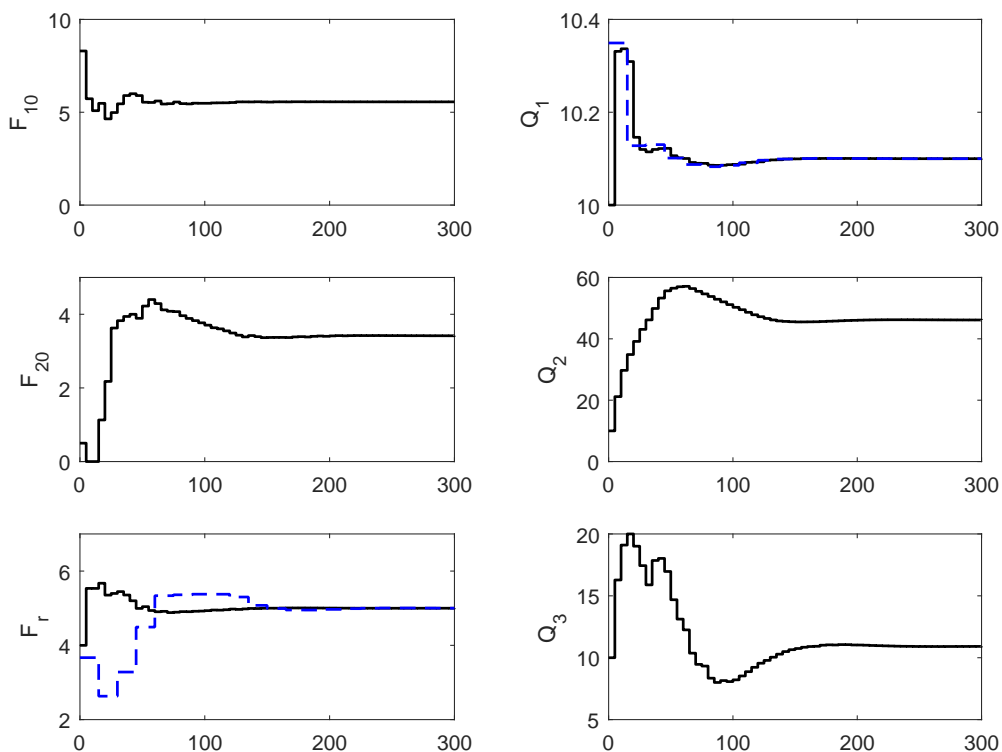

Figure 3.8: Input responses under distributed MPC with DRTO layer for target tracking case $(\mathrm{SSE}=49.8799)$. 

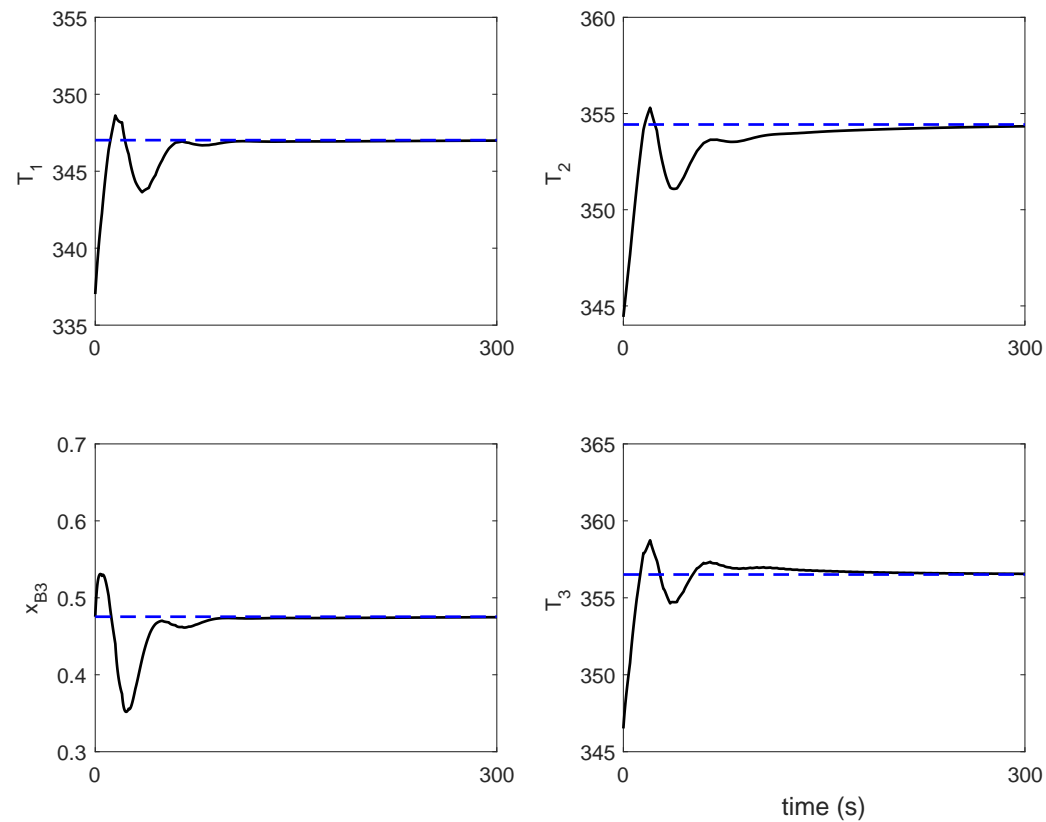

Figure 3.9: Output responses under centralized MPC alone for target tracking case $(\mathrm{SSE}=79.7913)$.
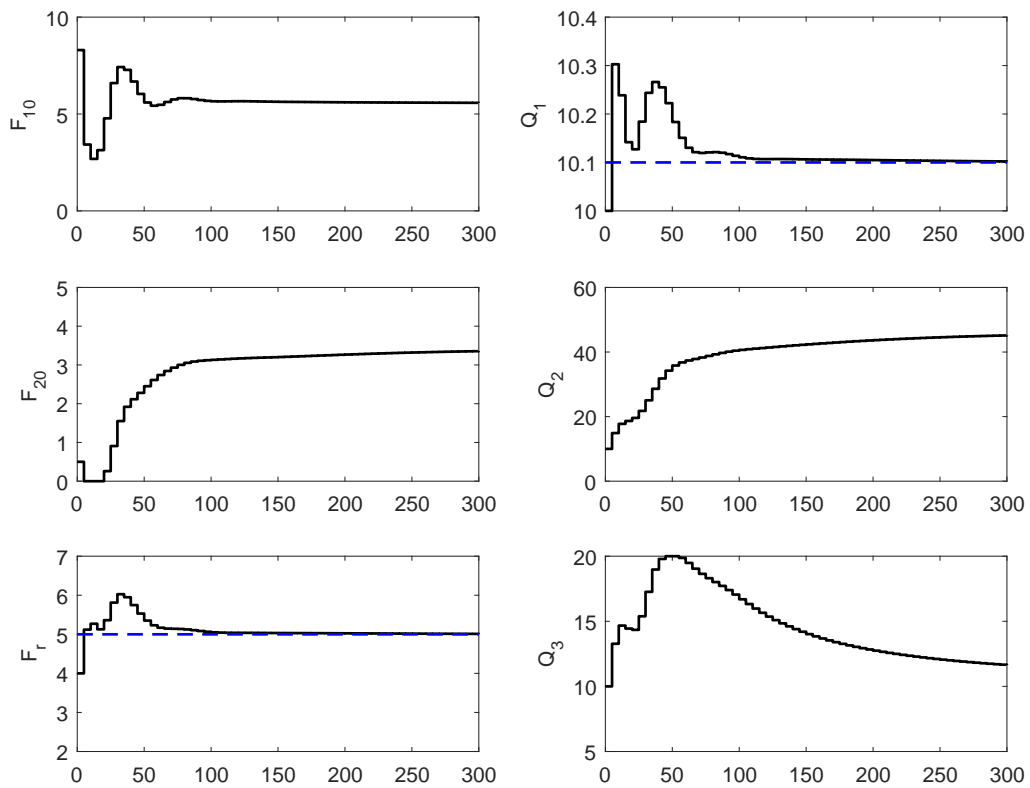

Figure 3.10: Input responses under centralized MPC alone for target tracking case $(\mathrm{SSE}=79.7913)$. 

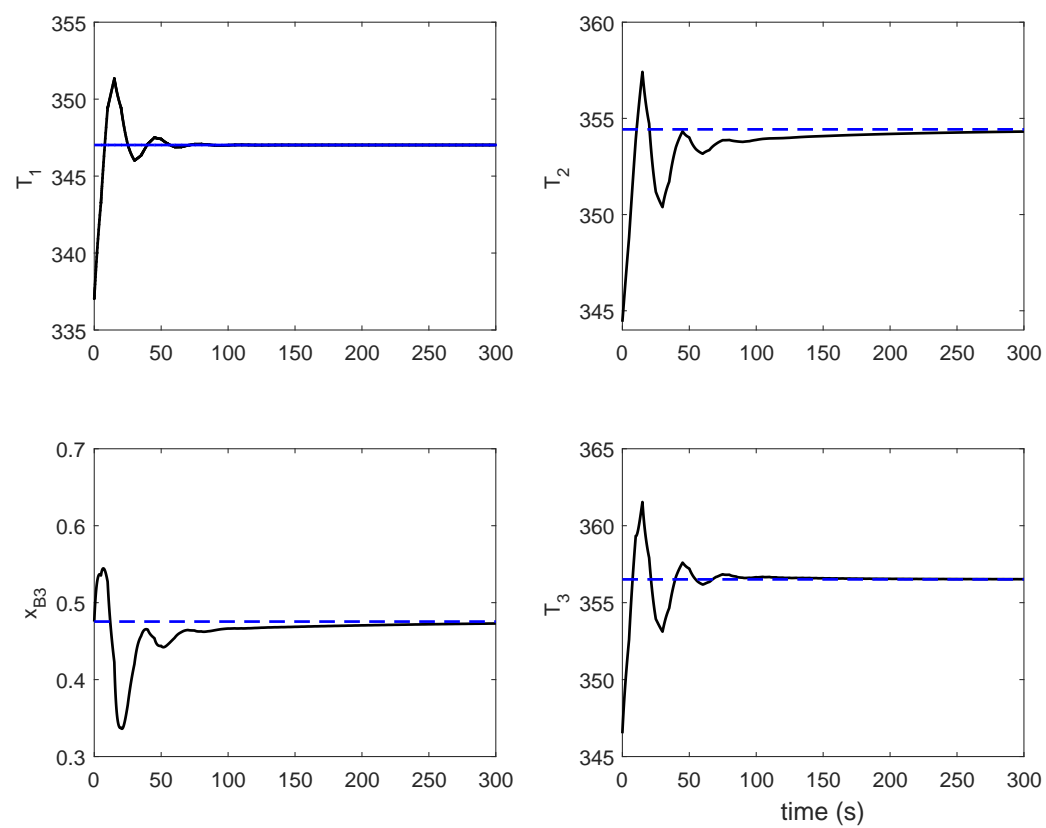

Figure 3.11: Output responses under decentralized MPC for target tracking case $(\mathrm{SSE}=84.3017)$.

counterparts without the DRTO formulation to achieve the desired targets is also addressed in Figs. 3.9 to 3.12 , which show slower responses when the set-points are fixed throughout the horizon. The set-point hold ( $\mathrm{SPH}$ ) method is utilized for the set-point trajectories generated by the DRTO formulation, with set-points held constant over a number of time periods given by $\Delta t_{D R T O} / \Delta t_{M P C}$. The average DRTO execution time for this part of the case study is 0.39 seconds using the CONOPT solver from within AMPL, which is well below the DRTO sample time of $15 \mathrm{~s}$.

Table 3.8 shows the performance measured as the sum of squared errors between the process variables and final targets. While all the scenarios shown here are able to achieve the desired target, the performance varies significantly from case to case. The process responses with the help of the DRTO formulation are substantially better than MPC alone due to the increased flexibility in prescribing set-point trajectories, coupled with the prediction of future interaction effects between the plant and con- 

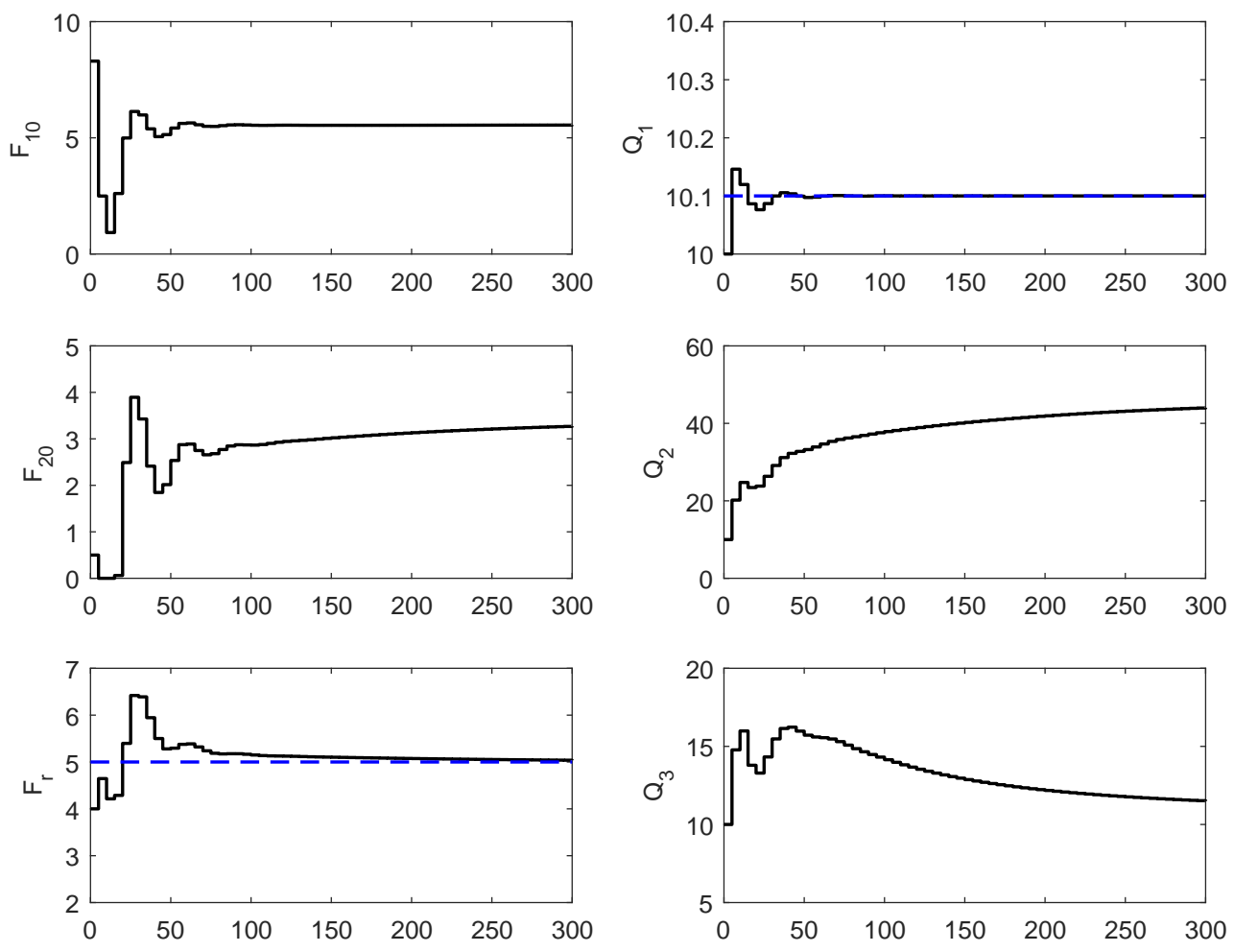

Figure 3.12: Input responses under decentralized MPC for target tracking case (SSE $=84.3017$ ). 
Table 3.7: Design parameters for the DRTO and MPC layers for the target tacking case in Case Study 2.

\begin{tabular}{lllll}
\hline Parameter & Description & $\begin{array}{l}\text { Value } \\
\text { ized })\end{array}$ & $\begin{array}{l}\text { (central- } \\
\text { Value } \\
(\mathrm{MPC} 1)\end{array}$ & $\begin{array}{l}\text { Value } \\
(\mathrm{MPC} 2)\end{array}$ \\
\hline$T_{\text {sim }}^{\text {track }}$ & total simulation time & $300 \mathrm{~s}$ & $300 \mathrm{~s}$ & $300 \mathrm{~s}$ \\
$\Delta t_{D R T O}$ & DRTO sample time & - & $15 \mathrm{~s}$ & $15 \mathrm{~s}$ \\
$\Delta t_{M P C}$ & MPC sample time & $5 \mathrm{~s}$ & $5 \mathrm{~s}$ & $5 \mathrm{~s}$ \\
$p$ & prediction horizon & 5 & 5 & 5 \\
$m$ & control horizon & 2 & 2 & 2 \\
$Q$ & output tracking weight & $\operatorname{diag}\left(1,1,10^{4}, 1\right)$ & 1 & $\operatorname{diag}\left(1,10^{4}, 1\right)$ \\
$R$ & move suppression weight & $\operatorname{diag}(1,1,1,1,1,1)$ & $\operatorname{diag}(1,1)$ & $\operatorname{diag}(1,1,1,1)$ \\
$S$ & control tracking weight & $\operatorname{diag}(0,10,0,0,10,0)$ & $\operatorname{diag}(0,10)$ & $\operatorname{diag}(0,0,10,0)$ \\
$N$ & optimization horizon & - & 12 & 12 \\
$\rho$ & complementarity penalty & - & 1000 & 1000 \\
& parameter & & & \\
\hline
\end{tabular}

Table 3.8: Performance of each scenario for the target tracking case.

\begin{tabular}{lll}
\hline Configuration & Control architecture & SSE Value \\
\hline distributed & MPC + DRTO & 49.8799 \\
centralized & MPC & 79.7913 \\
decentralized & MPC & 84.3017 \\
\hline
\end{tabular}

trollers.

\section{Economic optimization}

The economic optimization of this integrated process can be achieved by formulating an objective function to maximize the profit. The objective function for this part of case study is

$\max \{$ product revenue - cost of raw materials - cost of purge stream - utility costs $\}$ 
which written in terms of process variables becomes

$\min \Delta t_{M P C} \sum_{j=0}^{N-1}-50\left(F_{10 j}+F_{20 j}\right) R_{j}^{1} R_{j}^{2}+\left(F_{10 j}+F_{20 j}\right)+0.01 F_{r j}+5\left(Q_{1 j}+Q_{2 j}+Q_{3 j}\right)$

where $R^{1}$ and $R^{2}$ are defined as hyperbolic tangent switching functions discussed previously. The decision variables and optimization degrees of freedom are as described for the target-tracking case.

The objective function reflects that if the concentration of product $\mathrm{B}, x_{B_{3}}$, stays within specification limits, the revenue will accrue based on the inlet flow rates of raw materials, due to the assumption of perfectly controlled liquid level in all vessels. The utility cost and raw material cost will accumulate throughout the entire simulation time. The design and tuning parameters for this case study are summarized in Table 3.9. The set-point hold ( $\mathrm{SPH}$ ) method is also utilized for the set-point trajectories generated by the DRTO formulation, and held constant over $\Delta t_{D R T O} / \Delta t_{M P C}$ periods.

The plant responses for the coordinated distributed case are shown in Fig. 3.13 for the outputs and Fig. 3.14 for the inputs. The profit accumulated during the simulation time is $\$ 66,113$. The average DRTO execution time is 2.53 seconds. It is apparent that the original steady-state is not at the economic optimum and the DRTO layer is able to generate set-point trajectories to gradually guide the overall process towards a new steady-state condition that is economically optimal. The process tends to minimize the heat provided for each vessel and maximize the feed flow so that the process can operate under lower temperatures while maintaining the concentration needed for saleable product. It is worth noting that the inlet flow rate $F_{20}$ does not reach the maximum value, since there is an economic trade-off between the subsequent evaporation which affects the product quality and product flow rate. We remark that use of a linearized DRTO model may result in the system settling 
Table 3.9: Design parameters for the DRTO and MPC layers for the economic case in Case Study 2.

\begin{tabular}{llll}
\hline Parameter & Description & Value $($ MPC1) & Value (MPC2) \\
\hline$T_{\text {sim }}^{\text {econ }}$ & total simulation time & $240 \mathrm{~s}$ & $240 \mathrm{~s}$ \\
$\Delta t_{D R T O}$ & DRTO sample time & $15 \mathrm{~s}$ & $15 \mathrm{~s}$ \\
$\Delta t_{M P C}$ & MPC sample time & $5 \mathrm{~s}$ & $5 \mathrm{~s}$ \\
$p$ & prediction horizon & 5 & 5 \\
$m$ & control horizon & 2 & 2 \\
$Q$ & output tracking weight & 1 & $\operatorname{diag}\left(1,10^{6}, 1\right)$ \\
$R$ & move suppression weight & $\operatorname{diag}(1,10)$ & $\operatorname{diag}(1,1,1,1)$ \\
$S$ & control tracking weight & $\operatorname{diag}(0,10)$ & $\operatorname{diag}(0,0,10,0)$ \\
$N$ & optimization horizon & 12 & 12 \\
\hline
\end{tabular}

at a suboptimal steady-state operating point. Use of a nonlinear DRTO model for dynamic coordination of distributed MPC systems is explained in subsequent chapter. 

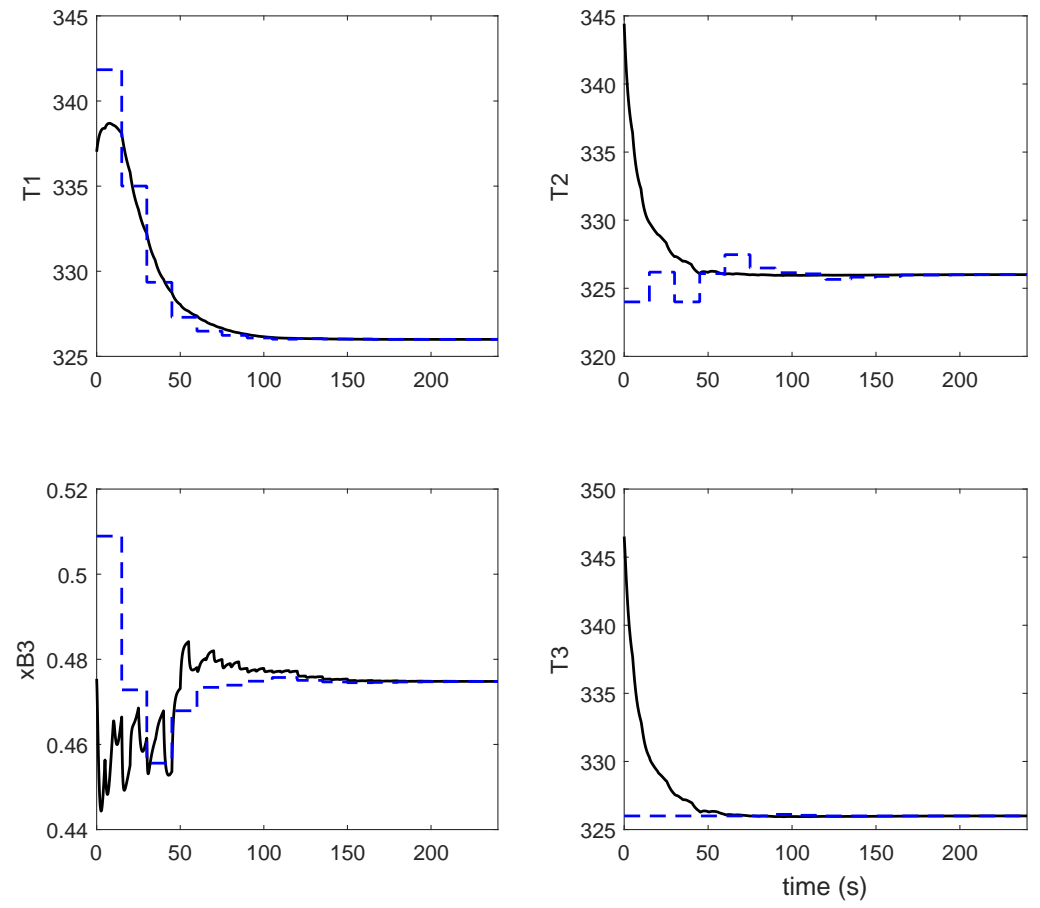

Figure 3.13: Output responses under distributed MPC with DRTO formulation for economic case (profit $=\$ 66,113$ ). 

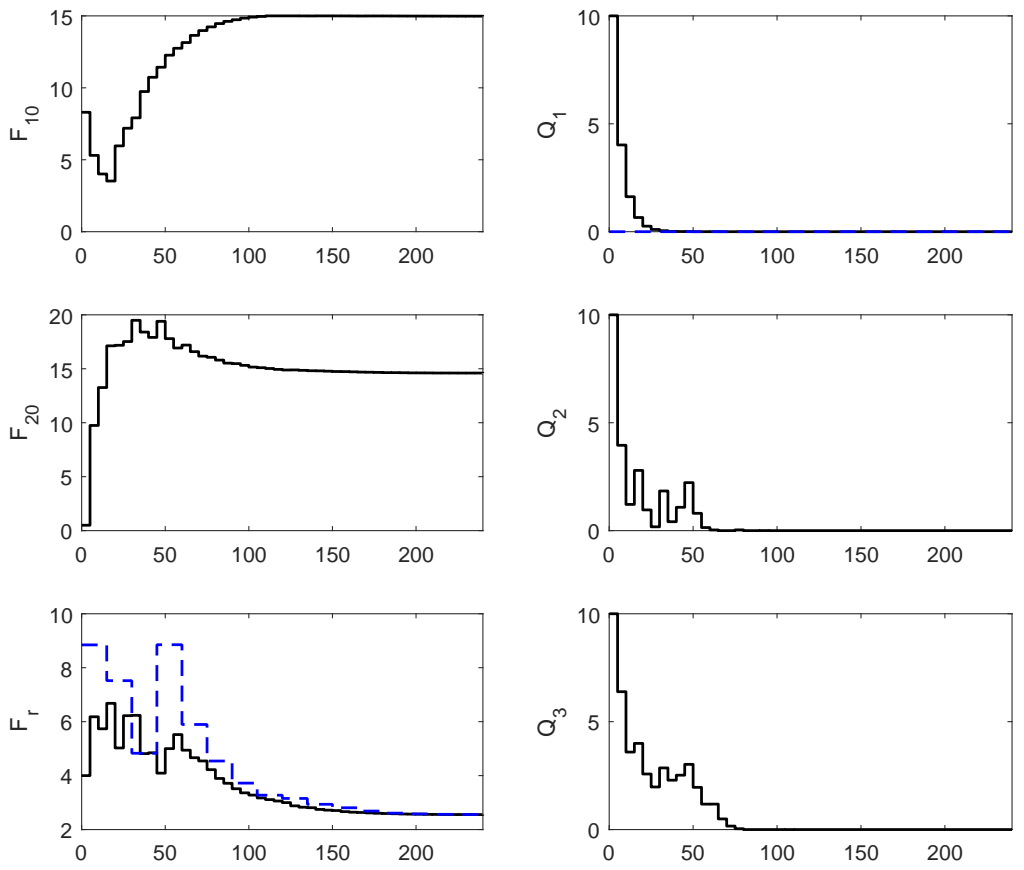

Figure 3.14: Input responses under distributed MPC with DRTO formulation for economic case (profit $=\$ 66,113$ ). 


\section{Chapter 4}

\section{Approximation Techniques}

This chapter introduces two approximation approaches to the rigorous formulation to address the issue of computational complexity. The hybrid formulation uses the rigorous formulation for a short horizon and approximates the feedback for the rest of the horizon. The input clipping method allows the MPC optimization subproblems to be formulated as unconstrained optimization problems and addresses the input constraints when the inputs are applied to the dynamic model using input saturation mechanisms. The detailed formulations are outlined in this chapter and the efficacy of using those approximation techniques demonstrated using both linear and nonlinear case studies.

The formulation and results in this chapter have been submitted to:

Li, H. and Swartz, C. L. (2017). "Approximation techniques of dynamic real-time optimization (DRTO) for distributed MPC systems", Submitted to Computers and Chemical Engineering, in review. 


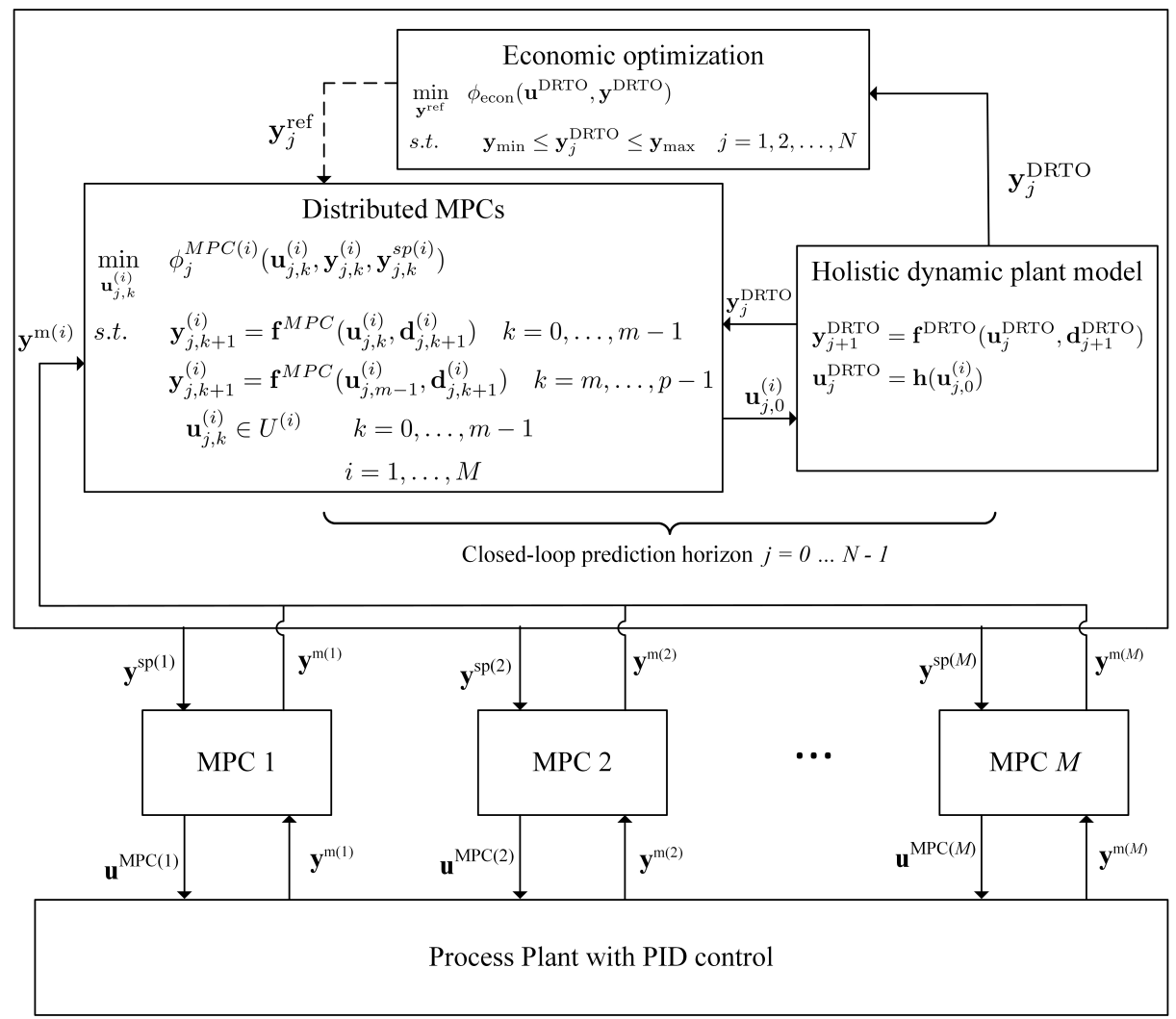

Figure 4.1: The control architecture for coordination of distributed MPCs with CL-DRTO approach

\subsection{Closed-loop DRTO formulation}

The plant models used for the DRTO and MPC formulations are first discussed, after which the rigorous formulation is presented.

The DRTO formulation adopted follows a two layer control structure, as shown in Figure 4.1. The lower MPC layer provides optimal control actions to the underlying process. Such action is calculated based on assigned set-point trajectories from the upper layer. A sequence of MPC optimization subproblems is embedded in the upper layer. A process model of the plant is also incorporated to generate a surrogate plant response for each controller. The overall closed-loop prediction of the process is optimized under a suitably defined objective function. Set-point trajectories are 
calculated as decision variables by the DRTO formulation and subsequently assigned to the lower layer for control tracking.

\subsubsection{Preliminaries: Full-scale and local plant models}

A linear full-scale plant model is available for the DRTO formulation and linear local plant models are available for both the DRTO formulation and MPC formulation. A disturbance estimate corrects the offsets generated by plant-model mismatch. However, a nonlinear model of the plant can be implemented in the primary optimization problem as shown in Jamaludin and Swartz 2016 for application to centralized MPC systems. One topic for future research is to incorporate nonlinear MPCs as the MPC optimization subproblems in the DRTO formulation.

A discrete-time, linear, full-scale plant model is available for the DRTO layer. The plant is assumed to be partitioned into $M$ subsystems, each of which is controlled by a local MPC. The full-scale plant model generates the plant response at each step along the DRTO prediction horizon, $j$ :

$$
\begin{aligned}
& \mathbf{x}_{j+1}^{\operatorname{DRTO}(i)}=A_{i i} \mathbf{x}_{j}^{\operatorname{DRTO}(i)}+B_{i i} \mathbf{u}_{j}^{\operatorname{DRTO}(i)}+\sum_{\substack{h \in M \\
h \neq i}} A_{i h} \mathbf{x}_{j}^{\mathrm{DRTO}(h)}+\sum_{\substack{h \in M \\
h \neq i}} B_{i h} \mathbf{u}_{j}^{\operatorname{DRTO}(h)} \\
& \mathbf{y}_{j}^{\operatorname{DRTO}(i)}=C_{i i} \mathbf{x}_{j}^{\operatorname{DRTO}(i)}+\sum_{\substack{h \in M \\
h \neq i}} C_{i h} \mathbf{x}_{j}^{\operatorname{DRTO}(h)}
\end{aligned}
$$

Each subsystem $i$ has a local plant model for solving the MPC optimization subproblems at the DRTO prediction horizon step $j$. The local plant model can be written 
as:

$$
\begin{aligned}
\mathbf{x}_{j, k+1}^{(i)} & =A_{i i} \mathbf{x}_{j, k}^{(i)}+B_{i i} \mathbf{u}_{j, k}^{(i)} \\
\mathbf{y}_{j, k}^{(i)} & =C_{i i} \mathbf{x}_{j, k}^{(i)}
\end{aligned}
$$

where vectors $\mathbf{x}^{(i)} \in R^{n_{x}^{(i)}}, \mathbf{u}^{(i)} \in R^{n_{u}^{(i)}}$ and $\mathbf{y}^{(i)} \in R^{n_{y}^{(i)}}$ are states, inputs and outputs, respectively, corresponding to the $i$ th subsystem. The diagonal blocks of $A, B$ and $C$ are expressed as $A_{i i}, B_{i i}$ and $C_{i i}$ and are made available for both the full plant model and local plant models. The off-diagonal blocks $A_{i j}, B_{i j}$ and $C_{i j}$ are available exclusively to the primary optimization problem to capture the interactions among subsystems in the generation of the outputs to reflect the behavior of the true plant response used in the DRTO objective function and constraints, and as surrogate plant measurements for the embedded local MPCs used to generate the predicted closed-loop response.

\subsubsection{Rigorous CL-DRTO formulation}

The closed-loop dynamics are simulated in the DRTO formulation through a sequence of MPC optimization subproblems to generate control inputs and applying them at each time instance to produce the plant response. The DRTO optimization formulation, based on the predicted closed-loop response, consequently takes the form of a multilevel optimization problem containing a sequence of MPC QP subproblems. The detailed formulation is presented in Eq.4.5. 


$$
\begin{aligned}
& \min _{\mathbf{y}^{\text {ref }}, \mathbf{u}^{\text {ref }}} \phi^{\mathrm{DRTO}}\left(\mathbf{x}^{\mathrm{DRTO}}, \mathbf{y}^{\mathrm{DRTO}}, \mathbf{u}^{\mathrm{DRTO}}\right) \\
& \text { s.t. } \mathbf{x}_{j+1}^{\operatorname{DRTO}(i)}=A_{i i} \mathbf{x}_{j}^{\operatorname{DRTO}(i)}+B_{i i} \mathbf{u}_{j}^{\operatorname{DRTO}(i)}+\sum_{\substack{h \in M \\
h \neq i}} A_{i h} \mathbf{x}_{j}^{\operatorname{DRTO}(h)}+\sum_{\substack{h \in M \\
h \neq i}} B_{i h} \mathbf{u}_{j}^{\mathrm{DRTO}(h)}, j=0, \ldots, N-1 \\
& \mathbf{y}_{j}^{\operatorname{DRTO}(i)}=C_{i i} \mathbf{x}_{j}^{\operatorname{DRTO}(i)}+\sum_{\substack{h \in M \\
h \neq i}} C_{i h} \mathbf{x}_{j}^{\operatorname{DRTO}(h)}+\mathbf{d}_{j}^{\operatorname{DRTO}(i)}, j=1, \ldots, N \\
& \mathbf{g}^{\mathrm{DRTO}}\left(\mathbf{x}^{\mathrm{DRTO}}, \mathbf{y}^{\mathrm{DRTO}}\right) \geq \mathbf{0} \\
& \mathbf{h}_{i}^{\text {ref }}\left(\mathbf{y}^{\text {ref }}, \mathbf{u}^{\text {ref }}, \mathbf{y}^{\mathrm{sp}(i)}, \mathbf{u}^{\mathrm{sp}(i)}\right)=\mathbf{0}, \quad i=1, \ldots, M \\
& \mathrm{~g}^{\mathrm{ref}}\left(\mathbf{y}^{\mathrm{ref}}, \mathbf{u}^{\mathrm{ref}}\right) \geq \mathbf{0} \\
& \mathbf{u}_{j}^{\mathrm{DRTO}(i)}=\mathbf{u}_{j, 0}^{(i)}, \quad j=0, \ldots, N-1, i=1, \ldots, M \\
& \mathbf{d}_{j}^{\operatorname{DRTO}(i)}=\mathbf{y}^{\mathrm{m}}-C_{i i} \mathbf{x}_{0}^{\operatorname{DRTO}(i)}-\sum_{\substack{h \in M \\
h \neq i}} C_{i h} \mathbf{x}_{0}^{\operatorname{DRTO}(h)}, \quad j=1, \ldots, N \\
& \mathbf{u}_{j, 0}^{(i)} \in \arg \quad \min _{\mathbf{u}_{j, k}^{(i)}} \quad \phi_{j}^{(i)}=\sum_{k=1}^{p}\left(\mathbf{y}_{j, k}^{(i)}-\mathbf{y}_{j, k}^{\mathrm{sp}(i)}\right)^{T} Q^{(i)}\left(\mathbf{y}_{j, k}^{(i)}-\mathbf{y}_{j, k}^{\mathrm{sp}(i)}\right) \\
& +\sum_{k=0}^{m-1}\left(\Delta \mathbf{u}_{j, k}^{(i)}\right)^{T} R^{(i)}\left(\Delta \mathbf{u}_{j, k}^{(i)}\right)+\sum_{k=0}^{m-1}\left(\mathbf{u}_{j, k}^{(i)}-\mathbf{u}_{j, k}^{\mathrm{sp}(i)}\right)^{T} S^{(i)}\left(\mathbf{u}_{j, k}^{(i)}-\mathbf{u}_{j, k}^{\mathrm{sp}(i)}\right) \\
& \text { s.t. } \quad \mathbf{x}_{j, k+1}^{(i)}=A_{i i} \mathbf{x}_{j, k}^{(i)}+B_{i i} \mathbf{u}_{j, k}^{(i)}, \quad k=0, \ldots, m-1 \\
& \mathbf{x}_{j, k+1}^{(i)}=A_{i i} \mathbf{x}_{j, k}^{(i)}+B_{i i} \mathbf{u}_{j, m-1}^{(i)}, \quad k=m, \ldots, p-1 \\
& \mathbf{y}_{j, k}^{(i)}=C_{i i} \mathbf{x}_{j, k}^{(i)}+\mathbf{d}_{j, k}^{(i)}, \quad k=1, \ldots, p \\
& \mathbf{d}_{j, k}^{(i)}=\mathbf{y}^{\mathrm{m}}-C_{i i} \mathbf{x}_{j, 0}^{(i)}, \quad k=1, \ldots, p, \quad j=0 \\
& \mathbf{d}_{j, k}^{(i)}=\mathbf{y}_{j}^{\operatorname{DRTO}(i)}-C_{i i} \mathbf{x}_{j, 0}^{(i)}, \quad k=1, \ldots, p, \quad j>0 \\
& \Delta \mathbf{u}_{j, k}^{(i)}=\mathbf{u}_{j, k}^{(i)}-\mathbf{u}_{j, k-1}^{(i)}, \quad k=0, \ldots, m-1 \\
& \mathbf{u}_{\min }^{(i)} \leq \mathbf{u}_{j, k}^{(i)} \leq \mathbf{u}_{\max }^{(i)}, \quad k=0, \ldots, m-1
\end{aligned}
$$

The output and set-point constraints are addressed in the primary optimization problem and the input constraints are addressed in the MPC optimization subproblems, throughout the entire DRTO prediction horizon. Composite state, input and output vectors for the DRTO plant model are defined as

$$
\begin{aligned}
& \mathbf{x}^{\text {DRTO }}=\left[\left(\mathbf{x}_{0}^{\text {DRTO }}\right)^{T},\left(\mathbf{x}_{1}^{\text {DRTO }}\right)^{T}, \ldots,\left(\mathbf{x}_{N}^{\text {DRTO }}\right)^{T}\right]^{T} \\
& \mathbf{u}^{\text {DRTO }}=\left[\left(\mathbf{u}_{0}^{\text {DRTO }}\right)^{T},\left(\mathbf{u}_{1}^{\text {DRTO }}\right)^{T}, \ldots,\left(\mathbf{u}_{N-1}^{\text {DRTO }}\right)^{T}\right]^{T} \\
& \mathbf{y}^{\text {DRTO }}=\left[\left(\mathbf{y}_{1}^{\text {DRTO }}\right)^{T},\left(\mathbf{y}_{2}^{\text {DRTO }}\right)^{T}, \ldots,\left(\mathbf{y}_{N}^{\text {DRTO }}\right)^{T}\right]^{T}
\end{aligned}
$$


where the $\mathbf{x}_{j}^{\text {DRTO }}, \mathbf{u}_{j}^{\text {DRTO }}$ and $\mathbf{y}_{j}^{\text {DRTO }}$ comprise their collective subsystem states, inputs and outputs, which for the states can be written as

$$
\mathbf{x}_{j}^{\mathrm{DRTO}}=\left[\left(\mathbf{x}_{j}^{\mathrm{DRTO}(1)}\right)^{T}, \ldots,\left(\mathbf{x}_{j}^{\mathrm{DRTO}(M)}\right)^{T}\right]^{T}
$$

with analogous expressions for the inputs and outputs.

The composite output and input reference trajectories over the DRTO prediction horizon $N, \mathbf{y}^{\text {ref }}$ and $\mathbf{u}^{\text {ref }}$, respectively, are the main decision variables and comprise the degrees of freedom in the primary optimization problem. The set-point trajectories for the MPC subproblems $\mathbf{y}_{j, k}^{\mathrm{sp}(i)}$ and $\mathbf{u}_{j, k}^{\mathrm{sp}(i)}$ are extracted from these reference trajectories along the DRTO prediction horizon. The constraints $\mathbf{h}_{i}^{\text {ref }}$ describe the mapping between the reference and set-point trajectories, shown in more detail as follows. Consider $\mathbf{y}_{j}^{\text {ref }}$ and $\mathbf{u}_{j}^{\text {ref }}$ as the reference trajectories corresponding to DRTO time step $j$. The extraction of the set-point trajectory for MPC subproblem $j$ and subsystem $i$ from the reference trajectory can then be represented as

$$
\begin{aligned}
& \mathbf{y}_{j, k}^{\mathrm{sp}(i)}=E_{y}^{(i)} \mathbf{y}_{j+k}^{\mathrm{ref}}, \quad k=1, \ldots, p \\
& \mathbf{u}_{j, k}^{\mathrm{sp}(i)}=E_{u}^{(i)} \mathbf{u}_{j+k}^{\mathrm{ref}}, \quad k=0, \ldots, m-1
\end{aligned}
$$

where the matrices $E_{y}^{(i)} \in R^{n_{y}^{(i)} \times n_{y}}$ and $E_{u}^{(i)} \in R^{n_{u}^{(i)} \times n_{u}}$ map the full plant outputs and inputs to the subsystem outputs and inputs. The extraction of the MPC setpoint trajectory from the composite reference trajectory is illustrated in Fig 4.2 for a centralized MPC configuration.

The reference trajectory for a particular output or input has a unique value for each sample interval along the DRTO horizon. However, portions of the reference trajectory can correspond to different set-point trajectories, depending on the position along the DRTO horizon of the MPC subproblems to which the set-point trajectories apply, as well as the length of the MPC prediction and/or control move horizon. It 


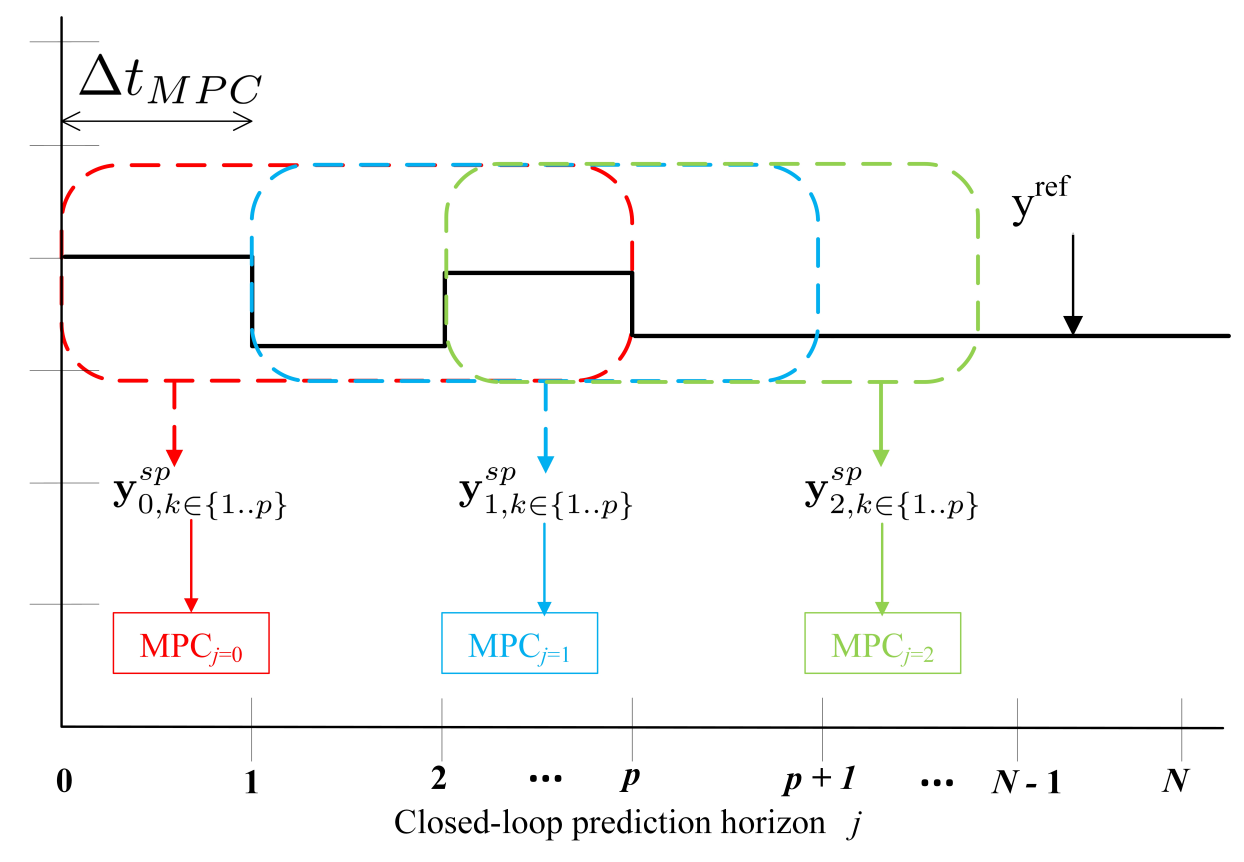

Figure 4.2: The illustration of set-point extraction from reference trajectory.

is noted that the extension of reference values beyond $N$ are required for the MPC subproblems toward the end of the DRTO horizon, and are set equal to the values at $N$. The output and state constraints are contained in $\mathbf{g}^{\text {DRTO }}$, and constraints on the reference trajectories are applied as $\mathbf{g}^{\text {ref }}$.

Each distributed MPC generates sets of control actions, $\mathbf{u}_{j, k}^{(i)}$, at each DRTO step $j$, the first of which $\mathbf{u}_{j, 0}^{(i)}$ are equated to the inputs of primary optimization problem, $\mathbf{u}_{j}^{\mathrm{DRTO}(i)}$, at that time instance:

$$
\mathbf{u}_{j}^{\operatorname{DRTO}(i)}=\mathbf{u}_{j, 0}^{(i)}, \quad j=0, \ldots, N-1
$$

Disturbance estimates are computed for both the DRTO plant model and the MPC subproblems. The first disturbance estimate is computed as the difference between the outputs measured immediately prior to the DRTO execution and predicted output values based on information at the previous time step. The disturbance estimate for 
subsequent MPC problems along the DRTO horizon utilize the outputs generated by the full DRTO plant model as surrogate measurements. The initial states in 4.5 may be obtained by advancing the states corresponding to the previous time step through the relevant dynamic model.

Alternative disturbance handling, state estimation and MPC formulation schemes can be readily implemented in the proposed formulation. In order to eliminate degrees of freedom for a non-square system, the input tracking term is included in the MPC objective function, with the input set-points corresponding to ideal resting values Qin and Badgwell, 2003]. The output constraints for MPCs are implemented at the primary DRTO level rather than in the MPC subproblems, as the latter may contribute to problems of infeasibility and/or instability Zafiriou and Marchal, 1991.

\subsection{Closed-Loop Approximation}

This section provides details of two strategies for approximating the closed-loop response of the plant under constrained, distributed MPC, with the goal of reducing computation time while maintaining a high degree of accuracy.

\subsubsection{Hybrid CL-DRTO formulation}

The hybrid formulation incorporates rigorous closed-loop prediction for a limited DRTO prediction horizon and then uses a single MPC optimization subproblem for the remainder of the horizon. We denote the number of MPC sample intervals for which feedback is accounted for in the DRTO horizon as $n$. The relation between the control inputs applied to the full DRTO plant model and those generated by the 
MPC subproblems now takes the form,

$$
\begin{aligned}
& \mathbf{u}_{j}^{\mathrm{DRTO}(i)}=\mathbf{u}_{j, 0}^{(i)}, \quad i=1, \ldots, M, \quad j=0, \ldots, n-1 \\
& \mathbf{u}_{n+k}^{\mathrm{DRTO}(i)}=\overline{\mathbf{u}}_{k}^{(i)}, \quad k=0, \ldots, N-n-1
\end{aligned}
$$

where $\overline{\mathbf{u}}_{k}^{(i)}$ denotes the control inputs calculated by the last MPC optimization subproblem, which is formulated in the same way as the previous MPC subproblems in the sequence, but with the prediction and control move horizons extended to $N-n$ in order to provide sufficient control inputs for the DRTO plant model for the rest of the DRTO prediction horizon. We use an overbar to denote the variables associated with the last MPC subproblem, and note that only one subscript is necessary for the states, outputs and inputs since no further MPC subproblems are defined over the DRTO horizon.

The detailed formulation for the hybrid formulation is organized as Eq. 4.12). 


$$
\begin{aligned}
& \min _{\mathbf{y}^{\text {ref }}, \mathbf{u}^{\text {ref }}} \phi^{\text {DRTO }}\left(\mathbf{x}^{\text {DRTO }}, \mathbf{y}^{\text {DRTO }}, \mathbf{u}^{\text {DRTO }}\right) \\
& \text { s.t. } \mathbf{x}_{j+1}^{\mathrm{DRTO}(i)}=A_{i i} \mathbf{x}_{j}^{\mathrm{DRTO}(i)}+B_{i i} \mathbf{u}_{j}^{\mathrm{DRTO}(i)}+\sum_{\substack{h \in M \\
h \neq i}} A_{i h} \mathbf{x}_{j}^{\mathrm{DRTO}(h)}+\sum_{\substack{h \in M \\
h \neq i}} B_{i h} \mathbf{u}_{j}^{\mathrm{DRTO}(h)}, j=0, \ldots, N-1 \\
& \mathbf{y}_{j}^{\mathrm{DRTO}(i)}=C_{i i} \mathbf{x}_{j}^{\mathrm{DRTO}(i)}+\sum_{\substack{h \in M \\
h \neq i}} C_{i h} \mathbf{x}_{j}^{\mathrm{DRTO}(h)}+\mathbf{d}_{j}^{\mathrm{DRTO}(i)}, j=1, \ldots, N \\
& \mathbf{g}^{\mathrm{DRTO}}\left(\mathbf{x}_{j}^{\mathrm{DRTO}}, \mathbf{y}_{j}^{\mathrm{DRTO}}\right) \geq \mathbf{0} \\
& \mathbf{h}^{\text {ref }}\left(\mathbf{y}^{\text {ref }}, \mathbf{u}^{\text {ref }}, \mathbf{y}^{\mathrm{sp}(i)}, \mathbf{u}^{\mathrm{sp}(i)}\right)=\mathbf{0}, \quad i=1, \ldots, M \\
& \mathbf{g}^{\text {ref }}\left(\mathbf{y}_{j}^{\text {ref }}, \mathbf{u}_{j}^{\text {ref }}\right) \geq \mathbf{0}, \quad j=0, \ldots, N-1 \\
& \mathbf{u}_{j}^{\operatorname{DRTO}(i)}=\mathbf{u}_{j, 0}^{(i)}, \quad j=0, \ldots, n-1 \\
& \mathbf{u}_{j+k}^{\mathrm{DRTO}(i)}=\overline{\mathbf{u}}_{k}^{(i)}, \quad j=n, \quad k=0, \ldots, N-n-1 \\
& \mathbf{d}_{j}^{\mathrm{DRTO}(i)}=\mathbf{y}^{\mathrm{m}}-C_{i i} \mathbf{x}_{0}^{\operatorname{DRTO}(i)}-\sum_{\substack{h \in M \\
h \neq i}} C_{i h} \mathbf{x}_{0}^{\operatorname{DRTO}(h)}, j=1, \ldots, N \\
& \mathbf{u}_{j, 0}^{(i)} \in \arg \min _{\mathbf{u}_{j, k}^{(i)}} \phi_{j}^{(i)}=\sum_{k=1}^{p}\left(\mathbf{y}_{j, k}^{(i)}-\mathbf{y}_{j, k}^{\mathrm{sp}(i)}\right)^{T} Q^{(i)}\left(\mathbf{y}_{j, k}^{(i)}-\mathbf{y}_{j, k}^{\mathrm{sp}(i)}\right) \\
& +\sum_{k=0}^{m-1}\left(\Delta \mathbf{u}_{j, k}^{(i)}\right)^{T} R^{(i)}\left(\Delta \mathbf{u}_{j, k}^{(i)}\right)+\sum_{k=0}^{m-1}\left(\mathbf{u}_{j, k}^{(i)}-\mathbf{u}_{j, k}^{\mathrm{sp}(i)}\right)^{T} S^{(i)}\left(\mathbf{u}_{j, k}^{(i)}-\mathbf{u}_{j, k}^{\mathrm{sp}(i)}\right) \\
& \text { s.t. } \quad \mathbf{x}_{j, k+1}^{(i)}=A^{(i)} \mathbf{x}_{j, k}^{(i)}+B^{(i)} \mathbf{u}_{j, k}^{(i)}, \quad k=0, \ldots, m-1 \\
& \mathbf{x}_{j, k+1}^{(i)}=A_{i i} \mathbf{x}_{j, k}^{(i)}+B_{i i} \mathbf{u}_{j, m-1}^{(i)}, \quad k=m, \ldots, p-1 \\
& \mathbf{y}_{j, k}^{(i)}=C_{i i} \mathbf{x}_{j, k}^{(i)}+\mathbf{d}_{j, k}^{(i)}, \quad k=1, \ldots, p \\
& \mathbf{d}_{j, k}^{(i)}=\mathbf{y}^{\mathrm{m}}-C_{i i} \mathbf{x}_{j, 0}^{(i)}, \quad k=1, \ldots, p, \quad j=0 \\
& \mathbf{d}_{j, k}^{(i)}=\mathbf{y}_{j}^{\operatorname{DRTO}(i)}-C_{i i} \mathbf{x}_{j, 0}^{(i)}, \quad k=1, \ldots, p \\
& \Delta \mathbf{u}_{j, k}^{(i)}=\mathbf{u}_{j, k}^{(i)}-\mathbf{u}_{j, k-1}^{(i)}, \quad k=0, \ldots, m-1 \\
& \mathbf{u}_{\min }^{(i)} \leq \mathbf{u}_{j, k}^{(i)} \leq \mathbf{u}_{\max }^{(i)}, \quad k=0, \ldots, m-1 \\
& \overline{\mathbf{u}}^{(i)} \in \arg \quad \min _{\overline{\mathbf{u}}^{(i)}} \quad \phi^{(i)}=\sum_{k=1}^{N-n}\left(\overline{\mathbf{y}}_{k}^{(i)}-\overline{\mathbf{y}}_{k}^{\mathrm{sp}(i)}\right)^{T} Q^{(i)}\left(\overline{\mathbf{y}}_{k}^{(i)}-\overline{\mathbf{y}}_{k}^{\mathrm{sp}(i)}\right) \\
& +\sum_{k=0}^{N-n-1}\left(\Delta \overline{\mathbf{u}}_{k}^{(i)}\right)^{T} R^{(i)}\left(\Delta \overline{\mathbf{u}}_{k}^{(i)}\right)+\sum_{k=0}^{N-n-1}\left(\overline{\mathbf{u}}_{k}^{(i)}-\overline{\mathbf{u}}_{k}^{\mathrm{sp}(i)}\right)^{T} S^{(i)}\left(\overline{\mathbf{u}}_{k}^{(i)}-\overline{\mathbf{u}}_{k}^{\mathrm{sp}(i)}\right) \\
& \text { s.t. } \quad \overline{\mathbf{x}}_{k+1}^{(i)}=A_{i i} \overline{\mathbf{x}}_{k}^{(i)}+B_{i i} \overline{\mathbf{u}}_{k}^{(i)}, \quad k=0, \ldots, N-n-1 \\
& \overline{\mathbf{y}}_{k}^{(i)}=C_{i i} \overline{\mathbf{x}}_{k}^{(i)}+\overline{\mathbf{d}}_{k}^{(i)}, \quad k=1, \ldots, N-n \\
& \overline{\mathbf{d}}_{k}^{(i)}=\mathbf{y}_{j}^{\operatorname{DRTO}(i)}-C_{i i} \mathbf{x}_{j, 0}^{(i)}, \quad k=1, \ldots, N-n \\
& \Delta \overline{\mathbf{u}}_{k}^{(i)}=\overline{\mathbf{u}}_{k}^{(i)}-\overline{\mathbf{u}}_{k-1}^{(i)}, \quad k=0, \ldots, N-n-1 \\
& \mathbf{u}_{\min }^{(i)} \leq \overline{\mathbf{u}}_{k}^{(i)} \leq \mathbf{u}_{\max }^{(i)}, \quad k=0, \ldots, N-n-1 \\
& i=1, \ldots, M \\
& j=n
\end{aligned}
$$




\subsubsection{Input clipping formulation}

Instead of constrained MPC optimization subproblems embedded along the DRTO prediction horizon, the input clipping formulation uses unconstrained MPC coupled with an input saturation mechanism. The controller output $\mathbf{u}_{j}^{\mathrm{MPC}(i)}$ for subsystem $i$ computed by the unconstrained MPC optimization subproblems and the DRTO input $\mathbf{u}_{j}^{\mathrm{DRTO}(i)}$ to be implemented to the dynamic process follow the relationship expressed as

$$
\mathbf{u}_{j}^{\operatorname{DRTO}(i)}=\left\{\begin{array}{lc}
\mathbf{u}_{\min }^{(i)}, & \mathbf{u}_{j}^{\operatorname{MPC}(i)}<\mathbf{u}_{\min }^{(i)} \\
\mathbf{u}_{j}^{\operatorname{MPC}(i)}, & \mathbf{u}_{\min }^{(i)} \leq \mathbf{u}_{j}^{\operatorname{MPC}(i)} \leq \mathbf{u}_{\max }^{(i)} \\
\mathbf{u}_{\max }^{(i)}, & \mathbf{u}_{j}^{\operatorname{MPC}(i)}>\mathbf{u}_{\max }^{(i)}
\end{array}\right.
$$

The conditional expression above can be combined into a continuous formulation with complementarity constraints, as proposed by Baker and Swartz 2004, which we show in the next section to be readily solvable using a standard NLP solver. Slack variables, $\boldsymbol{\eta}_{j}^{1(i)}$ and $\boldsymbol{\eta}_{j}^{2(i)}$ are introduced for each DRTO prediction step $j$ and MPC subsystem $i$ :

$$
\mathbf{u}_{j}^{\mathrm{DRTO}(i)}=\mathbf{u}_{j}^{\mathrm{MPC}(i)}+\boldsymbol{\eta}_{j}^{1(i)}-\boldsymbol{\eta}_{j}^{2(i)}
$$

The constraint set is then formulated such that the slack variables corresponding to an input variable are both zero if the MPC input is within its constraint bounds, and of not, the slack variable corresponding to the bound that is violated would be positive ( $\boldsymbol{\eta}_{j}^{1(i)}$ for the minimum bound and $\boldsymbol{\eta}_{j}^{1(i)}$ for the maximum), forcing the DRTO input to take on the value of the constraint limit through a complementarity constraint. 
This is achieved with the formulation,

$$
\begin{aligned}
\mathbf{u}_{j}^{\mathrm{MPC}(i)}+\boldsymbol{\eta}_{j}^{1(i)}-\boldsymbol{\eta}_{j}^{2(i)} & =\mathbf{u}_{j}^{\mathrm{DRTO}(i)} \\
\mathbf{u}_{j}^{\mathrm{MPC}(i)}+\boldsymbol{\eta}_{j}^{1(i)}-\boldsymbol{\eta}_{j}^{2(i)} & =\mathbf{u}_{j}^{\mathrm{DRTO}(i)} \\
\mathbf{u}_{j}^{\mathrm{DRTO}(i)}-\mathbf{u}_{\mathrm{min}}^{(i)} & =\boldsymbol{\mu}_{j}^{1(i)} \\
\mathbf{u}_{\max }^{(i)}-\mathbf{u}_{j}^{\mathrm{DRTO}(i)} & =\boldsymbol{\mu}_{j}^{2(i)} \\
\left(\boldsymbol{\eta}_{j}^{1(i)}\right)^{T} \boldsymbol{\mu}_{j}^{1(i)} & =0 \\
\left(\boldsymbol{\eta}_{j}^{2(i)}\right)^{T} \boldsymbol{\mu}_{j}^{2(i)} & =0 \\
\left(\boldsymbol{\eta}_{j}^{1(i)}, \boldsymbol{\eta}_{j}^{2(i)}, \boldsymbol{\mu}_{j}^{1(i)}, \boldsymbol{\mu}_{j}^{2(i)}\right) & \geq \mathbf{0}
\end{aligned}
$$

Eq. 4.16) shows the input clipping formulation applied to the rigorous formulation presented previously. The input saturation mechanism can also be implemented in the hybrid formulation. 


$$
\begin{aligned}
& \min _{\mathbf{y}^{\text {ref }}, \mathbf{u}^{\text {ref }}} \phi^{\mathrm{DRTO}}\left(\mathbf{x}^{\mathrm{DRTO}}, \mathbf{y}^{\mathrm{DRTO}}, \mathbf{u}^{\mathrm{DRTO}}\right) \\
& \text { s.t. } \mathbf{x}_{j+1}^{\operatorname{DRTO}(i)}=A_{i i} \mathbf{x}_{j}^{\operatorname{DRTO}(i)}+B_{i i} \mathbf{u}_{j}^{\operatorname{DRTO}(i)}+\sum_{\substack{h \in M \\
h \neq i}} A_{i h} \mathbf{x}_{j}^{\operatorname{DRTO}(h)}+\sum_{\substack{h \in M \\
h \neq i}} B_{i h} \mathbf{u}_{j}^{\mathrm{DRTO}(h)}, j=0, \ldots, N-1 \\
& \mathbf{y}_{j}^{\operatorname{DRTO}(i)}=C_{i i} \mathbf{x}_{j}^{\operatorname{DRTO}(i)}+\sum_{\substack{h \in M \\
h \neq i}} C_{i h} \mathbf{x}_{j}^{\operatorname{DRTO}(h)}+\mathbf{d}_{j}^{\operatorname{DRTO}(i)}, j=1, \ldots, N \\
& \mathbf{g}^{\mathrm{DRTO}}\left(\mathbf{x}^{\mathrm{DRTO}}, \mathbf{y}^{\mathrm{DRTO}}\right) \geq \mathbf{0} \\
& \mathbf{h}_{i}^{\mathrm{ref}}\left(\mathbf{y}^{\mathrm{ref}}, \mathbf{u}^{\mathrm{ref}}, \mathbf{y}^{\mathrm{sp}(i)}, \mathbf{u}^{\mathrm{sp}(i)}\right)=\mathbf{0}, \quad i=1, \ldots, M \\
& \mathrm{~g}^{\mathrm{ref}}\left(\mathbf{y}^{\mathrm{ref}}, \mathbf{u}^{\mathrm{ref}}\right) \geq \mathbf{0} \\
& \mathbf{u}_{j}^{\mathrm{DRTO}(i)}=\mathbf{u}_{j, 0}^{(i)}, \quad j=0, \ldots, N-1, i=1, \ldots, M \\
& \mathbf{d}_{j}^{\mathrm{DRTO}(i)}=\mathbf{y}^{\mathrm{m}}-C_{i i} \mathbf{x}_{0}^{\mathrm{DRTO}(i)}-\sum_{\substack{h \in M \\
h \neq i}} C_{i h} \mathbf{x}_{0}^{\mathrm{DRTO}(h)}, \quad j=1, \ldots, N \\
& \mathbf{u}_{j}^{\mathrm{MPC}(i)}=\mathbf{u}_{j, 0}^{(i)}, \quad j=0, \ldots, N-1 \\
& \mathbf{u}_{j}^{\mathrm{MPC}(i)}+\boldsymbol{\eta}_{j}^{1(i)}-\boldsymbol{\eta}_{j}^{2(i)}=\mathbf{u}_{j}^{\mathrm{DRTO}(i)} \\
& \mathbf{u}_{j}^{\mathrm{DRTO}(i)}-\mathbf{u}_{\mathrm{min}}^{(i)}=\boldsymbol{\mu}_{j}^{1(i)} \\
& \mathbf{u}_{\max }^{(i)}-\mathbf{u}_{j}^{\mathrm{DRTO}(i)}=\boldsymbol{\mu}_{j}^{2(i)} \\
& \left(\boldsymbol{\eta}_{j}^{1(i)}\right)^{T} \boldsymbol{\mu}_{j}^{1(i)}=0 \\
& \left(\boldsymbol{\eta}_{j}^{2(i)}\right)^{T} \boldsymbol{\mu}_{j}^{2(i)}=0 \\
& \left(\boldsymbol{\eta}_{j}^{1(i)}, \boldsymbol{\eta}_{j}^{2(i)}, \boldsymbol{\mu}_{j}^{1(i)}, \boldsymbol{\mu}_{j}^{2(i)}\right) \geq \mathbf{0} \\
& \mathbf{u}_{j, 0}^{(i)} \in \arg \min _{\mathbf{u}_{j, k}^{(i)}} \phi_{j}^{(i)}=\sum_{k=1}^{p}\left(\mathbf{y}_{j, k}^{(i)}-\mathbf{y}_{j, k}^{\mathrm{sp}(i)}\right)^{T} Q^{(i)}\left(\mathbf{y}_{j, k}^{(i)}-\mathbf{y}_{j, k}^{\mathrm{sp}(i)}\right) \\
& +\sum_{k=0}^{m-1}\left(\Delta \mathbf{u}_{j, k}^{(i)}\right)^{T} R^{(i)}\left(\Delta \mathbf{u}_{j, k}^{(i)}\right)+\sum_{k=0}^{m-1}\left(\mathbf{u}_{j, k}^{(i)}-\mathbf{u}_{j, k}^{\mathrm{sp}(i)}\right)^{T} S^{(i)}\left(\mathbf{u}_{j, k}^{(i)}-\mathbf{u}_{j, k}^{\mathrm{sp}(i)}\right) \\
& \text { s.t. } \quad \mathbf{x}_{j, k+1}^{(i)}=A_{i i} \mathbf{x}_{j, k}^{(i)}+B_{i i} \mathbf{u}_{j, k}^{(i)}, \quad k=0, \ldots, m-1 \\
& \mathbf{x}_{j, k+1}^{(i)}=A_{i i} \mathbf{x}_{j, k}^{(i)}+B_{i i} \mathbf{u}_{j, m-1}^{(i)}, \quad k=m, \ldots, p-1 \\
& \mathbf{y}_{j, k}^{(i)}=C_{i i} \mathbf{x}_{j, k}^{(i)}+\mathbf{d}_{j, k}^{(i)}, \quad k=1, \ldots, p \\
& \mathbf{d}_{j, k}^{(i)}=\mathbf{y}^{\mathrm{m}}-C_{i i} \mathbf{x}_{j, 0}^{(i)}, \quad k=1, \ldots, p, \quad j=0 \\
& \mathbf{d}_{j, k}^{(i)}=\mathbf{y}_{j}^{\operatorname{DRTO}(i)}-C_{i i} \mathbf{x}_{j, 0}^{(i)}, \quad k=1, \ldots, p, \quad j>0 \\
& \Delta \mathbf{u}_{j, k}^{(i)}=\mathbf{u}_{j, k}^{(i)}-\mathbf{u}_{j, k-1}^{(i)}, \quad k=0, \ldots, m-1 \\
& \begin{array}{c}
i=1, \ldots, M \\
j=0, \ldots, N-1
\end{array}
\end{aligned}
$$




\subsection{Solution approach}

In this section, we describe a solution strategy for the multilevel DRTO problem, as well as two approaches for determining the solution to the unconstrained MPC optimization problem utilized in the input clipping approximation method.

\subsubsection{Karush-Kuhn-Tucker(KKT) optimality conditions}

The proposed formulation can be solved in principle by adopting a sequential solution approach involving a succession of closed-loop simulations. However, in order to avoid potential difficulties with nonsmoothness induced by the MPC input constraints, a simultaneous solution approach is followed in this work. The MPC subproblems are replaced by their equivalent first-order Karush-Kuhn-Tucker(KKT) optimality conditions, which results in a single-level mathematical program with complementarity constraints (MPCC) [Baker and Swartz, 2008]. This transformation can be illustrated using the following general QP formulation,

$$
\begin{array}{ll}
\min _{z} & \frac{1}{2} \mathbf{z}^{T} H \mathbf{z}+\mathbf{g}^{T} \mathbf{z} \\
\text { s.t. } & A \mathbf{z}=\mathbf{b}, \\
& \mathbf{z} \geq \mathbf{0}
\end{array}
$$

whose KKT optimality conditions are given by,

$$
\begin{aligned}
& H \mathbf{z}+\mathbf{g}-A^{T} \boldsymbol{\nu}-\boldsymbol{\eta}=\mathbf{0} \\
& A \mathbf{z}-\mathbf{b}=\mathbf{0}, \\
& z_{i} \eta_{i}=0, \quad i \in C \\
& (\mathbf{z}, \boldsymbol{\eta}) \geq \mathbf{0}
\end{aligned}
$$


The set of constraints derived from MPC optimization subproblems contains complementarity constraints that may pose difficulties to nonlinear programming (NLP) algorithms; however, effective strategies for dealing with them have been developed, based on modifications to the NLP algorithm or problem formulation Baumrucker et al. 2008. Here, we follow an exact penalty approach Ralph and Wright, 2004], where the complementarity constraints are removed from the constraint set and included instead in the objective function as a penalty term,

$$
\phi^{\mathrm{DRTO}}+\rho \sum_{k=1}^{N} \mathbf{z}_{k}^{T} \boldsymbol{\eta}_{k}
$$

where $\rho$ is a penalty parameter.

\subsubsection{Analytical solution of unconstrained MPC problem}

While the unconstrained MPC optimization subproblems arising in the input clipping formulation can be formulated using their KKT conditions as before (without the input constraint Lagrange multipliers), the analytical solution of the unconstrained MPC problem can be directly embedded in the DRTO formulation. By using the analytical solution, the problem dimension for the DRTO formulation can be further reduced due to the elimination of the state variables and Lagrange multipliers corresponding to the MPC optimization subproblems. 
Consider the unconstrained formulation of the MPC problem, represented as

$$
\begin{gathered}
\min _{\mathbf{u}_{k}} \phi=\sum_{k=1}^{p}\left(\mathbf{y}_{k}-\mathbf{y}_{k}^{\mathrm{sp}}\right)^{T} Q\left(\mathbf{y}_{k}-\mathbf{y}_{k}^{\mathrm{sp}}\right)+\sum_{k=0}^{m-1}\left(\Delta \mathbf{u}_{k}\right)^{T} R\left(\Delta \mathbf{u}_{k}\right)+ \\
\sum_{k=0}^{m-1}\left(\mathbf{u}_{k}-\mathbf{u}_{k}^{\mathrm{sp}}\right)^{T} S\left(\mathbf{u}_{k}-\mathbf{u}_{k}^{\mathrm{sp}}\right) \\
\text { s.t. } \quad \mathbf{x}_{k+1}=A \mathbf{x}_{k}+B \mathbf{u}_{k}, \quad k=0, \ldots, m-1 \\
\mathbf{x}_{k+1}=A \mathbf{x}_{k}+B \mathbf{u}_{m-1}, \quad k=m, \ldots, p-1 \\
\mathbf{y}_{k}=C \mathbf{x}_{k}+\mathbf{d}_{k}, \quad k=1, \ldots, p \\
\Delta \mathbf{u}_{k}=\mathbf{u}_{k}-\mathbf{u}_{k-1}, \quad k=0, \ldots, m-1
\end{gathered}
$$

The variables and parameters used above are consistent with those used in the previous section, but for clarity the plant subsystem and DRTO time step indices have been omitted. Direct solution of (4.20) through the KKT system involves the solution of a linear equation system in the state, input and output variables, and Lagrange multipliers corresponding to the equality constraints. An alternative approach is to reformulate 4.20 in terms of input changes as the optimization decision variables, with elimination of the states through the equality constraints. The resulting problem can be stated as

$$
\begin{aligned}
& \min _{\Delta \tilde{\mathbf{u}}} \tilde{\phi}=\left(\tilde{\mathbf{y}}-\tilde{\mathbf{y}}^{\mathrm{sp}}\right)^{T} \tilde{Q}\left(\tilde{\mathbf{y}}-\tilde{\mathbf{y}}^{\mathrm{sp}}\right)+(\Delta \tilde{\mathbf{u}})^{T} \tilde{R}(\Delta \tilde{\mathbf{u}})+ \\
&\left(\tilde{\mathbf{u}}-\tilde{\mathbf{u}}^{\mathrm{sp}}\right)^{T} \tilde{S}\left(\tilde{\mathbf{u}}-\tilde{\mathbf{u}}^{\mathrm{sp}}\right) \\
& \text { s.t. } \quad \tilde{\mathbf{y}}=\tilde{A} \Delta \tilde{\mathbf{u}}+\tilde{\mathbf{b}} \\
& \\
& \tilde{\mathbf{u}}=\tilde{\mathbf{u}}_{-1}+I_{L} \Delta \tilde{\mathbf{u}}
\end{aligned}
$$

where the vectors $\tilde{\mathbf{y}}, \tilde{\mathbf{u}}, \Delta \tilde{\mathbf{u}}, \tilde{\mathbf{y}}^{\mathrm{sp}}$ and $\Delta \tilde{\mathbf{u}}$ are composite vectors of the outputs, inputs, 
input changes, output setpoints and input set-points appearing in (4.20), defined as

$$
\tilde{\mathbf{y}}=\left[\begin{array}{c}
\mathbf{y}_{1} \\
\mathbf{y}_{2} \\
\vdots \\
\mathbf{y}_{p}
\end{array}\right] \quad \tilde{\mathbf{y}}^{\mathrm{sp}}=\left[\begin{array}{c}
\mathbf{y}_{1}^{\mathrm{sp}} \\
\mathbf{y}_{2}^{\mathrm{sp}} \\
\vdots \\
\mathbf{y}_{p}^{\mathrm{sp}}
\end{array}\right] \quad \tilde{\mathbf{u}}=\left[\begin{array}{c}
\mathbf{u}_{0} \\
\mathbf{u}_{1} \\
\vdots \\
\mathbf{u}_{m-1}
\end{array}\right] \quad \Delta \tilde{\mathbf{u}}=\left[\begin{array}{c}
\Delta \mathbf{u}_{0} \\
\Delta \mathbf{u}_{1} \\
\vdots \\
\Delta \mathbf{u}_{m-1}
\end{array}\right] \quad \tilde{\mathbf{u}}^{\mathrm{sp}}=\left[\begin{array}{c}
\mathbf{u}_{0}^{\mathrm{sp}} \\
\mathbf{u}_{1}^{\mathrm{sp}} \\
\vdots \\
\mathbf{u}_{m-1}^{\mathrm{sp}}
\end{array}\right]
$$

The mapping of the remaining parameters and matrices in 4.21 to those in 4.20 is given in Appendix A. Eliminating the equality constraints in 4.21) though substitution of $\tilde{\mathbf{y}}$ and $\tilde{\mathbf{u}}$ into the objective function, and setting the gradient to zero gives the optimal solution as

$$
\begin{aligned}
\Delta \tilde{\mathbf{u}} & =\left(\tilde{A}^{T} \tilde{Q} \tilde{A}+\tilde{R}+I_{L}^{T} \tilde{S} I_{L}\right)^{-1}\left[\tilde{A}^{T} \tilde{Q}\left(\tilde{\mathbf{y}}_{\mathbf{s p}}-\tilde{\mathbf{b}}\right)+I_{L}^{T} \tilde{S}\left(\tilde{\mathbf{u}}_{\mathbf{s p}}-\tilde{\mathbf{u}}_{-\mathbf{1}}\right)\right] \\
& =K_{1}\left(\tilde{\mathbf{y}}_{\mathbf{s p}}-\tilde{\mathbf{b}}\right)+K_{2}\left(\tilde{\mathbf{u}}_{\mathbf{s p}}-\tilde{\mathbf{u}}_{-\mathbf{1}}\right)
\end{aligned}
$$

consistent with the well-known solution to the unconstrained MPC problem as reported, for example, in Garcia and Morshedi [1986]. We note moreover, that (i) $K_{1}$ and $K_{2}$ can be computed ahead of time, and (ii) only the first $n_{u}$ rows of $K_{1}$ and $K_{2}$ are needed, where $n_{u}$ is the number of inputs, since it is only $\Delta \mathbf{u}_{0}$ that defines inputs that are applied to the DRTO plant model.

\subsection{Case Studies}

To execute the case studies below, MATLAB (version 2017a) is used to simulate the lower-level control implementation and real plant. The upper layer is formulated within AMPL (version 3.12.8) with the CONOPT solver (version 3.15C). The machine configuration is $3.40 \mathrm{GHz}$ Intel(R) Core(TM) i7-3770 processor and $8.00 \mathrm{~GB}$ of RAM 
with a Windows 8 64-bit operating system.

\subsubsection{Target tracking for a linear transfer function system}

\section{Performance analysis}

In this section, the performance of the approximation techniques is compared against the rigorous counterpart, with the performance of a decentralized configuration included to contrast this against that obtained with dynamic coordination via the closed-loop DRTO scheme.

Consider the transfer function for a copolymerization reactor, provided by Congalidis et al. [1986]:

$$
G(s)=\left[\begin{array}{ccccc}
\frac{0.34}{0.85 s+1} & \frac{0.21}{0.42 s+1} & \frac{0.50(0.50 s+1)}{0.12 s^{2}+0.40 s 1} & 0 & \frac{6.46(0.9 s+1)}{0.07 s^{2}+0.30 s+1} \\
\frac{-0.41}{2.41 s+1} & \frac{0.66}{1.51 s+1} & \frac{-0.3}{2.71 s+1} & 0 & \frac{-3.72}{0.80 s+1} \\
\frac{0.30}{2.54 s+1} & \frac{0.49}{1.54 s+1} & \frac{-0.71}{1.35 s+1} & \frac{-0.20}{2.71 s+1} & \frac{-4.71}{0.08 s^{2}+0.41 s+1} \\
0 & 0 & 0 & 0 & \frac{1.03(0.23 s+1)}{0.07 s^{2}+0.31 s+1}
\end{array}\right]
$$

The control structure for this case study has been studied by Congalidis et al. [1986]; Gudi and Rawlings 2006]. Congalidis et al. [1986] propose a decentralized approach for this transfer function system with three pairing sets based on an RGA analysis. Gudi and Rawlings [2006] consider three control loops, the first of which is a multivariable loop comprising inputs $u_{2}$ and $u_{3}$, and outputs $y_{1}$ and $y_{2}$, the second comprising $u_{4}$ and $y_{3}$, and the third comprising $u_{5}$ and $y_{4}$. The first input is kept constant, as suggested by Congalidis et al. [1986]. In this work, the plant transfer function is partitioned into two subsystems in which the second and third loops considered by Gudi and Rawlings 2006 are combined into one subsystem. In addition, the first input is allowed to vary to demonstrate the MPC's capability of handling 
a non-square system. Thus, the first subsystem comprises the first two outputs and first three inputs. The complete transfer function model 4.24 is used to generate the full plant response and is embedded in the primary optimization problem in the DRTO formulations. The two subsystems are used for the lower level MPCs to generate control actions and MPC optimization subproblems in the DRTO formulation, given by

$$
\begin{gathered}
G_{1}(s)=\left[\begin{array}{ccc}
\frac{0.34}{0.85 s+1} & \frac{0.21}{0.42 s+1} & \frac{0.50(0.50 s+1)}{0.12 s^{2}+0.40 s 1} \\
\frac{-0.41}{2.41 s+1} & \frac{0.66}{1.51 s+1} & \frac{-0.3}{2.71 s+1}
\end{array}\right] \\
G_{2}(s)=\left[\begin{array}{cc}
\frac{-0.20}{2.71 s+1} & \frac{-4.71}{0.08 s^{2}+0.41 s+1} \\
0 & \frac{1.03(0.23 s+1)}{0.07 s^{2}+0.31 s+1}
\end{array}\right]
\end{gathered}
$$

By partitioning the transfer function system in this fashion, each subsystem is assumed to have knowledge of only the portion of the plant assigned to it. Since the first subsystem is a non-square system, an input set-point trajectory can be assigned to the MPC along with two output set-point trajectories.

The objective function for target tracking with associated input and output constraints is defined as:

$$
\begin{aligned}
\min _{\mathrm{y}^{\mathrm{ref}}} & \Delta t_{\mathrm{MPC}} \sum_{j=1}^{N}\left(y_{j, 1}^{\mathrm{DRTO}}-y_{1}^{\mathrm{target}}\right)^{2}+\left(y_{j, 2}^{\mathrm{DRTO}}-y_{2}^{\text {target }}\right)^{2} \\
& +\left(y_{j, 3}^{\mathrm{DRTO}}-y_{3}^{\mathrm{target}}\right)^{2}+\left(y_{j, 4}^{\mathrm{DRTO}}-y_{4}^{\text {target }}\right)^{2}+\left(u_{j-1,3}^{\mathrm{DRTO}}-u_{3}^{\text {target }}\right)^{2} \\
& -2 \leq y_{j, i}^{\mathrm{DRTO}} \leq 2, \quad i=1, \ldots, 4 \quad j=1, \ldots, N \\
& -2 \leq y_{j, i}^{\mathrm{ref}} \leq 2, \quad i=1, \ldots, 4 \quad j=1, \ldots, N \\
& -2 \leq u_{j, i} \leq 20, \quad i=1, \ldots, 5, \quad j=0, \ldots, N-1
\end{aligned}
$$

where $y_{j, i}^{\text {DRTO }}$ and $y_{j, i}^{\text {ref }}$ are the $i$ th component of $\mathbf{y}_{j}^{\text {DRTO }}$ and $\mathbf{y}_{j}^{\text {ref }}$ respectively, and 
Table 4.1: Design parameters for the DRTO and MPC layers for the linear transfer function system

\begin{tabular}{lll}
\hline Parameter & Description & Value \\
\hline$T_{\text {sim }}$ & total simulation time & 10 \\
$\Delta t_{D R T O}$ & DRTO sample time & 2 \\
$\Delta t_{M P C}$ & MPC sample time & 1 \\
$p$ & prediction horizon & 15 \\
$m$ & control horizon & 10 \\
$Q$ & output tracking weight & MPC1:diag $(20,20)$ \\
& & MPC2: $\operatorname{diag}(20,20)$ \\
$R$ & move suppression weight & MPC1:diag $(2,2,2)$ \\
$S$ & & MPC2:diag $(2,2)$ \\
& control tracking weight & MPC1:diag $(0,0,1)$ \\
$N$ & & MPC2: $\operatorname{diag}(0,0)$ \\
$n$ & optimization horizon & 20 \\
$\rho$ & closed-loop prediction horizon for hybrid case & 4 \\
\hline
\end{tabular}

$u_{j, i}$ represents the $i$ th input corresponding to DRTO step $j$, with the constraints applied in accordance with the DRTO scheme used. The set-point targets are assigned as $y_{1}^{\text {target }}=1, y_{2}^{\text {target }}=1, y_{3}^{\text {target }}=1, y_{4}^{\text {target }}=0, u_{3}^{\text {target }}=0.5$. The targets are kept constant throughout the set-point trajectories for the decentralized MPCs. The parameters for setting up the DRTO and MPC layers for the distributed cases are summarized in Table 4.1. The performance for each case is evaluated by using the sum of squared errors (SSE) for all inputs and output with assigned set-point trajectories.

The performance evaluated numerically is presented in Table 4.2. The SSE value for the rigorous formulation with the input clipping method is only slightly larger than the case without the input clipping method, but has a significantly shorter computing time. This is because the number of constraints drops markedly when the input constraints are enforced only for the first control inputs generated by the MPC optimization subproblems by the input saturation mechanism, which also leads to a 
Table 4.2: Summary of performance for the linear transfer function system

\begin{tabular}{lllll}
\hline Control architecture & Solution strategy & SSE & No. of variables & $\begin{array}{l}\text { Avg. } \\
\text { DRTO } \\
\text { comp. } \\
\text { time (sec) }\end{array}$ \\
& & & & 39.60 \\
& & & & 10.02 \\
Rigorous & KKT & 3.0410 & 21124 & 2.63 \\
Rigorous w / clipping & KKT & 3.0548 & 17524 & 2.60 \\
Hybrid & Analytical & 3.0548 & 8857 & 1.88 \\
Hybrid w/ clipping & KKT & 3.0848 & 6003 & 0.86 \\
Hybrid w/ clipping & Analytical & 3.1058 & 5283 & $\mathrm{n} / \mathrm{a}$ \\
decentralized MPCs & n/a & 3.1058 & 3158 & $\mathrm{n} / \mathrm{a}$ \\
\hline
\end{tabular}

reduction of problem size. The responses for the rigorous formulation are shown in Figures 4.3 and 4.4 .

The three cases with the hybrid formulation exhibit significant improvement in the computational speed, but with slightly worse performance when compared to the corresponding rigorous formulations with the same implementation variation, because only a limited number of rigorous predictions are incorporated and an open-loop prediction of plant response is used for the rest of DRTO prediction horizon. The results for the hybrid formulation are shown in Figures 4.5 and 4.6 .

When compared to the decentralized configuration shown in Table 4.2, all the DRTO formulations demonstrate superior performance in terms of lower SSE values, which further confirms the efficacy of using DRTO formulations to judiciously assign setpoint trajectories based on the closed-loop prediction of the plant response that accounts for interactions among controllers and the plant. 

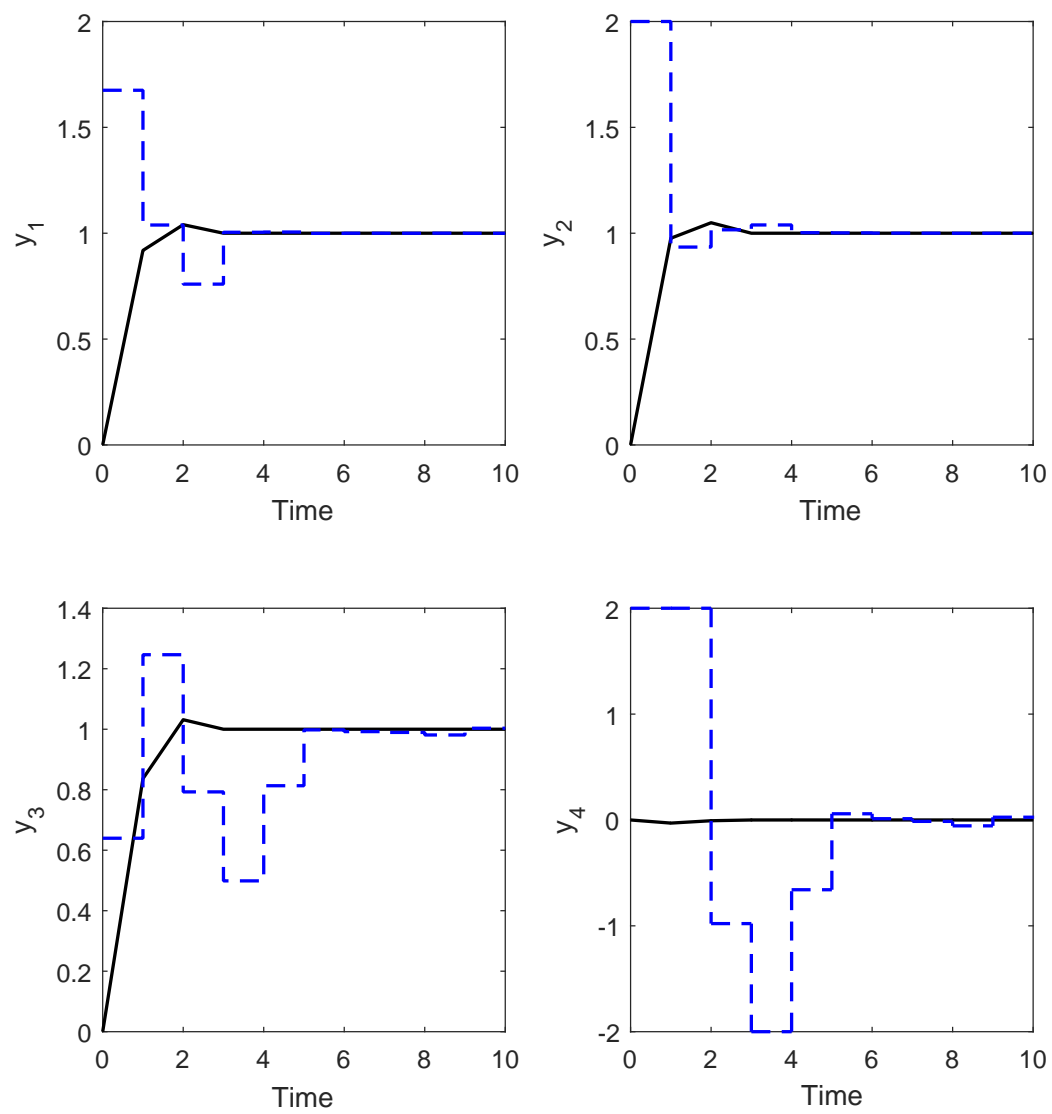

Figure 4.3: Output responses with set-point trajectories for the rigorous case 

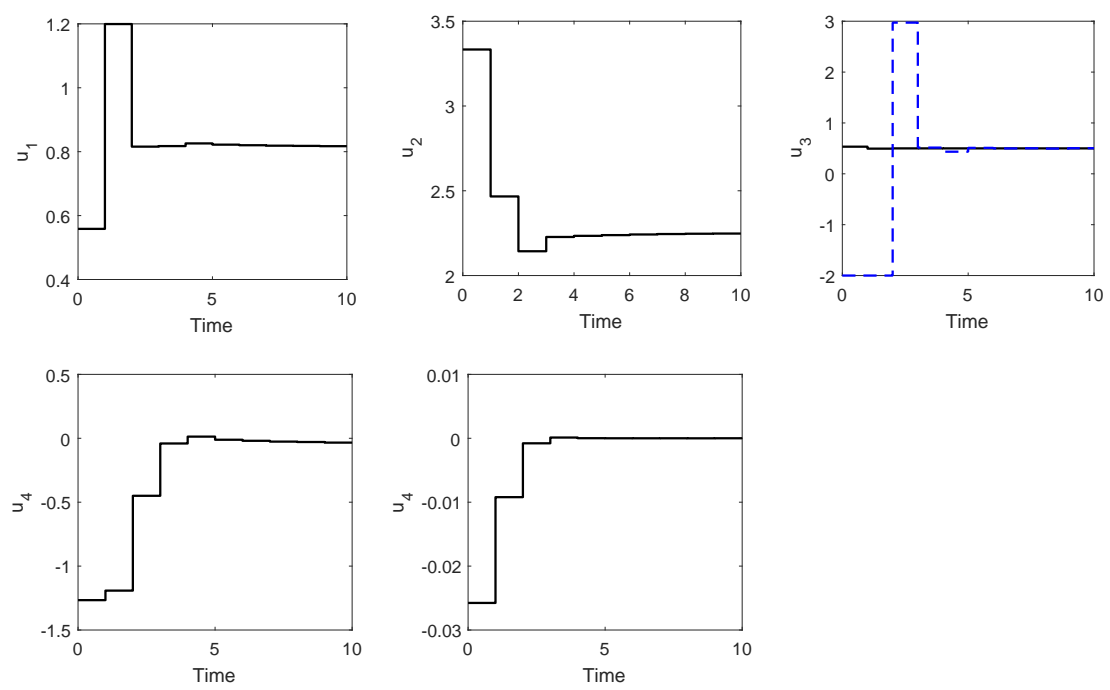

Figure 4.4: Input responses with set-point trajectories for the rigorous case

\section{Problem size analysis}

As the number of MPC optimization subproblems included in the closed-loop simulation and their associated length of control horizon increases, the rigorous formulation solution time can potentially be intractable. Those two causes can be addressed either separately or simultaneously. Figure 4.7 shows the growth of problem sizes for DRTO formulations due to the increment on control horizons of the MPC optimization subproblems. The prediction horizon $p$ is set to 35 consistently to accommodate a large control horizon. The other parameters are left consistent with the ones from the previous section.

First, the significant gap between the rigorous formulation and hybrid formulation can be attributed to the reduction in the number of MPC optimization subproblems in the hybrid formulation, since the last MPC optimization subproblem in the hybrid formulation approximates the closed-loop behaviour for a large portion of the closedloop prediction horizon, while the rigorous case generates a set of control inputs at every step of the DRTO horizon. 

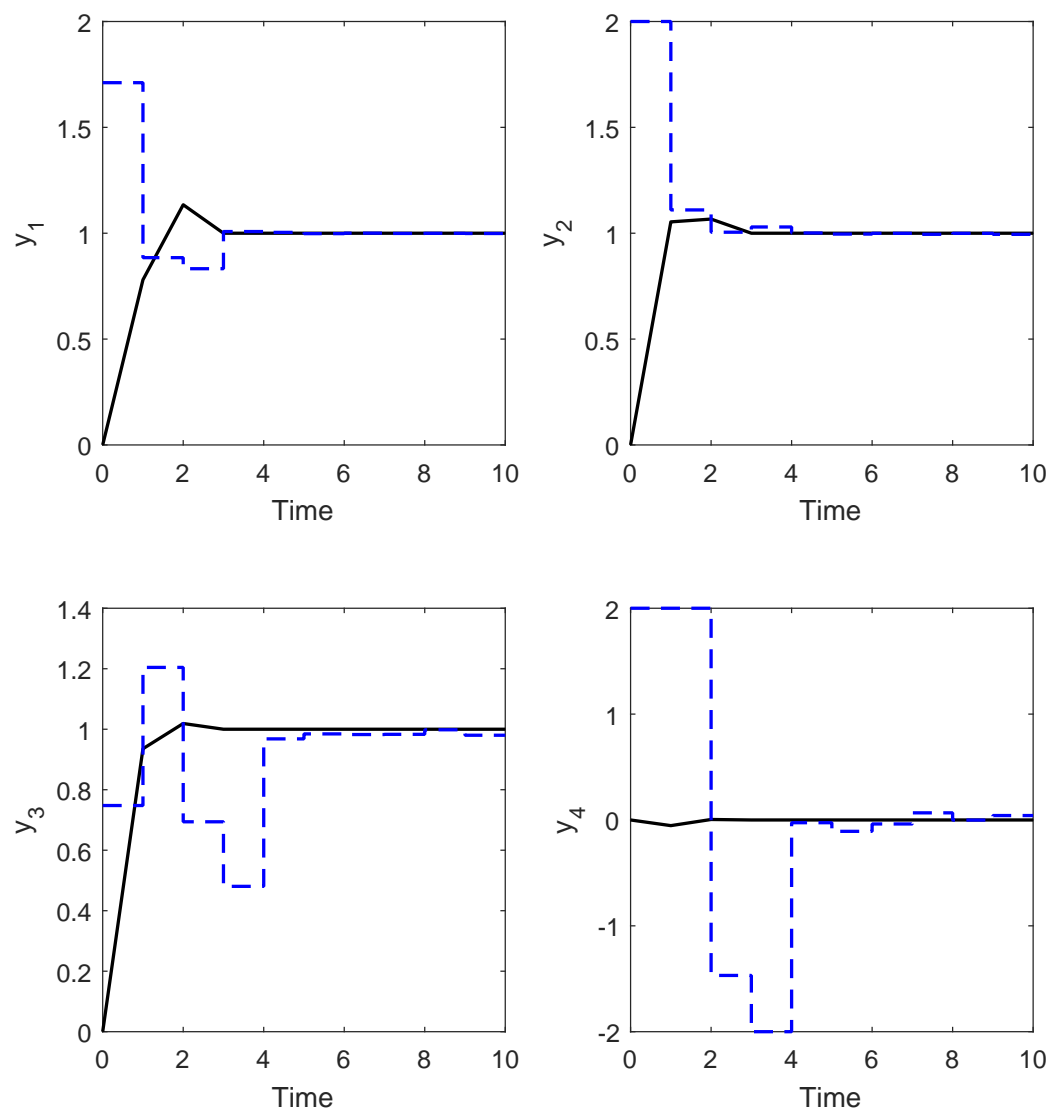

Figure 4.5: Output responses with set-point trajectories for the hybrid case 

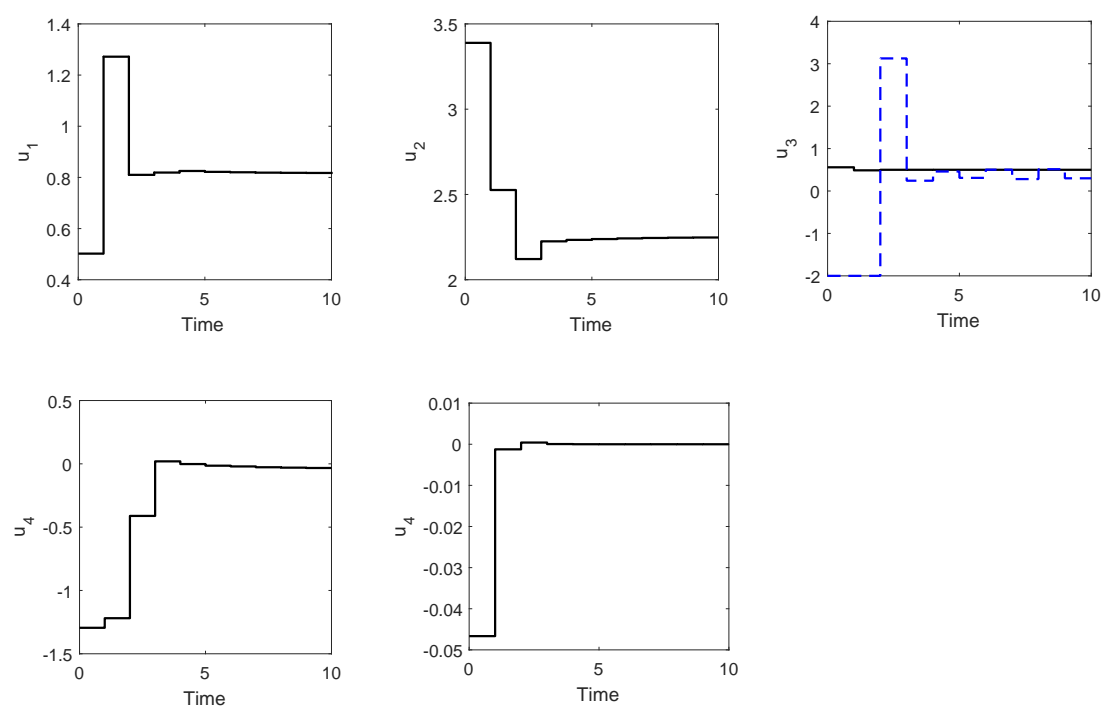

Figure 4.6: Input responses with set-point trajectories for the hybrid case

Second, for the cases with the rigorous formulation, when the control horizon is small, there is no noticeable differences between the formulations with the input clipping method and those without. This is due to the limited number of input constraints that are eliminated. The input clipping method applies the input constraints only to the first control move of each MPC subproblem over the DRTO horizon. This is the reason why the improvement becomes significant when control horizons are long. The reduction in the number of variables through the analytical solution of the unconstrained MPC subproblems as opposed to the KKT formulation, as discussed in section ??, is evident in Figure 4.7 .

Lastly, for the cases with the hybrid formulation, the input clipping method does not substantially decrease the problem size anymore, since a large portion of the closedloop prediction horizon is approximated by the last MPC optimization subproblem. This MPC optimization subproblem computes the control inputs for the control horizon of $n$ to $N-n-1$ and applies the entire horizon of inputs to the dynamic plant to generate the plant response. Thus, only the closed-loop prediction approximation 


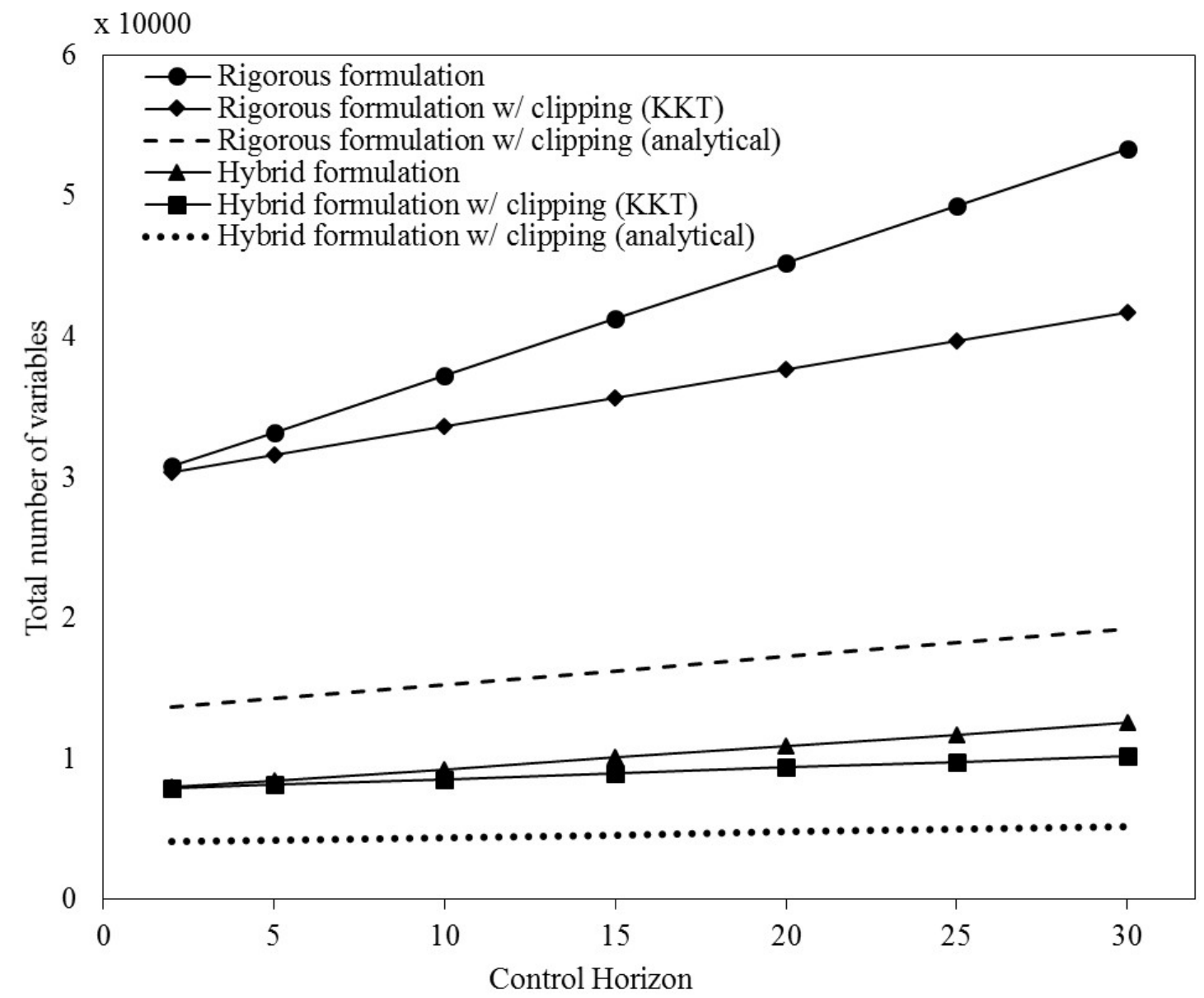

Figure 4.7: The problem sizes with increasing control horizon

portion of the hybrid formulation benefits from the clipping method.

Since the hybrid formulation contains a small number of MPC optimization subproblems to generate the closed-loop response, followed by an MPC optimization subproblem that generates input trajectories that are directly implemented, the length of closed-loop prediction becomes an additional degree of freedom that needs to be determined a priori. Figure 4.8 demonstrates the effect of the closed-loop prediction length. The entire DRTO prediction horizon is set to be 40 and other parameters are consistent with those used in the previous section. It can be clearly seen that as 


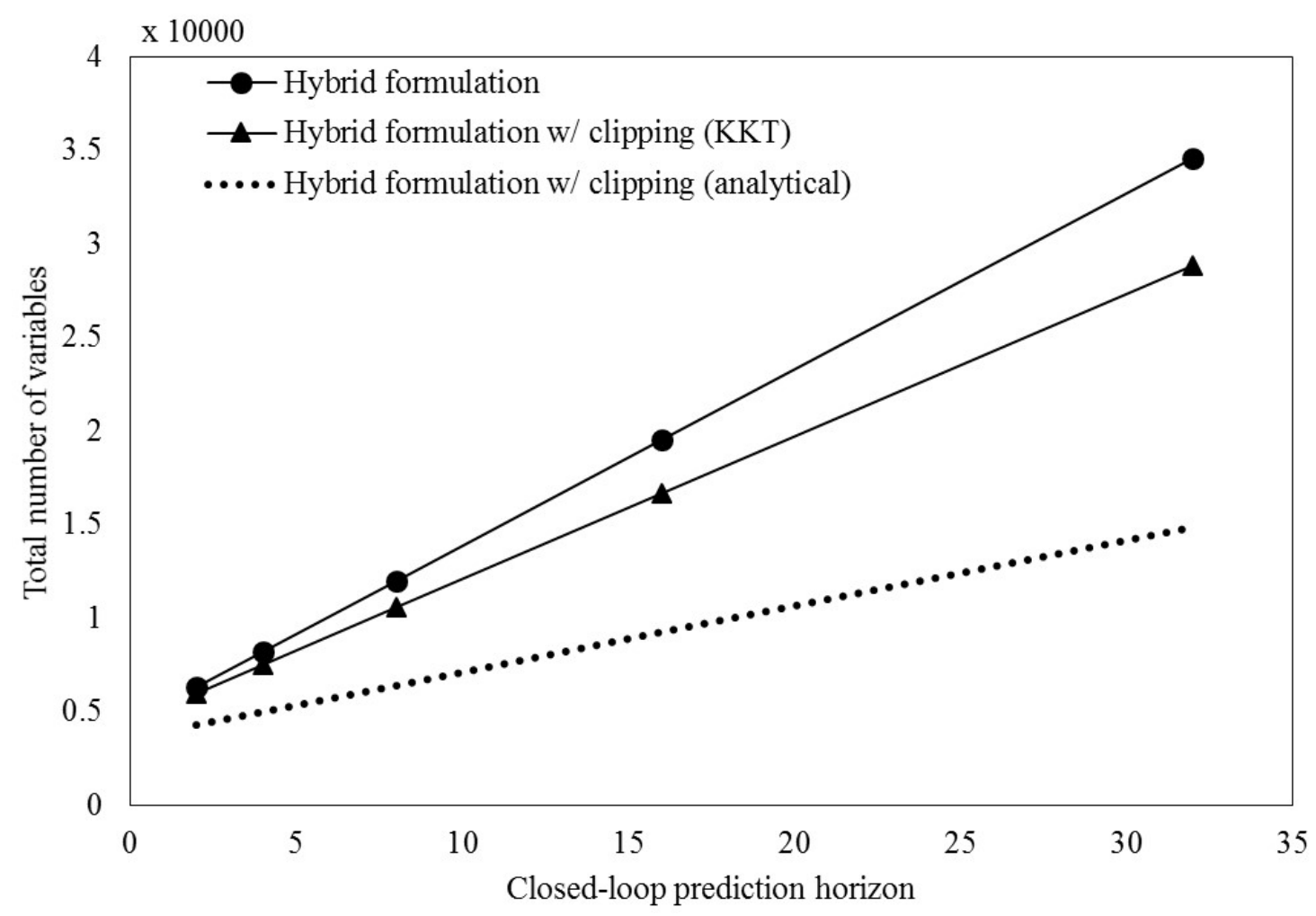

Figure 4.8: The problem sizes for hybrid formulation with increasing closed-loop prediction horizon

the length of closed-loop prediction increases, the problem size for hybrid formulation grows substantially due to the increasing number of MPC optimization subproblems needed to generate the closed-loop prediction.

\subsubsection{Dynamic optimization of a nonlinear system of two CSTRs in series}

In this section, the effect of coordination using the DRTO formulation is investigated in the context of economic optimization. By assigning calculated set-point trajectories, the DRTO formulation is able to find the economic optimum based on the process conditions and economic objective function. In order to achieve this, the DRTO for- 


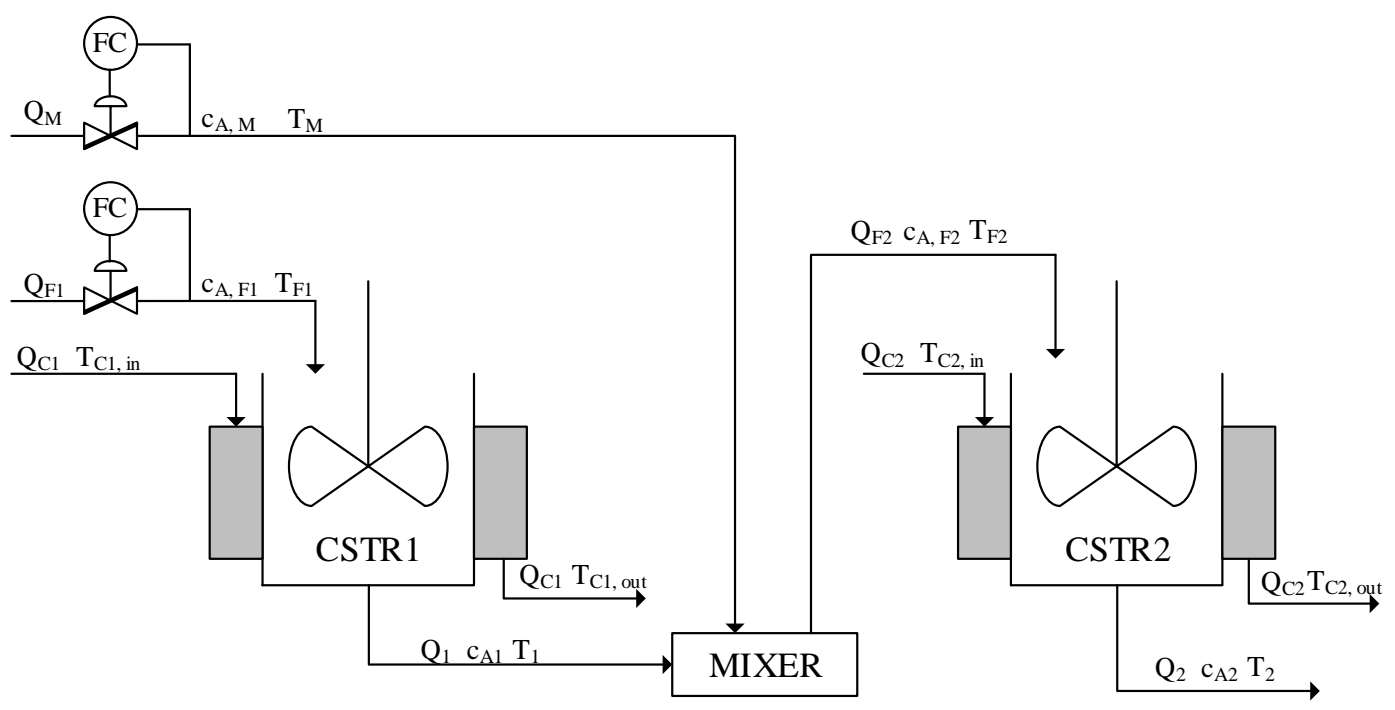

Figure 4.9: Schematics of the two CSTRs in series

mulation is able to utilize the closed-loop prediction of the controller actions along with the process dynamics, to generate time-varying set-points at the current time instance.

We consider the scenario of two CSTR in series, using the model provided by Loeblein and Perkins 1998 with schematic shown in Figure 4.9. The reaction $A \rightarrow B$ occurs in both CSTRs, but a side reaction $B \rightarrow C$ also occurs during the process. Pure reactant $\mathrm{A}$ enters the systems in the feed stream $F 1$ for the first CSTR and the intermediate feed stream $M$ to the mixer. The product from the first reactor along with the intermediate fresh feed of reactant $\mathrm{A}$ is fed to the second reactor to further continue the reaction. Cooling jackets are installed to both reactors to avoid overheating. Constant liquid density and heat capacity are assumed. The levels in both CSTRs are also assumed to be perfectly controlled.

The rigorous model for the process is constructed with the following mass and energy 
balances:

$$
\begin{aligned}
\frac{d c_{A 1}}{d t} & =Q_{F 1} c_{A, F 1}-Q_{1} c_{A 1}-c_{A 1} V_{1} k_{0, I} e^{\left(-\frac{E_{I}}{R T_{1}}\right)} \\
\frac{d c_{B 1}}{d t} & =Q_{F 1} c_{B, F 1}-Q_{1} c_{B 1}+c_{A 1} V_{1} k_{0, I} e^{\left(-\frac{E_{I}}{R T_{1}}\right)}-c_{B 1} V_{1} k_{0, I I} e^{-\frac{E_{I I}}{R T_{1}}} \\
\frac{d c_{C 1}}{d t} & =Q_{F 1} c_{C, F 1}-Q_{1} c_{C 1}+c_{B 1} V_{1} k_{0, I I} e^{\left(-\frac{E_{I I}}{R T_{1}}\right)} \\
\frac{d T_{1}}{d t} & =Q_{F 1} T_{F 1}-Q_{1} T_{1}-\frac{\Delta H_{R, I}}{\rho c_{p}} c_{A 1} V_{1} k_{0, I} e^{\left(-\frac{E_{I}}{R T_{1}}\right)}-\frac{\Delta H_{R, I I}}{\rho c_{p}} c_{B 1} V_{1} k_{0, I I} e^{\left(-\frac{E_{I I}}{R T_{1}}\right)}-q_{\text {cool }, 1} \\
\frac{d c_{A 2}}{d t} & =Q_{F 2} c_{A, F 2}-Q_{2} c_{A 2}-c_{A 2} V_{2} k_{0, I} e^{\left(-\frac{E_{I}}{R T_{2}}\right)} \\
\frac{d c_{B 2}}{d t} & =Q_{F 2} c_{B, F 2}-Q_{2} c_{B 2}+c_{A 2} V_{2} k_{0, I} e^{\left(-\frac{E_{I}}{R T_{2}}\right)}-c_{B 2} V_{2} k_{0, I I} e^{-\frac{E_{I I}}{R T_{2}}} \\
\frac{d c_{C 2}}{d t} & =Q_{F 2} c_{C, F 2}-Q_{2} c_{C 2}+c_{B 2} V_{2} k_{0, I I} e^{\left(-\frac{E_{I I}}{R T_{2}}\right)} \\
\frac{d T_{2}}{d t} & =Q_{F 2} T_{F 2}-Q_{2} T_{2}-\frac{\Delta H_{R, I}}{\rho c_{p}} c_{A 2} V_{2} k_{0, I} e^{\left(-\frac{E_{I}}{R T_{2}}\right)}-\frac{\Delta H_{R, I I}}{\rho c_{p}} c_{B 2} V_{2} k_{0, I I} e^{\left(-\frac{E_{I I}}{R T_{2}}\right)}-q_{\text {cool }, 2}
\end{aligned}
$$

With the following algebraic equations:

$$
\begin{aligned}
q_{c o o l, i} & =U_{a, i} \Delta T_{l n, i} \quad i=1,2 \\
\Delta T_{l n, i} & =\frac{T_{c i, \mathrm{out}}-T_{c i, \mathrm{in}}}{\ln \left(T_{i}-T_{c i, \mathrm{in}}\right) /\left(T_{i}-T_{c i, \mathrm{out}}\right)} \quad i=1,2 \\
q_{c o o l, i} & =Q_{c, i}\left(T_{c i, \mathrm{out}}-T_{c i, \mathrm{in}}\right) \quad i=1,2 \\
Q_{F 2} & =Q_{1}+Q_{M} \\
Q_{F 2} c_{i, F 2} & =Q_{1} c_{i, 1}+Q_{M} c_{i, M} \quad i=A, B, C \\
Q_{F 2} T_{F 2} & =Q_{1} T_{1}+Q_{M} T_{M}
\end{aligned}
$$

The following constraints are applied to ensure that product quality, safety, supply, 
and operational constraints are met:

$$
\begin{array}{r}
T_{1} \leq 350 \mathrm{~K}, \quad T_{2} \leq 350 \mathrm{~K} \\
Q_{F 1}+Q_{M} \leq 0.8 \mathrm{~m}^{3} / \mathrm{s} \\
T_{c 1, \text { out }} \leq 330 \mathrm{~K}, \quad T_{c 2, \text { out }} \leq 300 \mathrm{~K} \\
Q_{F 1} \geq 0.05 \mathrm{~m}^{3} / \mathrm{s}, \quad Q_{M} \geq 0.05 \mathrm{~m}^{3} / \mathrm{s} \\
c_{A 2} \leq 0.3 \mathrm{kmol} / \mathrm{m}^{3}
\end{array}
$$

The economic objective function is also provided by Loeblein and Perkins 1998 and expressed on a per unit time basis to maximize the profit calculated based on revenue, and material and utility cost:

$$
\begin{array}{r}
\phi=10\left(Q_{F 1} c_{A, F 1}+Q_{M} c_{A, M}-Q_{2}\left(c_{A, 2}+c_{C, 2}\right)\right) \\
-0.01 q_{c o o l, 1}-q_{c o o l, 2}-0.1 Q_{F 1}-0.1 Q_{M}
\end{array}
$$

The objective function is integrated in the DRTO formulation to account for the accumulated profit throughout the transition.

The overall process is partitioned into two subsystems comprising the first and second reactors respectively, with the reactor temperatures selected at the outputs, and $Q_{F 1}$ and $Q_{M}$ as the inputs.

A complete listing of the process parameters and their values is given in Appendix B. The plant is regulated by all DRTO formulations introduced with different combinations of approximation techniques described in this paper. The design parameters for all DRTO formulations are summarized in Table 4.3 and all initial values are summarized in Table 4.4. The accumulated profits, along with problem sizes solved by the DRTO formulations and average computing time are summarized in Table 4.5. 
Table 4.3: Design parameters for the DRTO and MPC layers for the two-CSTR system

\begin{tabular}{lll}
\hline Parameter & Description & Value \\
\hline$T_{\text {sim }}$ & total simulation time & $10 \mathrm{~s}$ \\
$\Delta t_{M P C}$ & MPC sample time & $0.5 \mathrm{~s}$ \\
$\Delta t_{D R T O}$ & DRTO sample time & $2 \mathrm{~s}$ \\
$p$ & prediction horizon & 11 \\
$m$ & control horizon & 10 \\
$N$ & optimization horizon & 20 \\
$n$ & closed-loop prediction horizon for & 8 \\
& hybrid case & \\
$\rho$ & penalty parameter & 100 \\
$Q$ & output tracking weight & MPC1:10 \\
& & MPC2: 1 \\
$R$ & move suppression weight & MPC1:10 \\
& & MPC2:10 \\
$S$ & control tracking weight & MPC1:0 \\
& & MPC2: 0 \\
$\left.\mathbf{y}_{\text {min }}^{\text {sp }} \mathbf{y}_{\text {max }}^{\text {sp }}\right]$ & bounds for set-point trajectories & MPC1:[100 360] \\
& & MPC2:[100 360] \\
\hline
\end{tabular}

Table 4.4: Initial steady-state values for inputs and outputs of the integrated plant.

\begin{tabular}{cccccc}
\hline variable & value & variable & value & variable & value \\
\hline$c_{A 1}$ & $0.1217 \mathrm{kmol} / \mathrm{m}^{3}$ & $c_{A 2}$ & $0.2106 \mathrm{kmol} / \mathrm{m}^{3}$ & $Q_{F 1}$ & $0.2 \mathrm{~m}^{3} / \mathrm{s}$ \\
$c_{B 1}$ & $19.7252 \mathrm{kmol} / \mathrm{m}^{3}$ & $c_{B 2}$ & $19.6627 \mathrm{kmol} / \mathrm{m}^{3}$ & $Q_{M}$ & $0.2 \mathrm{~m}^{3} / \mathrm{s}$ \\
$c_{C 1}$ & $0.1530 \mathrm{kmol} / \mathrm{m}^{3}$ & $c_{C 2}$ & $0.1268 \mathrm{kmol} / \mathrm{m}^{3}$ & & \\
$T_{1}$ & $342.1333 \mathrm{~K}$ & $T_{2}$ & $331.598 \mathrm{~K}$ & & \\
\hline
\end{tabular}


Table 4.5: The process results for the two-CSTR system

\begin{tabular}{|c|c|c|c|c|}
\hline Control architecture & $\begin{array}{l}\text { Solution } \\
\text { strategy }\end{array}$ & $\begin{array}{l}\text { Accumu } \\
\text { profits }\end{array}$ & No. of variables & $\begin{array}{l}\text { Avg. } \\
\text { DRTO } \\
\text { computing } \\
\text { time (sec) }\end{array}$ \\
\hline Rigorous & KKT & 892.758 & 8508 & 1.78 \\
\hline Rigorous w/ clipping & $\mathrm{KKT}$ & 892.229 & 7068 & 0.89 \\
\hline Rigorous w/ clipping & Analytical & 892.229 & 3546 & 0.44 \\
\hline Hybrid & $\mathrm{KKT}$ & 892.748 & 3790 & 0.56 \\
\hline Hybrid w/ clipping & $\mathrm{KKT}$ & 892.196 & 3358 & 0.39 \\
\hline Hybrid w/ clipping & Analytical & 892.196 & 1988 & 0.14 \\
\hline
\end{tabular}

The optimal process conditions achieved are listed as followed: $Q_{\mathrm{F} 1}=0.274 \mathrm{~m}^{3} / \mathrm{s}$, $Q_{\mathrm{M}}=0.236 \mathrm{~m}^{3} / \mathrm{s}, T_{1}=350 \mathrm{~K}, T_{\mathrm{c} 2 \text {,out }}=300 \mathrm{~K}, \phi=82.84 £ / \mathrm{h}$. These values are consistent with those provided by Loeblein and Perkins [1998 without specifying the optimum condition in advance.

Results for the rigorous and approximate DRTO formulations are presented in Table 4.5. It can be seen that the performance of the cases with approximation techniques applied can decrease the computational time with only a slight performance drop, when compared with that of the rigorous case. By combining different solution strategies and approximation techniques, the problem sizes can be substantially decreased. Further reductions could be expected for larger control horizons. However, since the problem size analysis was done in the previous section, this case study is primarily intended to show the impact of the closed-loop approximation on the economic performance in addition to the computational performance, and also how the approximation fares with application to a nonlinear plant. The plant responses for the hybrid formulation with clipping are shown in Figures 4.10 and 4.11 . 

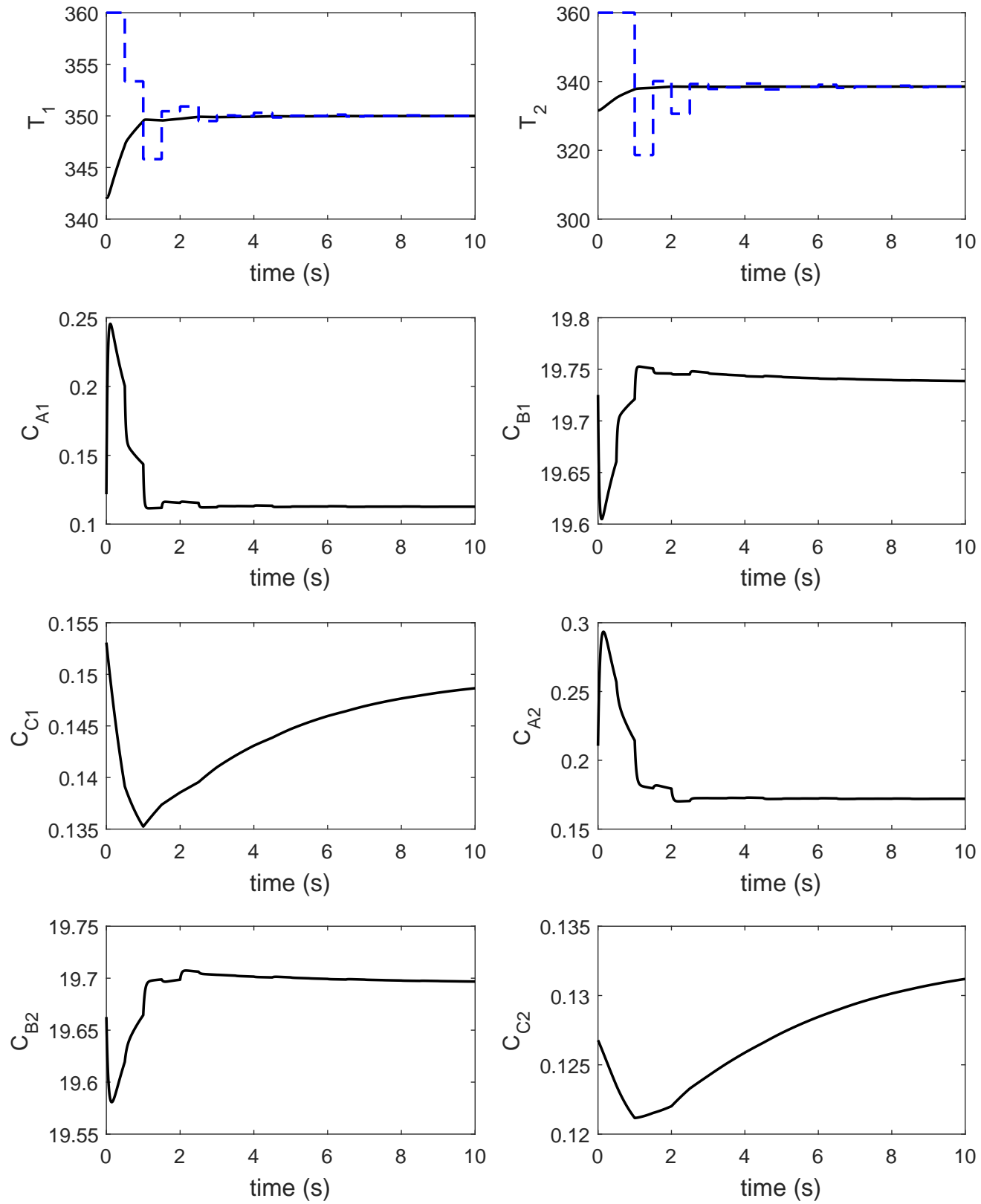

Figure 4.10: The output responses with setpoint trajectories for the hybrid with clipping case 

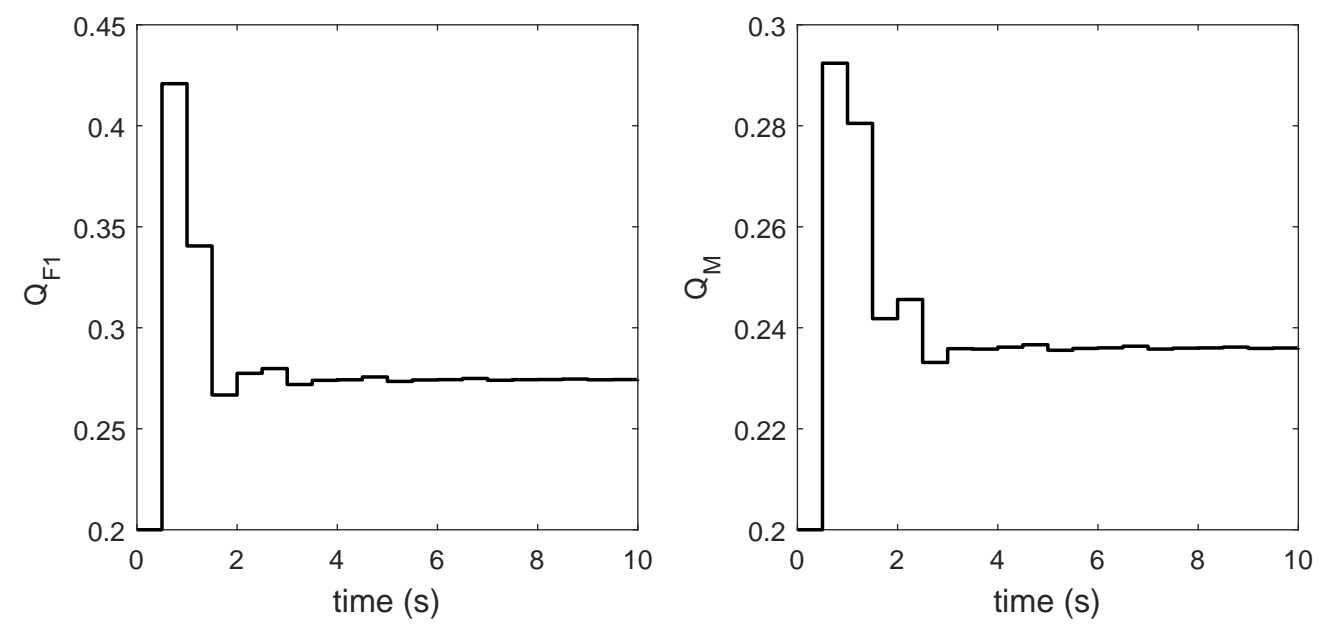

Figure 4.11: The input responses with setpoint trajectories for the hybrid with clipping case

\section{Appendix A: Mapping of Parameters Between MPC Representations}

The parameter vectors and matrices in the MPC formulation 4.21) are related to those in MPC formulation 4.20 as follows, for a dynamic system with $n_{u}$ inputs, $n_{y}$ outputs and $n_{x}$ states, and MPC prediction horizon $p$ and control horizon $m$.

The symmetric positive semidefinite matrices $\tilde{Q} \in R^{p n_{y} \times p n_{y}}, \tilde{R} \in R^{m n_{u} \times m n_{u}}$, and $\tilde{S} \in R^{m n_{u} \times m n_{u}}$ in the objective function are block diagonal matrices comprised of the weighting matrices $Q \in R^{n_{y} \times n_{y}}, R \in R^{n_{u} \times n_{u}}$, and $S \in R^{n_{u} \times n_{u}}$ as follows,

$$
\tilde{Q}=\left[\begin{array}{cccc}
Q & 0 & \ldots & 0 \\
0 & Q & \ldots & 0 \\
\vdots & \vdots & \ddots & \vdots \\
0 & 0 & \ldots & Q
\end{array}\right] \quad \tilde{R}=\left[\begin{array}{cccc}
R & 0 & \ldots & 0 \\
0 & R & \ldots & 0 \\
\vdots & \vdots & \ddots & \vdots \\
0 & 0 & \ldots & R
\end{array}\right] \quad \tilde{S}=\left[\begin{array}{cccc}
S & 0 & \ldots & 0 \\
0 & S & \ldots & 0 \\
\vdots & \vdots & \ddots & \vdots \\
0 & 0 & \ldots & S
\end{array}\right]
$$

$I_{L} \in R^{m n_{u} \times m n_{u}}$ relates future inputs to future input changes over the control horizon 
$m$, where $I \in R^{n_{u} \times n_{u}}$ within $I_{L}$ represents the identity matrix of dimension $n_{u}$ :

$$
I_{L}=\left[\begin{array}{cccc}
I & 0 & \ldots & 0 \\
I & I & \ldots & 0 \\
\vdots & \vdots & \ddots & \vdots \\
I & I & \ldots & I
\end{array}\right]
$$

$\tilde{\mathbf{u}}_{-1}$ is a composite vector of the inputs most recently applied to the plant, $\mathbf{u}_{-1}$,

$$
\tilde{\mathbf{u}}_{-1}=\left[\mathbf{u}_{-1}^{T} \ldots \mathbf{u}_{-1}^{T}\right]^{T}
$$

The dynamic matrix $\tilde{A}$ used to relate the predicted outputs to future input changes is constructed from the linear dynamic system matrices $A, B$ and $C$ via

$$
\tilde{A}=\left[\begin{array}{ccc}
C B & \ldots & 0 \\
\vdots & \ddots & \vdots \\
\sum_{i=0}^{m-1} C A^{i} B & \ldots & C B \\
\sum_{i=0}^{m} C A^{i} B & \ldots & C A B+C B \\
\vdots & \ddots & \vdots \\
\sum_{i=0}^{p-1} C A^{i} B & \ldots & \sum_{i=0}^{p-m} C A^{i} B
\end{array}\right]
$$


with $\tilde{\mathbf{b}}$ in 4.21 defined as

$$
\tilde{\mathbf{b}}=\left[\begin{array}{c}
C A \\
\vdots \\
C A^{m} \\
C A^{m+1} \\
\vdots \\
C A^{p}
\end{array}\right] \mathbf{x}_{0}+\left[\begin{array}{c}
C B \\
\vdots \\
\sum_{i=0}^{m-1} C A^{i} B \\
\vdots \\
\sum_{i=0}^{m-1} C A^{i} B
\end{array}\right] \mathbf{u}_{-1}+\left[\begin{array}{c}
\mathbf{d}_{k+1} \\
\vdots \\
\mathbf{d}_{k+m} \\
\mathbf{d}_{k+m+1} \\
\vdots \\
\mathbf{d}_{k+p}
\end{array}\right]
$$

where $\mathbf{x}_{0} \in R^{n_{x}}$ is the initial state, and $\mathbf{u}_{-1} \in R^{n_{u}}$ is the vector of inputs implemented at the previous step.

\title{
Appendix B: Case Study 2 Parameters
}

\author{
Symbol Description and steady-state values \\ $c_{A, F 1} \quad$ concentration of $\mathrm{A}$ in feed to first reactor, $20 \mathrm{kmol} / \mathrm{m}^{3}$ \\ $c_{B, F 1} \quad$ concentration of $\mathrm{B}$ in feed to first reactor, $0 \mathrm{kmol} / \mathrm{m}^{3}$ \\ $c_{C, F 1} \quad$ concentration of $\mathrm{C}$ in feed to first reactor, $0 \mathrm{kmol} / \mathrm{m}^{3}$ \\ $c_{A, M} \quad$ concentration of $\mathrm{A}$ in feed to mixer, $20 \mathrm{kmol} / \mathrm{m}^{3}$ \\ $c_{B, M} \quad$ concentration of $\mathrm{B}$ in feed to mixer, $0 \mathrm{kmol} / \mathrm{m}^{3}$ \\ $c_{C, M} \quad$ concentration of $\mathrm{C}$ in feed to mixer, $0 \mathrm{kmol} / \mathrm{m}^{3}$ \\ $E_{I} / R \quad$ activation energy of main reaction, $6000 \mathrm{~K}$ \\ $E_{I I} / R \quad$ activation energy of side reaction, $4500 \mathrm{~K}$ \\ $k_{0, I} \quad$ main reaction rate constant, $2.7 \times 10^{8} \mathrm{~s}^{-1}$ \\ $k_{0, I I} \quad$ side reaction rate constant, $160 \mathrm{~s}^{-1}$

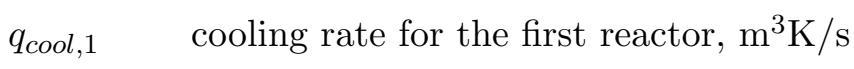 \\ $q_{c o o l, 2} \quad$ cooling rate for the second reactor, $\mathrm{m}^{3} \mathrm{~K} / \mathrm{s}$ \\ $Q_{c 1} \quad$ cooling water flow to first reactor, $0.7 \mathrm{~m}^{3} / \mathrm{s}$
}


$Q_{c 2} \quad$ cooling water flow to second reactor, $0.7 \mathrm{~m}^{3} / \mathrm{s}$

$T_{c 1, i n} \quad$ inlet cooling water temperature to first reactor, $300 \mathrm{~K}$

$T_{c 2, \text { in }} \quad$ inlet cooling water temperature to second reactor, $275 \mathrm{~K}$

$T_{F 1} \quad$ temperature of feed flow to the first reactor, $300 \mathrm{~K}$

$T_{M} \quad$ temperature of feed flow to the mixer, $300 \mathrm{~K}$

$U_{a 1} \quad$ heat transfer coefficient in the first reactor, $0.35 \mathrm{~m}^{3} / \mathrm{s}$

$U_{a 2} \quad$ heat transfer coefficient in the second reactor, $0.35 \mathrm{~m}^{3} / \mathrm{s}$

$V_{1} \quad$ holdup in the first reactor, $5 \mathrm{~m}^{3}$

$V_{2} \quad$ holdup in the second reactor, $5 \mathrm{~m}^{3}$ 


\section{Chapter 5}

\section{Inclusion of Nonlinearity}

In previous chapters, the DRTO formulation only contains linear dynamic models for the DRTO formulation and lower-level MPCs. This chapter introduces various degrees of nonlinearity within the DRTO formulation and control implementation. Firstly, the dynamic plant model which generates the surrogate plant responses is changed to a nonlinear model so that the DRTO formulation can capture the plant behavior in the upper level with better accuracy. Then, the lower-level MPCs and the inner optimization subproblems are also formulated in a nonlinear fashion to introduce nonlinearity to the control problems. The improvement of performances is demonstrated using nonlinear case studies.

The formulations and results in this chapter have been submitted to:

Li, H. and Swartz, C. L. (2017). "Coordination of distributed MPC systems using a nonlinear dynamic plant model with closed-loop prediction", Proceedings of the 13th International Symposium on Process Systems Engineering, in press.

Li, H. and Swartz, C. L. (2017). "Economic Coordination of Distributed Nonlinear MPC Systems using Closed-loop Prediction of a Nonlinear Dynamic Plant", Submitted 
to 6th IFAC Conference on Nonlinear Model Predictive Control, Madison, WN, USA.

\subsection{Problem Formulation for the Inclusion of Non- linear Dynamic Plant Model}

The general architecture of the two-layer coordination scheme for distributed MPCs is shown in Figure 5.1. A nonlinear dynamic plant model is embedded in the upper layer as a surrogate model to generate the plant response. Along the DRTO prediction horizon, a series of MPC optimization subproblems are embedded and generate the control actions applied to the nonlinear dynamic model at each time instance. This sequential closed-loop prediction is thus optimized under a typically economic objective function, and generates set-point trajectories for lower level distributed MPCs to perform subsequent control tracking.

\subsubsection{Model Discretization}

Consider the nonlinear dynamic behaviour of the process plant to be accurately captured by a set of ODEs in the following form:

$$
\begin{aligned}
& \dot{\mathbf{x}}^{\text {DRTO }}(t)=\mathbf{f}^{\text {DRTO }}\left(\mathbf{x}^{\text {DRTO }}(t), \mathbf{u}^{\text {DRTO }}(t)\right) \\
& \mathbf{y}^{\text {DRTO }}(t)=C \mathbf{x}^{\text {DRTO }}(t) \\
& \mathbf{x}^{\text {DRTO }}(0)=\mathbf{x}_{0}, \quad \text { for } t \in\left[0, t_{f}\right]
\end{aligned}
$$

where $\mathbf{x}^{\mathrm{DRTO}}(t) \in R^{n_{x}}$ denotes the differential state vector, $\mathbf{x}^{\mathrm{DRTO}}(0) \in R^{n_{x}}$ the initial state vector, and $\mathbf{u}^{\mathrm{DRTO}}(t) \in R^{n_{u}}$ the input vector. $t_{f}$ represents the duration of the DRTO prediction horizon. To solve the ODE system in the DRTO formulation, the system is discretized using an implicit Euler approximation. The controller sample 


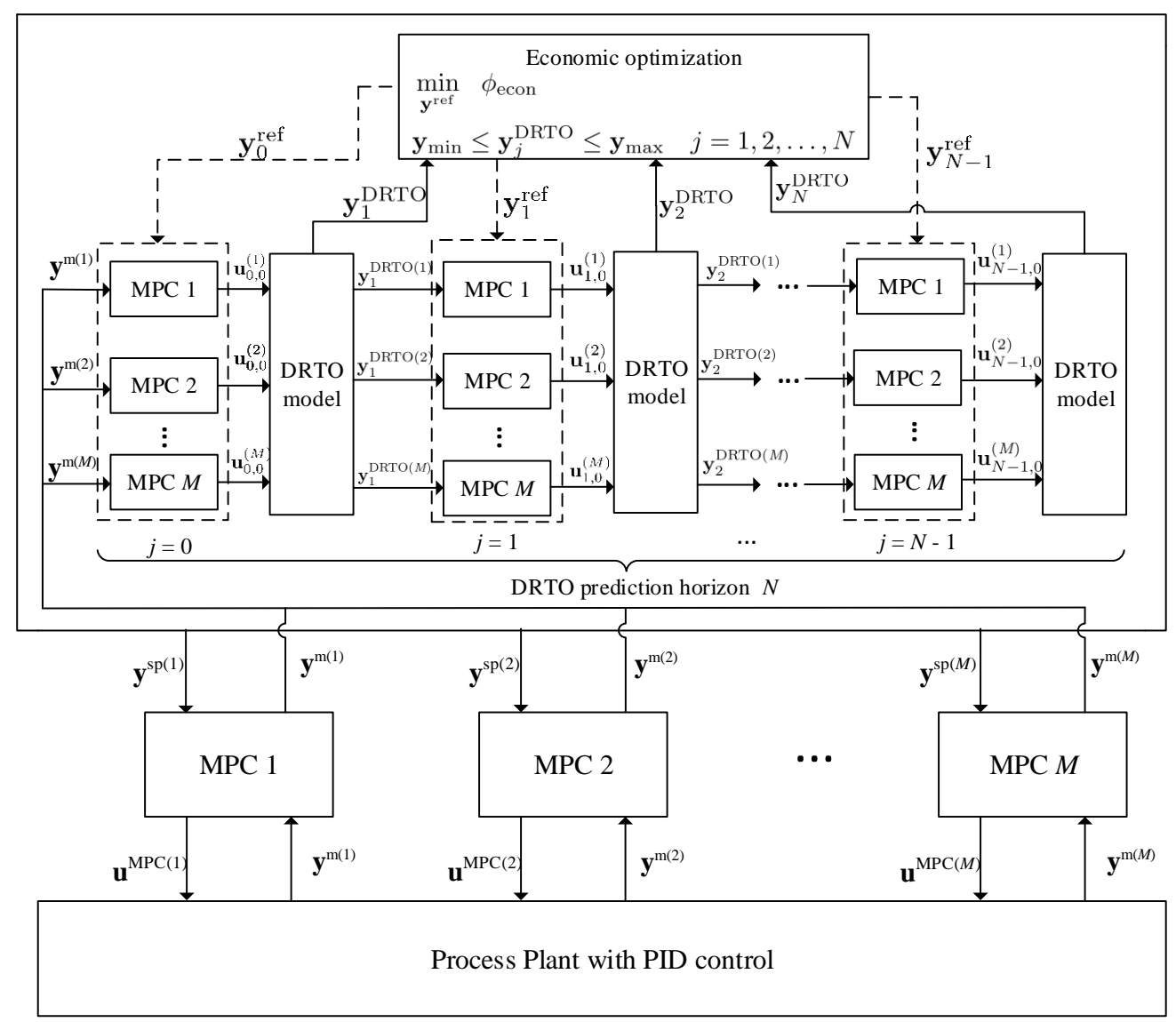

Figure 5.1: The control architecture for coordination of distributed MPCs with CL-DRTO approach. 
time is assumed to be consistent with the finite element interval $\Delta t_{j}$ and the piecewise constant inputs are applied to each finite element along the DRTO prediction horizon. There are various other discretization techniques available to achieve the same effect, such as orthogonal collocation on finite elements. However, implicit Euler integration is demonstrated to be sufficiently accurate to reflect the dynamic behavior generated by the actual plant for the case study considered. The plant is partitioned into subsystems $i \in O$, each of which is controlled by a local MPC based on a linear (or linearized) model of the form $\mathbf{x}_{k+1}^{(i)}=A_{i i} \mathbf{x}_{k}^{(i)}+B_{i i} \mathbf{u}_{k}^{(i)}, \mathbf{y}_{k}^{(i)}=C_{i i} \mathbf{x}_{k}^{(i)}$.

\subsubsection{State and Disturbance Estimation for Nonlinear Plant Model}

Prior to each DRTO execution, the current state estimate $\hat{\mathbf{x}}_{\ell}$ is first calculated based on the discretized model with previous state estimate $\hat{\mathbf{x}}_{\ell-1}$ and the most recently implemented control action $\mathbf{u}_{\ell-1}^{\mathrm{MPC}}$. The predicted state estimate $\hat{\mathbf{x}}_{\ell}$ serves as the initial condition $\mathbf{x}_{0}$ for the DRTO model in the upper level. The disturbance added to the DRTO model is calculated by taking the difference between the current measured outputs and predicted outputs and assumed constant over the horizon:

$$
\begin{aligned}
\frac{\hat{\mathbf{x}}_{\ell}-\hat{\mathbf{x}}_{\ell-1}}{\Delta t_{\ell}} & =\mathbf{f}^{\mathrm{DRTO}}\left(\hat{\mathbf{x}}_{\ell}, \mathbf{u}_{\ell-1}^{\mathrm{MPC}}\right) \\
\mathbf{d}_{\ell}^{\mathrm{DRTO}} & =\mathbf{y}^{\mathrm{m}}-C \hat{\mathbf{x}}_{\ell} \\
\mathbf{x}_{0} & =\hat{\mathbf{x}}_{\ell}
\end{aligned}
$$




\subsubsection{Optimization Formulation}

The dynamic models for the primary optimization problem and MPC optimization subproblems are embedded in the DRTO formulation as Eq. (5.5):

$$
\begin{aligned}
& \min _{\mathbf{y}^{\text {ref }}, \mathbf{u}^{\text {ref }}} \phi^{\text {econ }}\left(\mathbf{x}^{\text {DRTO }}, \mathbf{y}^{\text {DRTO }}, \mathbf{u}^{\text {DRTO }}\right) \\
& \text { s.t. } \frac{\mathbf{x}_{j}^{\mathrm{DRTO}}-\mathbf{x}_{j-1}^{\mathrm{DRTO}}}{\Delta t_{j}}=\mathbf{f}^{\mathrm{DRTO}}\left(\mathbf{x}_{j}^{\mathrm{DRTO}}, \mathbf{u}_{j-1}^{\mathrm{DRTO}}\right), \quad j=1, \ldots, N \\
& \mathbf{y}_{j}^{\mathrm{DRTO}}=C \mathbf{x}_{j}^{\mathrm{DRTO}}+\mathbf{d}_{j}^{\mathrm{DRTO}}, \quad j=1, \ldots, N \\
& \mathbf{g}^{\mathrm{DRTO}}\left(\mathbf{x}^{\mathrm{DRTO}}, \mathbf{y}^{\mathrm{DRTO}}\right) \geq \mathbf{0} \\
& \mathbf{h}_{i}^{\mathrm{ref}}\left(\mathbf{y}^{\mathrm{ref}}, \mathbf{u}^{\mathrm{ref}}, \mathbf{y}^{\mathrm{sp}(i)}, \mathbf{u}^{\mathrm{sp}(i)}\right)=\mathbf{0}, \quad i \in O \\
& \mathbf{g}^{\text {ref }}\left(\mathbf{y}^{\text {ref }}, \mathbf{u}^{\text {ref }}\right) \geq \mathbf{0} \\
& \mathbf{u}_{j}^{\mathrm{DRTO}(i)}=\mathbf{u}_{j, 0}^{(i)}, \quad j=0, \ldots, N-1 \\
& \mathbf{u}_{j, 0}^{(i)} \in \arg \min _{\mathbf{u}_{j, k}^{(i)}} \phi_{j}^{\mathrm{MPC}(\mathrm{i})}\left(\mathbf{y}_{j, k}^{(i)}, \mathbf{y}_{j, k}^{\mathrm{sp}(i)}, \mathbf{u}_{j, k}^{(i)}, \mathbf{u}_{j, k}^{\mathrm{sp}(i)}\right) \\
& \text { s.t. } \quad \mathbf{x}_{j, k+1}^{(i)}=A_{i i} \mathbf{x}_{j, k}^{(i)}+B_{i i} \mathbf{u}_{j, k}^{(i)}, \quad k=0, \ldots, m-1 \\
& \mathbf{x}_{j, k+1}^{(i)}=A_{i i} \mathbf{x}_{j, k}^{(i)}+B_{i i} \mathbf{u}_{j, m-1}^{(i)}, \quad k=m, \ldots, p-1 \\
& \mathbf{y}_{j, k}^{(i)}=C_{i i} \mathbf{x}_{j, k}^{(i)}+\mathbf{d}_{j, k}^{(i)}, \quad k=1, \ldots, p \\
& \mathbf{d}_{0, k}^{(i)}=\mathbf{y}^{\mathrm{m}(i)}-C_{i i} \mathbf{x}_{0}^{(i)}, \quad k=1, \ldots, p \\
& \mathbf{d}_{j, k}^{(i)}=\mathbf{y}_{j}^{\mathrm{DRTO}(i)}-C_{i i} \mathbf{x}_{j-1,1}^{(i)}, \quad k=1, \ldots, p, j>0 \\
& \mathbf{u}_{\min }^{(i)} \leq \mathbf{u}_{j, k}^{(i)} \leq \mathbf{u}_{\max }^{(i)}, \quad k=0, \ldots, m-1
\end{aligned}
$$

The closed-loop DRTO formulation consists of a primary optimization problem based on the nonlinear dynamic system to predict closed-loop response, and inner MPC optimization subproblems based on linear models of the plant subsystems to compute control actions. $\phi^{\text {econ }}$ denotes the objective function that captures the performance criterion to be optimized. The main decision variables for the primary optimization problem are reference trajectories $\mathbf{y}^{\text {ref }}$ and $\mathbf{u}^{\text {ref }}$, from which the set-point trajectories 
for distributed MPCs $\mathbf{y}_{j, k}^{\mathrm{sp}(\mathrm{i})}$ and $\mathbf{u}_{j, k}^{\mathrm{sp}(\mathrm{i})}$ are extracted, while the decision variables in the inner MPC optimization subproblems are the control actions $\mathbf{u}_{j, k}^{(i)}$. $\mathbf{h}^{\text {ref }}$ represents the mapping of the DRTO reference trajectories to the MPC set-point trajectories, and can be used to enforce the trajectories to be constant within each DRTO interval over the DRTO prediction horizon, which can be explicitly represented as:

$$
\begin{aligned}
& \mathbf{y}_{j, k}^{\mathrm{sp}(\mathrm{i})}=E_{y}^{(i)} \mathbf{y}_{j+k}^{\mathrm{ref}}, \quad k=1, \ldots, p \\
& \mathbf{u}_{j, k}^{\mathrm{sp}(\mathrm{i})}=E_{u}^{(i)} \mathbf{u}_{j+k}^{\mathrm{ref}}, \quad k=0, \ldots, m-1
\end{aligned}
$$

where $E_{y}^{(i)} \in R^{n_{y}^{(i)} \times n_{y}}$ and $E_{u}^{(i)} \in R^{n_{u}^{(i)} \times n_{u}}$ are matrices that map the full plant outputs and inputs to the subsystems. $\mathrm{g}^{\text {ref }}$ represents the upper and lower bounds of the trajectories. $\mathbf{x}^{\text {DRTO }}, \mathbf{y}^{\text {DRTO }}$, and $\mathbf{u}^{\text {DRTO }}$ are composite vectors of $\mathbf{x}_{j}^{\text {DRTO }}, \mathbf{y}_{j}^{\text {DRTO }}$, and $\mathbf{u}_{j}^{\text {DRTO }}$ respectively over the DRTO horizon. $\mathbf{u}_{j}^{\text {DRTO }(i)}$ and $\mathbf{y}_{j}^{\text {DRTO }(i)}$ are subvectors of $\mathbf{u}_{j}^{\text {DRTO }}$ and $\mathbf{y}_{j}^{\text {DRTO }}$ corresponding to the inputs and outputs of MPC subsystem $i$. A quadratic MPC objective function is used to yield a QDMC formulation as proposed in Garcia and Morshedi 1986 (see also Maciejowski 2002). The disturbance estimate $\mathbf{d}_{j, k}^{(i)}$ is determined as proposed in Cutler and Ramaker 1979 and Garcia and Morshedi [1986], and utilizes the plant measurement $\mathbf{y}^{\mathrm{m}(i)}$ for the first set of MPC subproblems, and the DRTO model output, $\mathbf{y}_{j}^{\mathrm{DRTO}(i)}$, for subsequent time steps in the DRTO horizon, where $\mathbf{y}^{\mathrm{m}(i)}$ is a subvector of the plant measurements corresponding to MPC subsystem $i$.

The multi-level optimization formulation of the closed-loop DRTO problem can be transformed into a single-level optimization problem by reformulating the MPC quadratic programming subproblems as a set of constraints using the Karush-Kuhn-Tucker (KKT) optimality conditions, as proposed by Baker and Swartz 2008]. This cor- 
respondence for a general convex quadratic programming problem is:

$$
\begin{array}{ccc} 
& \mathrm{QP} & \text { KKT conditions } \\
\min _{\mathbf{z}} & \frac{1}{2} \mathbf{z}^{T} H \mathbf{z}+g^{T} \mathbf{z} & H \mathbf{z}+\mathbf{g}-A^{T} \boldsymbol{\nu}-\boldsymbol{\eta}=\mathbf{0} \\
\text { s.t. } & A \mathbf{z}=\mathbf{b}, \quad \mathbf{z} \geq \mathbf{0} & A \mathbf{z}=\mathbf{b}, \quad z_{i} \eta_{i}=0, \quad(\mathbf{z}, \boldsymbol{\eta}) \geq \mathbf{0}
\end{array}
$$

where variable $\mathbf{z}$ denotes a generalized variable within the QP problem. The presence of the complementarity constraints in the reformulated problem may be effectively handled through an exact penalty formulation in which they are included as a penalty term in the objective function Ralph and Wright, 2004; Jamaludin and Swartz, 2017a. The DRTO formulation are solved using AMPL with the IPOPT solver, and the lower-level control calculations and continuous plant simulation are implemented in MATLAB using the ode15s integrator.

\subsubsection{Case Study}

We consider a reactor-separator network studied by Baldea and Daoutidis [2007]. A zero order reaction converts component $\mathrm{A}$ to component $\mathrm{B}$ in the reactor, and the product is then fed to the separator. The mole transfer rate between the vapor and liquid for component $i \in\{\mathrm{A}, \mathrm{B}, \mathrm{I}\}$ in the vessel is modeled with the rate expression, $N_{j}=K_{j} \alpha\left(y_{j}-\left(P_{j}^{s} / P c\right) x_{j}\right)\left(M_{L} / \rho_{L}\right)$. The differential equations used for this system are given below, where $M_{R}, M_{V}, M_{L}$ denote the molar holdup in the reactor, condenser vapor and liquid phase, and $N=N_{A}+N_{B}+N_{I}$. For simplicity, the molar hold-ups 
are assumed to be perfectly controlled.

$$
\begin{aligned}
& \dot{y}_{A, R}=\frac{1}{M_{R}}\left[F_{o}\left(y_{A, o}-y_{A, R}\right)+R\left(y_{A}-y_{A, R}\right)-k_{1} M_{R} y_{A, R}\right] \\
& \dot{y}_{I}=\frac{1}{M_{V}}\left[F\left(y_{I, R}-y_{I}\right)-N_{I}+y_{I} N\right] \\
& \dot{y}_{I, R}=\frac{1}{M_{R}}\left[F_{o}\left(y_{I, o}-y_{I, R}\right)+R\left(y_{I}-y_{I, R}\right)\right] \\
& \dot{x}_{A}=\frac{1}{M_{L}}\left[N_{A}+x_{A} N\right] \\
& \dot{y}_{A}=\frac{1}{M_{V}}\left[F\left(y_{A, R}-y_{A}\right)-N_{A}+y_{A} N\right] \\
& \dot{x}_{I}=\frac{1}{M_{L}}\left[N_{I}+x_{I} N\right]
\end{aligned}
$$

The nominal values for all process parameters are summarized in Baldea and Daoutidis 2007]. The manipulated and control variables selected for the reactor are raw feed $F_{o}$ and vapor composition of component A, $y_{A, R}$, respectively. The manipulated and controlled variables selected for the separator are condenser pressure $P_{c}$ and liquid composition of component $\mathrm{A}, x_{A}$, respectively. The tuning parameters for distributed MPCs and the DRTO formulation are summarized in Table 5.3, It is noted that the first move suppression weight value is small due to the small nominal value of the condenser pressure (0.338 MPa compared to feed flow rate $100 \mathrm{~mol} / \mathrm{min})$. In this case study, the system is given pre-determined targets for two outputs that are required to be tracked; the DRTO objective function is consequently formulated in a least-squares fashion. However, other forms of linear or nonlinear objective function can be readily used. The system is regulated separately for both centralized and decentralized MPC. For each case, the associated DRTO formulation is added to generate set-point trajectories for those MPCs. The performance is quantified using the sum-squared-errors between the plant dynamics and final target values. Figure 5.2 shows the set-point trajectories and response process dynamics for distributed cases using linear and nonlinear DRTO formulations. While the difference between the nonlinear and linear DRTO implementations appears slight, numerical results confirm the superior performance of the nonlinear approach. Table 5.2 summarizes 
the performance for several configurations: centralized and distributed DRTO with a nonlinear DRTO plant model, centralized and distributed DRTO with a linear DRTO plant model, and decentralized MPC with constant set-point trajectories corresponding to the set-point targets. From the results it can be seen that the nonlinear DRTO formulation outperforms the linear counterpart, which aligns with expectation since the DRTO formulation can generate a more accurate plant response based on the nonlinear model. The table also shows that the distributed cases experience only a slight performance drop when compared to the centralized counterparts due to the ability of DRTO formulation to predict the plant behavior based on a complete plant model that is not available to distributed MPCs. The performance drop can be justified by the additional flexibility and reliability offered by the distributed architecture over the centrazlied counterpart.

Table 5.1: DRTO and MPC parameters for the process

\begin{tabular}{lll}
\hline Parameter & Description & Value (MPC1 \& MPC2) \\
\hline$\Delta t_{D R T O}$ & DRTO sample time & 20 \\
$\Delta t_{M P C}$ & MPC sample time & 10 \\
$\mathrm{~N}$ & Optimization horizon & 10 \\
$\mathrm{P}$ & Prediction Horizon & 5 \\
$\mathrm{M}$ & Control Horizon & 3 \\
$\mathrm{Q}$ & Output tracking weight & $10 \& 2$ \\
$\mathrm{R}$ & Move suppression weight & $10^{-4} \& 5$ \\
$y_{\min }, y_{\max }$ & Output and setpoint bounds & {$\left[\begin{array}{ll}0 & 1\end{array}\right] \&\left[\begin{array}{ll}0 & 1\end{array}\right]$} \\
$u_{\min }, u_{\max }$ & Input bounds & {$\left[\begin{array}{ll}0 & 250\end{array}\right] \&\left[\begin{array}{ll}0 & 1\end{array}\right]$} \\
\hline
\end{tabular}



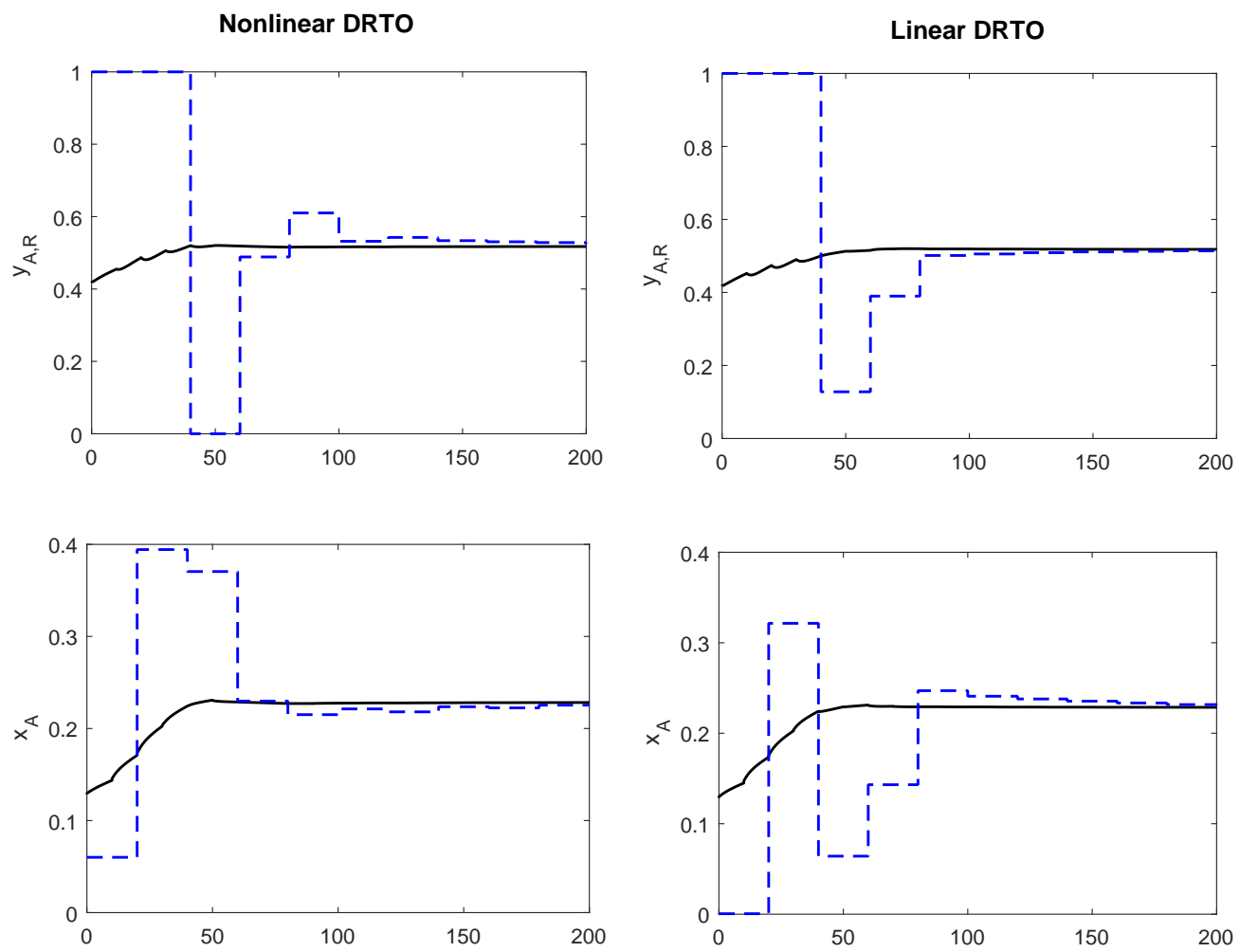

Figure 5.2: Set-points and output response for distributed MPC cases

\subsection{Problem Formulation for Coordination of Non- linear MPCs}

The coordination scheme for distributed NMPCs using the two-layer approach is illustrated in Fig. 5.3. In order to generate a predicted plant response, a nonlinear dynamic plant model is embedded in the upper layer. The control actions applied to the nonlinear dynamic model are produced by a series of nonlinear MPC optimization subproblems embedded along the DRTO prediction horizon. The entire optimization problem can be optimized under either a least-squares objective for tracking targets or an economic objective function to maximize profit. The set-point trajectories are 
Table 5.2: The summary of performance under different configurations

\begin{tabular}{llll}
\hline Configuration & Control architecture & $\sum S S E$ & $\begin{array}{l}\text { performance } \\
\text { loss }\end{array}$ \\
\hline centralized & nonlinear DRTO + MPC & 2.473 & $0 \%$ \\
distributed & nonlinear DRTO + MPC & 2.556 & $3.36 \%$ \\
centralized & linear DRTO + MPC & 2.743 & $10.91 \%$ \\
distributed & linear DRTO + MPC & 2.790 & $12.82 \%$ \\
decentralized & MPC & 6.61 & $167.29 \%$ \\
\hline
\end{tabular}

\section{$\min \varphi_{\text {econ }}$}

s.t. nonlinear plant and local NMPC models constraints on reference trajectories constraints on predicted closed-loop response

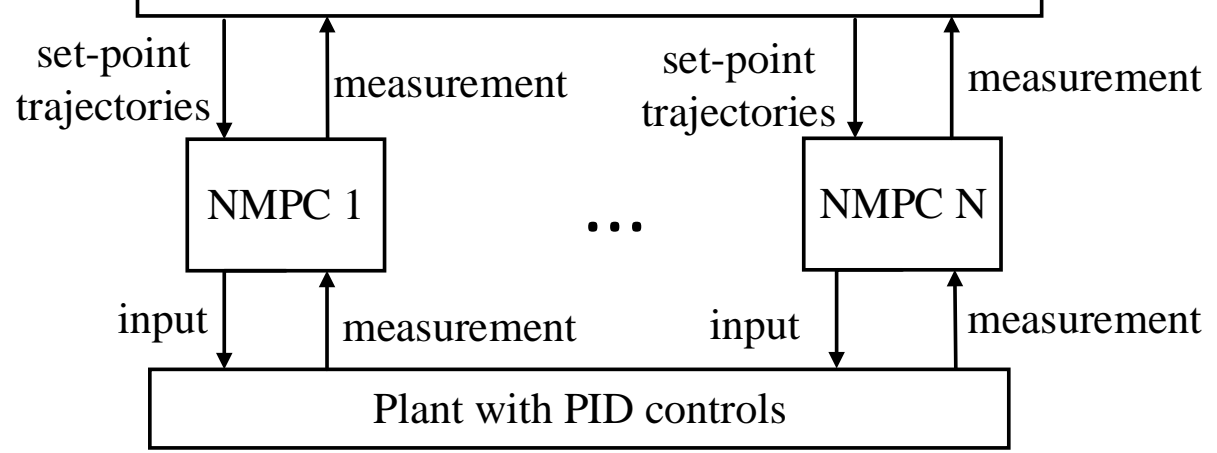

Figure 5.3: The control architecture for coordination of distributed MPCs with closed-loop DRTO approach.

the decision variables of this optimization problem and assigned to the lower level distributed NMPC systems to perform subsequent control tracking. 


\subsubsection{Model Discretization}

Consider the nonlinear dynamic behavior of the process plant to be accurately captured by a set of ordinary differential equations (ODEs) in the following form:

$$
\begin{aligned}
& \dot{\mathbf{x}}^{\mathrm{DRTO}}(t)=\mathbf{f}^{\mathrm{DRTO}}\left(\mathbf{x}^{\mathrm{DRTO}}(t), \mathbf{u}^{\mathrm{DRTO}}(t)\right) \\
& \mathbf{y}^{\mathrm{DRTO}}(t)=C \mathbf{x}^{\mathrm{DRTO}}(t) \\
& \mathbf{x}^{\mathrm{DRTO}}(0)=\mathbf{x}_{0}, \quad \text { for } t \in\left[0, t_{f}\right]
\end{aligned}
$$

where $\mathbf{x}^{\mathrm{DRTO}}(t) \in R^{n_{x}}$ denotes the differential state vector, $\mathbf{x}^{\mathrm{DRTO}}(0) \in R^{n_{x}}$ the initial state vector, and $\mathbf{u}^{\mathrm{DRTO}}(t) \in R^{n_{u}}$ the input vector. $t_{f}$ represents the duration of the DRTO prediction horizon. $\mathbf{x}_{0}$ represents the initial conditions for the full plant states.

The plant is partitioned into subsystems $i=1, \ldots, M$, each of which is controlled by a local nonlinear MPC based on the form:

$$
\begin{aligned}
\dot{\mathbf{x}}^{(i)}(t) & =\mathbf{f}^{(i)}\left(\mathbf{x}^{(i)}(t), \mathbf{u}^{(i)}(t)\right) \\
\mathbf{y}^{(i)}(t) & =C^{(i)} \mathbf{x}^{(i)}(t) \\
\mathbf{x}^{(i)}(0) & =\mathbf{x}_{0}^{(i)}, \quad \text { for } t \in\left[0, t_{p}\right]
\end{aligned}
$$

where $\mathbf{x}^{(i)}(t) \in R^{n_{x}^{(i)}}$ denotes the differential state vector, $\mathbf{x}^{(i)}(0) \in R^{n_{x}^{(i)}}$ the initial state vector, and $\mathbf{u}^{(i)}(t) \in R^{n_{u}^{(i)}}$ the input vector. $t_{p}$ represents the duration of the NMPC prediction horizon. In this work, we consider open-loop stable systems and a sufficiently long prediction horizon to maintain closed-loop stability for the transitions considered. Alternative NMPC formulations that guarantee closed-loop stability under certain conditions through inclusion of a terminal penalty term and/or constraint can be accommodated with corresponding adjustments to the DRTO formulation.

To solve the ODE systems in the DRTO formulation, the systems are discretized using 
an implicit Euler approximation. Piecewise constant control inputs are assumed over the controller sample time $\Delta t_{j}$, which for the present application is also chosen as the integration step size. Various other discretization techniques may be used, such as orthogonal collocation on finite elements and multiple shooting Allgower et al., 2004. However, implicit Euler integration is demonstrated to be sufficiently accurate to reflect the dynamic behavior generated by the actual plant for the case study considered.

\subsubsection{State and Disturbance Estimation for Nonlinear Plant Model}

Prior to each DRTO execution, the current state estimate $\hat{\mathbf{x}}_{\ell}$ for the nonlinear dynamic plant and $\hat{\mathbf{x}}_{\ell}^{(i)}$ for the $i$ th NMPC are first calculated based on their associated models applied to the previous state estimate $\hat{\mathbf{x}}_{\ell-1}$ and $\hat{\mathbf{x}}_{\ell-1}^{(i)}$, respectively, utilizing the most recently implemented control action $\mathbf{u}_{\ell-1}^{\mathrm{MPC}}$. The predicted state estimate $\hat{\mathbf{x}}_{\ell}$ serves as the initial condition $\mathbf{x}_{0}$ for the DRTO model, and $\hat{\mathbf{x}}_{\ell}^{(i)}$ as the initial condition $\mathbf{x}_{0}^{(i)}$ for the NMPC model in the upper level. The disturbance added to the DRTO model is calculated by taking the difference between the current measured outputs and predicted outputs and assumed constant over the horizon. The relevant expressions 
are as follows:

$$
\begin{aligned}
\frac{\hat{\mathbf{x}}_{\ell}-\hat{\mathbf{x}}_{\ell-1}}{\Delta t_{\ell}} & =\mathbf{f}^{\mathrm{DRTO}}\left(\hat{\mathbf{x}}_{\ell}, \mathbf{u}_{\ell-1}^{\mathrm{MPC}}\right) \\
\mathbf{d}_{\ell}^{\mathrm{DRTO}} & =\mathbf{y}^{\mathrm{m}}-C \hat{\mathbf{x}}_{\ell} \\
\mathbf{x}_{0} & =\hat{\mathbf{x}}_{\ell} \\
\frac{\hat{\mathbf{x}}_{\ell}^{(i)}-\hat{\mathbf{x}}_{\ell-1}^{(i)}}{\Delta t_{\ell}} & =\mathbf{f}^{(i)}\left(\hat{\mathbf{x}}_{\ell}^{(i)}, \mathbf{u}_{\ell-1}^{\mathrm{MPC}}\right) \\
\mathbf{d}_{\ell}^{(i)} & =\mathbf{y}^{\mathrm{m}(i)}-C \hat{\mathbf{x}}_{\ell}^{(i)} \\
\mathbf{x}_{0}^{(i)} & =\hat{\mathbf{x}}_{\ell}^{(i)}
\end{aligned}
$$

where $\mathbf{y}^{\mathrm{m}}$ is the full set of plant measurements, and $\mathbf{y}^{\mathrm{m}(i)}$ is the subvector of measurements corresponding to subsystem. 


$$
\begin{aligned}
& \min _{\mathbf{y}^{\mathrm{ref}}, \mathbf{u}^{\mathrm{ref}}} \phi^{\mathrm{DRTO}}\left(\mathbf{x}^{\mathrm{DRTO}}, \mathbf{y}^{\mathrm{DRTO}}, \mathbf{u}^{\mathrm{DRTO}}\right) \\
& \text { s.t. } \quad \mathbf{f}^{\mathrm{DRTO}}\left(\mathbf{x}_{j}^{\mathrm{DRTO}}, \mathbf{x}_{j-1}^{\mathrm{DRTO}}, \mathbf{u}_{j-1}^{\mathrm{DRTO}}\right)=\mathbf{0}, \quad j=1, \ldots, N \\
& \mathbf{y}_{j}^{\text {DRTO }}=C \mathbf{x}_{j}^{\text {DRTO }}+\mathbf{d}_{j}^{\text {DRTO }}, j=1, \ldots, N \\
& \mathbf{g}^{\text {DRTO }}\left(\mathbf{x}^{\text {DRTO }}, \mathbf{y}^{\text {DRTO }}\right) \geq \mathbf{0} \\
& \mathbf{h}_{i}^{\mathrm{ref}}\left(\mathbf{y}^{\mathrm{ref}}, \mathbf{u}^{\mathrm{ref}}, \mathbf{y}^{\mathrm{sp}(i)}, \mathbf{u}^{\mathrm{sp}(i)}\right)=\mathbf{0}, \quad i=1, \ldots, M \\
& \mathbf{g}^{\mathrm{ref}}\left(\mathbf{y}^{\mathrm{ref}}, \mathbf{u}^{\mathrm{ref}}\right) \geq \mathbf{0} \\
& \mathbf{u}_{j}^{\operatorname{DRTO}(i)}=\mathbf{u}_{j, 0}^{(i)}, \quad j=0, \ldots, N-1 \\
& \mathbf{u}_{j, 0}^{(i)} \in \arg \min _{\mathbf{u}_{j, k}^{(i)}} \phi_{j}^{\mathrm{MPC}(i)}\left(\mathbf{y}_{j, k}^{(i)}, \mathbf{y}_{j, k}^{\mathrm{sp}(i)}, \mathbf{u}_{j, k}^{(i)}, \mathbf{u}_{j, k}^{\mathrm{sp}(i)}\right) \\
& \text { s.t. } \quad \mathbf{f}^{(i)}\left(\mathbf{x}_{j, k+1}^{(i)}, \mathbf{x}_{j, k}^{(i)}, \mathbf{u}_{j, k}^{(i)}\right)=\mathbf{0}, \quad k=0, \ldots, m-1 \\
& \mathbf{f}^{(i)}\left(\mathbf{x}_{j, k+1}^{(i)}, \mathbf{x}_{j, k}^{(i)}, \mathbf{u}_{j, m-1}^{(i)}\right)=\mathbf{0}, \quad k=m, \ldots, p-1 \\
& \mathbf{y}_{j, k}^{(i)}=C_{i} \mathbf{x}_{j, k}^{(i)}+\mathbf{d}_{j, k}^{(i)}, \quad k=1, \ldots, p \\
& \mathbf{d}_{0, k}^{(i)}=\mathbf{y}^{\mathrm{m}(i)}-C_{i} \mathbf{x}_{0}^{(i)}, \quad k=1, \ldots, p \\
& \mathbf{d}_{j, k}^{(i)}=\mathbf{y}_{j}^{\mathrm{DRTO}(i)}-C_{i} \mathbf{x}_{j-1,1}^{(i)}, \quad k=1, \ldots, p, j>0 \\
& \mathbf{u}_{\min }^{(i)} \leq \mathbf{u}_{j, k}^{(i)} \leq \mathbf{u}_{\max }^{(i)}, \quad k=0, \ldots, m-1
\end{aligned}
$$

\subsubsection{Optimization Formulation}

The closed-loop DRTO formulation comprises a primary optimization problem based on the full dynamic model to predict the closed-loop response of the actual plant, and inner MPC optimization subproblems based on plant subsystem models to compute control actions to be implemented on the plant to generate surrogate outputs.

The dynamic models for both parts are nonlinear. The formulation is shown in (5.20). The objective function $\phi^{\mathrm{DRTO}}$ specifies the performance criterion to be optimized. The reference trajectories $\mathbf{y}^{\text {ref }}$ and $\mathbf{u}^{\text {ref }}$ are constructed as decision variables, from which 


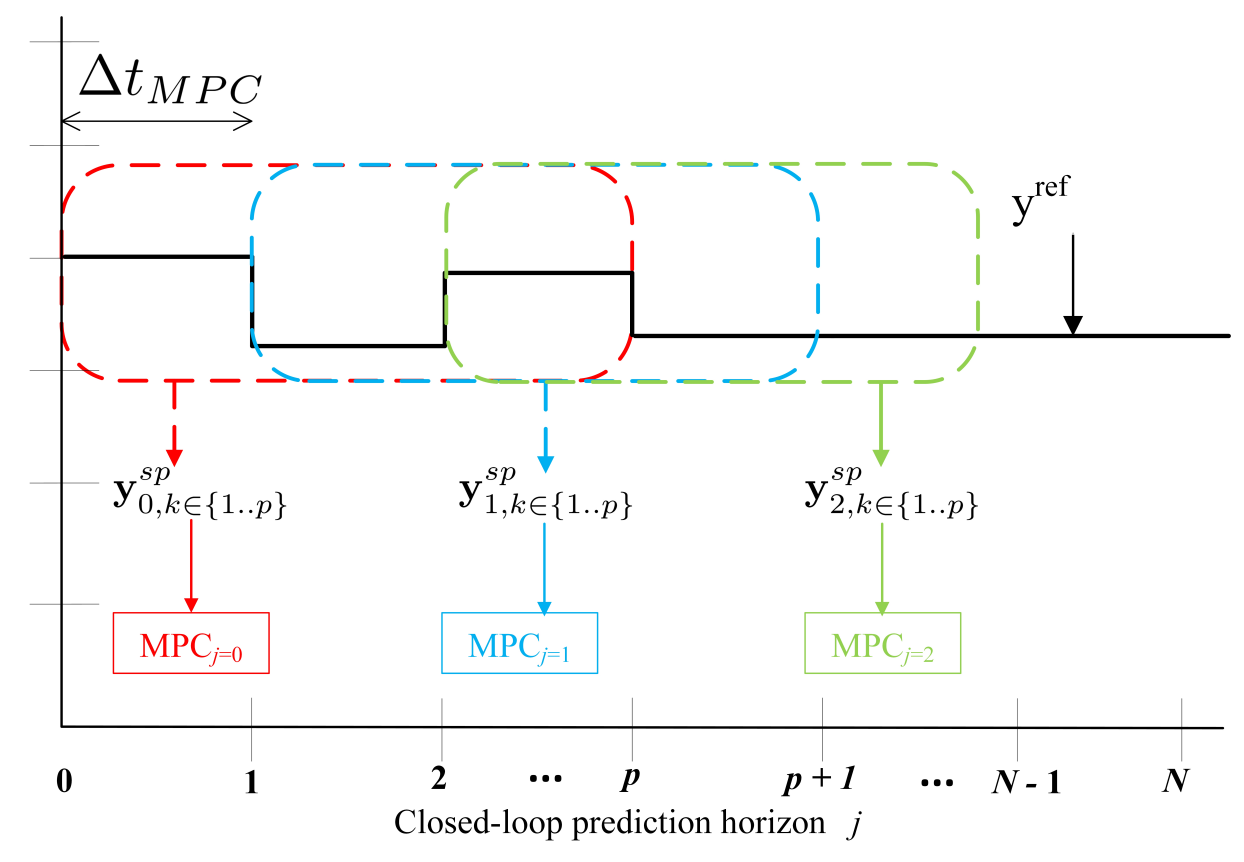

Figure 5.4: The set-point trajectory extractions from the reference trajectory.

the set-point trajectories for distributed MPCs $\mathbf{y}_{j, k}^{\mathrm{sp}(i)}$ and $\mathbf{u}_{j, k}^{\mathrm{sp}(i)}$ are extracted and assigned to the lower-level NMPCs for tracking. The extraction of the set-point trajectories for the embedded NMPCs along the DRTO prediction horizon is illustrated in Fig. 5.4, with the subsystem indices omitted.

The equality constraints $\mathbf{h}_{i}^{\text {ref }}$ represent such extraction of MPC set-point trajectories, with detailed expressions given by:

$$
\begin{aligned}
& \mathbf{y}_{j, k}^{\mathrm{sp}(i)}=E_{y}^{(i)} \mathbf{y}_{j+k}^{\mathrm{ref}}, \quad k=1, \ldots, p \\
& \mathbf{u}_{j, k}^{\mathrm{sp}(i)}=E_{u}^{(i)} \mathbf{u}_{j+k}^{\mathrm{ref}}, \quad k=0, \ldots, m-1
\end{aligned}
$$

where $E_{y}^{(i)} \in R^{n_{y}^{(i)} \times n_{y}}$ and $E_{u}^{(i)} \in R^{n_{u}^{(i)} \times n_{u}}$ are matrices that map the full plant outputs and inputs to the subsystem outputs and inputs. For instance, if a system with three outputs is partitioned into two subsystems with the first subsystem containing the first output, and the second subsystem containing the second and third outputs, the 
$E_{y}^{(i)}$ matrices can be written as:

$$
E_{y}^{(1)}=\left[\begin{array}{lll}
1 & 0 & 0
\end{array}\right], \quad E_{y}^{(2)}=\left[\begin{array}{lll}
0 & 1 & 0 \\
0 & 0 & 1
\end{array}\right]
$$

For the inner optimization subproblems, the decision variables are the control actions $\mathbf{u}_{j, k}^{(i)}$, which are in turn equated to the inputs applied to the plant by direct mapping. The bounds for generated trajectories can be specified in $\mathbf{g}^{\text {ref }} \cdot \mathbf{x}^{\text {DRTO }}, \mathbf{y}^{\text {DRTO }}$, and $\mathbf{u}^{\text {DRTO }}$ are composite vectors of $\mathbf{x}_{j}^{\text {DRTO }}, \mathbf{y}_{j}^{\text {DRTO }}$, and $\mathbf{u}_{j}^{\text {DRTO }}$ respectively over the DRTO horizon. $\mathbf{u}_{j}^{\mathrm{DRTO}(i)}$ and $\mathbf{y}_{j}^{\mathrm{DRTO}(i)}$ are subvectors of $\mathbf{u}_{j}^{\mathrm{DRTO}}$ and $\mathbf{y}_{j}^{\mathrm{DRTO}}$ corresponding to the inputs and outputs of MPC subsystem $i$.

The disturbance estimate $\mathbf{d}_{j, k}^{(i)}$ is determined as proposed in Cutler and Ramaker 1979 and Garcia and Morshedi [1986, and utilizes the plant measurement $\mathbf{y}^{\mathrm{m}(i)}$ for the first set of MPC subproblems, and the DRTO model output, $\mathbf{y}_{j}^{\mathrm{DRTO}(i)}$, for subsequent time steps in the DRTO horizon.

\subsubsection{Solution Strategy}

The multi-level optimization formulation of the closed-loop DRTO problem is transformed into a single-level optimization problem by reformulating the NMPC subproblems as a set of constraints using the Karush-Kuhn-Tucker (KKT) optimality conditions, as proposed by Baker and Swartz 2008 and Jamaludin and Swartz 2017b for linear MPC. Consider the general nonlinear MPC problem below:

$$
\begin{array}{ll}
\min _{\mathbf{z}} & \frac{1}{2} \mathbf{z}^{T} H \mathbf{z}+\mathbf{g}^{T} \mathbf{z} \\
\text { s.t. } & \mathbf{f}(\mathbf{z})=\mathbf{0} \\
& \mathbf{z} \geq \mathbf{0}
\end{array}
$$


where $\mathbf{z}$ is a composite vector of variables in the NMPC formulation. The Lagrangian of this problem can be written as:

$$
\mathcal{L}(\mathbf{z})=\frac{1}{2} \mathbf{z}^{T} H \mathbf{z}+\mathbf{g}^{T} \mathbf{z}-\boldsymbol{\lambda}^{T} \mathbf{f}(\mathbf{z})-\boldsymbol{\eta}^{T} \mathbf{z}
$$

Thus, the KKT conditions can be expressed as:

$$
\begin{aligned}
\nabla_{\mathbf{z}} \mathcal{L} & =H \mathbf{z}+\mathbf{g}-\boldsymbol{\lambda}^{T} \mathbf{f}_{\mathbf{z}}-\boldsymbol{\eta}=\mathbf{0} \\
\mathbf{f}(\mathbf{z}) & =\mathbf{0} \\
z_{i} \eta_{i} & =0, \quad(\mathbf{z}, \boldsymbol{\eta}) \geq \mathbf{0}
\end{aligned}
$$

The presence of the complementarity constraints in the reformulated problem is handled through an exact penalty formulation in which they are included as a penalty term in the objective function Ralph and Wright, 2004, Jamaludin and Swartz, 2017a. The DRTO and NMPC formulations are solved using AMPL with the IPOPT solver at their associated sample time. The continuous plant simulation is carried out in MATLAB using the ode15s integrator.

\subsection{Case Study}

MPC coordination using the DRTO formulation is demonstrated by applying the

formulation to two CSTRs in series, utilizing the model presented in Loeblein and Perkins [1998]. The process is illustrated in Fig. 5.5. All assumptions and process parameter values are as in the original paper. The levels in both CSTRs are also assumed to be perfectly controlled. The model is derived from material and energy 


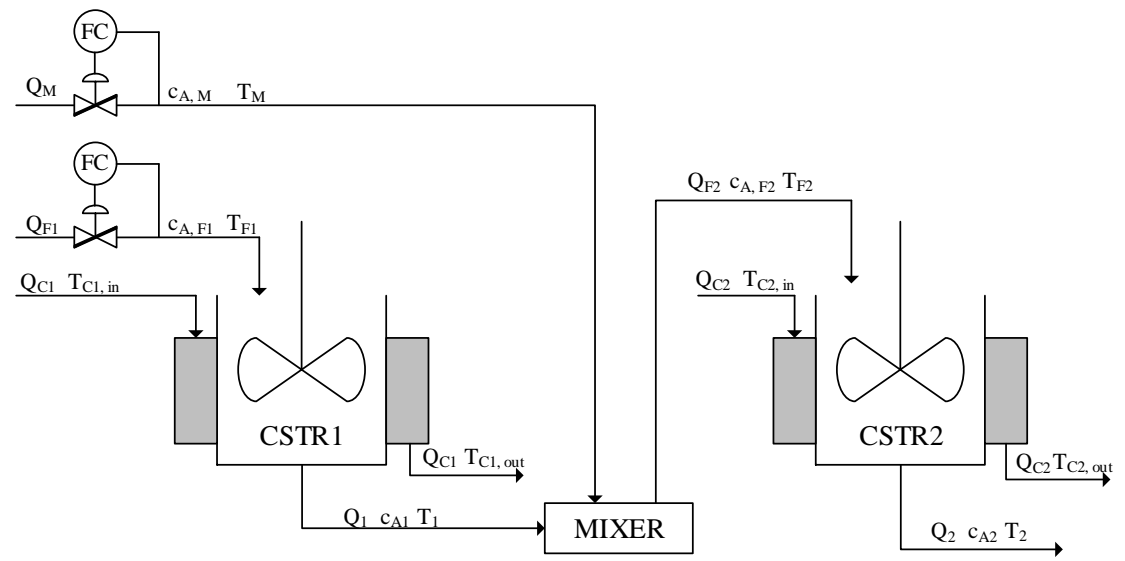

Figure 5.5: The process schematics for the two-CSTRs process

balances, given by

$$
\begin{aligned}
\frac{d c_{A i}}{d t} & =Q_{F i} c_{A, F i}-Q_{i} c_{A i}-c_{A i} V k_{0, I} e^{\left(-\frac{E_{I}}{R T_{i}}\right)} \\
\frac{d c_{B i}}{d t} & =Q_{F i} c_{B, F i}-Q_{i} c_{B i}+c_{A i} V k_{0, I} e^{\left(-\frac{E_{I}}{R T_{i}}\right)} \\
& -c_{B i} V k_{0, I I} e^{-\frac{E_{I I}}{R T_{i}}} \\
\frac{d c_{C i}}{d t}= & Q_{F i} c_{C, F i}-Q_{i} c_{C i}+c_{B i} V k_{0, I I} e^{\left(-\frac{E_{I I}}{R T_{i}}\right)} \\
\frac{d T_{i}}{d t}= & Q_{F i} T_{F i}-Q_{i} T_{i}-\frac{\Delta H_{R, I}}{\rho c_{p}} c_{A i} V k_{0, I} e^{\left(-\frac{E_{I}}{R T_{i}}\right)} \\
& -\frac{\Delta H_{R, I I}}{\rho c_{p}} c_{B i} V k_{0, I I} e^{\left(-\frac{E_{I I I}}{R T_{i}}\right)}-\frac{P_{c i}}{\rho c_{p}} \quad i=1,2
\end{aligned}
$$


with the following algebraic equations:

$$
\begin{aligned}
q_{c o o l, i} & =\frac{P_{c i}}{\rho c_{p}}=\frac{U A}{\rho c_{p}} \Delta T_{l n, i}=U_{a} \Delta T_{l n, i} \\
\Delta T_{l n, i} & =\frac{T_{c i, o u t}-T_{c i, i n}}{\ln \left(T_{i}-T_{c i, i n}\right) /\left(T_{i}-T_{c i, o u t}\right)} \\
q_{c o o l, i} & =Q_{c i}\left(T_{c i, \text { out }}-T_{c i, i n}\right), \quad i=1,2 \\
Q_{F 2} & =Q_{1}+Q_{M} \\
Q_{F 2} c_{i, F 2} & =Q_{1} c_{i, 1}+Q_{M} c_{i, M} \quad i=A, B, C \\
Q_{F 2} T_{F 2} & =Q_{1} T_{1}+Q_{M} T_{M}
\end{aligned}
$$

The following constraints are imposed to meet safety, supply, process and product quality restrictions:

$$
\begin{array}{r}
T_{1} \leq 350 \mathrm{~K}, \quad T_{2} \leq 350 \mathrm{~K} \\
Q_{F 1}+Q_{M} \leq 0.8 \mathrm{~m}^{3} / \mathrm{s} \\
T_{c 1, \text { out }} \leq 330 \mathrm{~K}, \quad T_{c 2, \text { out }} \leq 300 \mathrm{~K} \\
Q_{F 1} \geq 0.05 \mathrm{~m}^{3} / \mathrm{s}, \quad Q_{M} \geq 0.05 \mathrm{~m}^{3} / \mathrm{s} \\
c_{A, 2} \leq 0.3 \mathrm{kmol} / \mathrm{m}^{3}
\end{array}
$$

The economic objective function is provided by Loeblein and Perkins [1998 on a per unit time basis to maximize the profit calculated based on revenue, and material and utility cost:

$$
\begin{array}{r}
\phi=10\left(Q_{F 1} c_{A, F 1}+Q_{M} c_{A, M}-Q_{2}\left(c_{A, 2}+c_{C, 2}\right)\right) \\
-0.01 q_{c o o l, 1}-q_{c o o l, 2}-0.1 Q_{F 1}-0.1 Q_{M}
\end{array}
$$

The objective function is integrated in the DRTO formulation to account for the accumulated profit throughout the transition. The overall process is decomposed 
into two subunits. The temperature for each CSTR serves as the output for its assigned NMPC. The inlet flow rate to the first CSTR serves as the input for the first NMPC and the flow rate of fresh feed to the mixer for the other NMPC. The safety constraints are directly addressed in the DRTO formulation. The tuning parameters for distributed MPCs and the DRTO formulation are summarized in Table 5.3 .

Table 5.3: DRTO and MPC parameters for the process

\begin{tabular}{lll}
\hline Parameter & Description & Value (MPC1 \& MPC2) \\
\hline$\Delta t_{D R T O}$ & DRTO sample time & $0.5 \mathrm{~s}$ \\
$\Delta t_{M P C}$ & MPC sample time & $0.5 \mathrm{~s}$ \\
$N$ & Optimization horizon & 20 \\
$p$ & MPC Prediction Horizon & 5 \\
$m$ & MPC Control Horizon & 3 \\
$Q$ & Output tracking weight & $1 \& 1$ \\
$R$ & Move suppression weight & $5000 \& 5000$ \\
$y_{\min }, y_{\max }$ & Output bounds & {$[100360] \&[100360]$} \\
$y p_{\min }, y s p_{\max }$ & Set-point bounds & {$[100360] \&[100360]$} \\
$u_{\min }, u_{\max }$ & Input bounds & {$[0.050 .805] \&[0.050 .8]$} \\
\hline
\end{tabular}

Three separate cases of control architecture are compared. The plant is first regulated using the linear MPCs whose assigned set-point trajectories are generated by the DRTO formulation in which a linearized plant model and linear MPC subproblems are used (Case 1). The second case uses a nonlinear plant model in the DRTO formulation to generate the predicted plant response, while retaining the linear formulations for the MPC optimization subproblems and the lower-layer MPCs (Case 2). The expected benefit of this formulation is that the DRTO formulation can take advantage of generating an accurate plant response while maintaining a linear MPC formulation for control actions. The last case implements nonlinear MPC formulations in both lower-layer MPCs and MPC optimization subproblems while the predicted plant outputs are generated with a nonlinear plant model (Case 3). In this way, the dynamics of the lower-level NMPCs can be accurately captured by the closed-loop 
DRTO formulation, and the advantage of accounting for nonlinearity is fully reflected in both levels.

\subsubsection{Target Tracking}

When the values to be achieved for certain outputs of the process are known, the DRTO formulation can be used to drive the system to achieve such targets by judiciously adjusting the set-point trajectories for the lower-level MPCs. In this section, the process objective is to raise the temperatures of the two CSTR tanks by $5 K$. Hence, the objective function for DRTO formulation is expressed in a least squares fashion:

$$
\phi=\Delta t_{\mathrm{MPC}} \sum_{j=1}^{N}\left(T_{1, j}^{\mathrm{DRTO}}-T_{1}^{\mathrm{target}}\right)^{2}+\left(T_{2, j}^{\mathrm{DRTO}}-T_{2}^{\mathrm{target}}\right)^{2}
$$

where $T_{1}^{\text {target }}=347 \mathrm{~K}$ and $T_{2}^{\text {target }}=336 \mathrm{~K}$. The three cases classified previously are each executed under the same objective function. The process responses are shown in Fig. 5.6. From the graphs, it can be easily seen that the set-points are firstly adjusted to the upper bound to induce a faster response of the process and gradually settle down to the target values. These cases show minor variations due to the different levels of nonlinearity brought by the MPC formulations and plant model in the DRTO formulation.

To numerically evaluate the process performance for the three scenarios, the sumsquared-error (SSE) values are calculated, by taking the difference between the final target values and process values at each sample time. The results are summarized in Table. 5.4. The third case has the smallest SSE value, since the lower-level MPCs and MPC optimization subproblems in the DRTO formulation, and plant model used to generated plant responses are all nonlinear and overall can generate the most accurate 

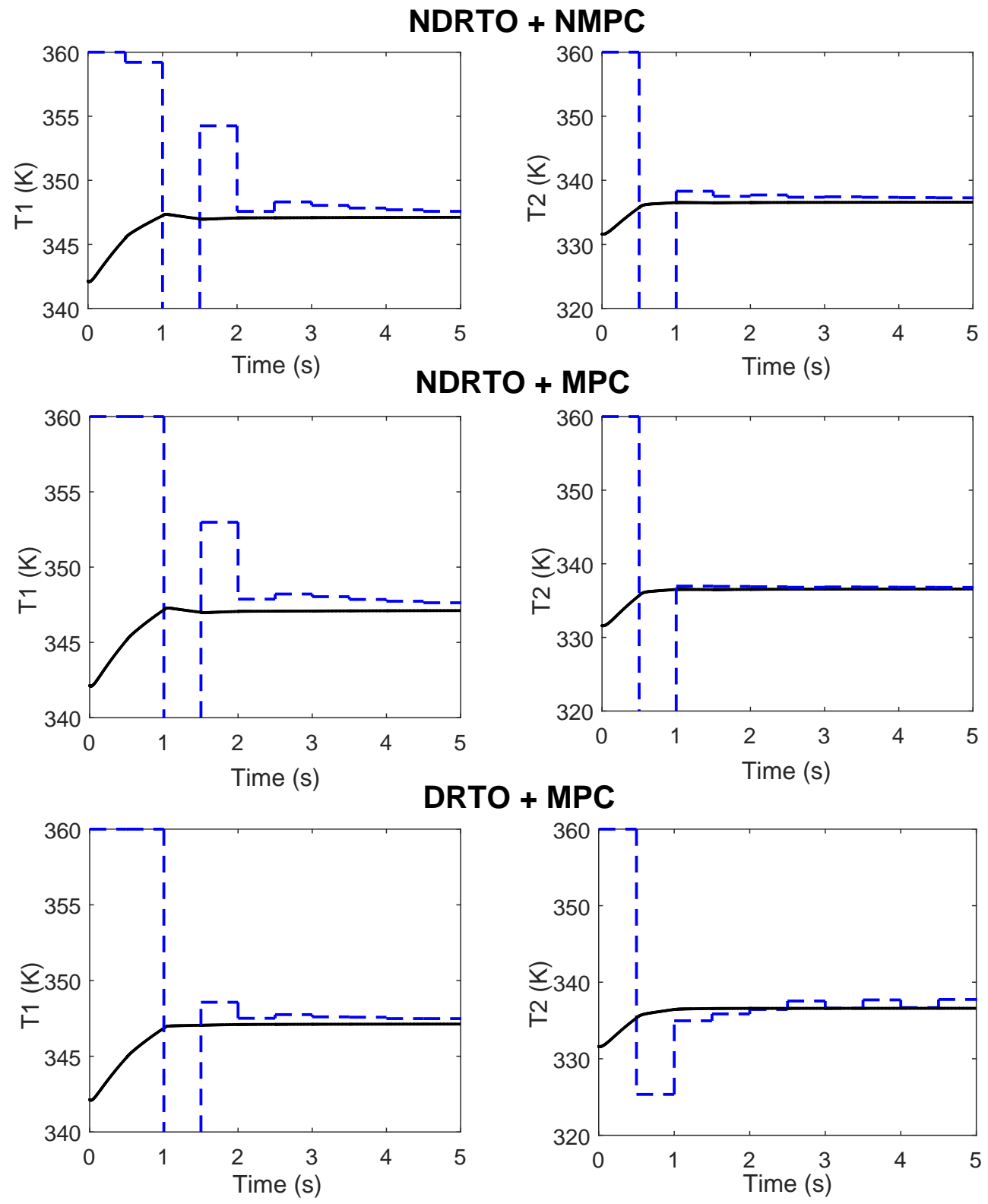

Figure 5.6: The plant response and set-point trajectories for target tracking 
closed-loop predictions and control implementations throughout the simulation.

Table 5.4: The summary of SSE values for three cases

\begin{tabular}{lll}
\hline Case & Description & SSE values \\
\hline 1 & DRTO + MPC & 56.56 \\
2 & NDRTO + MPC & 54.78 \\
3 & NDRTO + NMPC & 53.57 \\
\hline
\end{tabular}

\subsubsection{Economic Optimization}

The process responses for economic optimization for all three cases are illustrated in Fig. 5.7. All three cases achieve the final optimal steady state values specified in Loeblein and Perkins [1998]. However, despite the steady-state optima for all cases being consistent with each other, the process transition can exhibit different behavior. To optimize such transitions is the main goal of the DRTO formulations, and it can be numerically evaluated by calculating the cumulative profits for all cases which are summarized in Table. 5.5.

Table 5.5: The summary of profits for three cases

\begin{tabular}{lll}
\hline Case & Description & $\begin{array}{l}\text { Accumulated } \\
\text { profits }\end{array}$ \\
\hline 1 & DRTO + MPC & 1721.25 \\
2 & NDRTO + MPC & 1721.97 \\
3 & NDRTO + NMPC & 1722.53 \\
\hline
\end{tabular}

The case where the models available for both DRTO and MPC formulations are nonlinear gives the highest profit due to its accuracy of capturing the plant dynamics in the DRTO formulation to generate set-point trajectories and lower-layer MPC control implementations. The differences in profit are relatively small in this application, 
likely due to the small range of the transition between the initial and final operating points. 

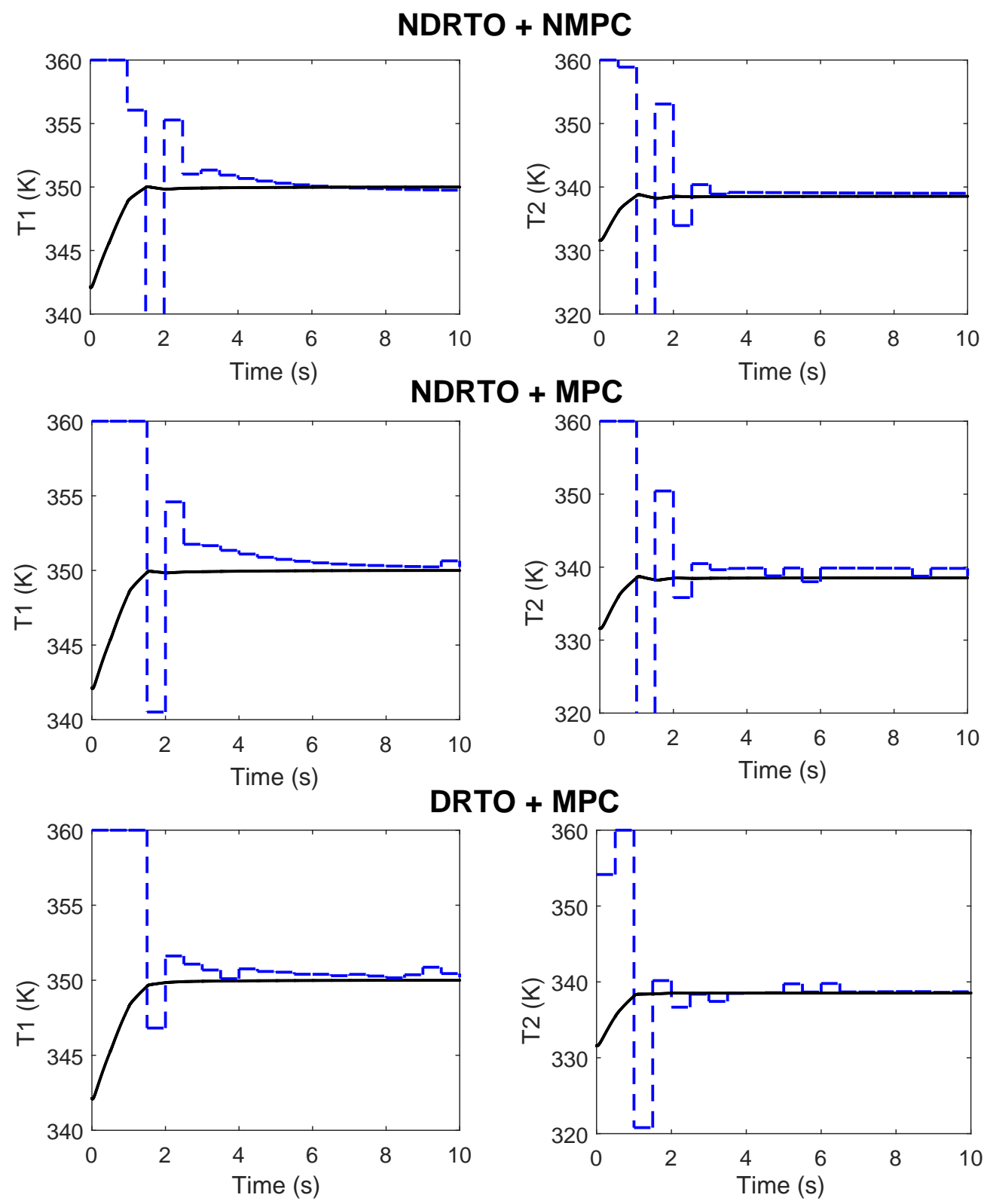

Figure 5.7: The plant response and set-point trajectories for economic optimization 


\section{Chapter 6}

\section{Conclusions and Recommendations}

This chapter summarizes the contributions of the entire study and some recommendations are made for future research directions.

\subsection{Conclusions}

The primary focus of this thesis is to design a coordination scheme for distributed MPCs using the dynamic real-time optimization (DRTO) formulation. This formulation fully considers the interactions between distributed MPCs and the dynamic plant behaviour and complies with the hierarchical industrial process automation control structure.

\section{Closed-loop prediction formulation for distributed MPCs}

The interactions between distributed MPCs and plant dynamics are rigorously modeled in the DRTO formulation. The distributed MPCs only consider the local plant behavior while the plant dynamic model contains the information for the entire plant

process. Such persistent plant model mismatch is simulated along the closed-loop 
prediction horizon. The performance of DRTO formulation is compared with control structures with only centralized or decentralized MPCs. The advantages of using the DRTO formulation to coordinate distributed MPCs are clearly demonstrated using both linear and nonlinear case studies. The capabilities of target tracking and economic optimization are also demonstrated.

\section{Approximation techniques applied to closed-loop prediction for dis- tributed MPC systems}

Due to the complexity of closed-loop prediction for multiple distributed MPCs with plant dynamics, two approximations methods are discussed: the hybrid formulation and the input clipping method. Those approaches significantly reduces the complexity of closed-loop prediction. The performance and problem size analysis are first done on a MIMO linear system. Economic optimization is subsequently conducted on a two-CSTR-in-series system and the computing time can be drastically diminished, which further indicates the practical application of DRTO formulation in a realistic setting.

\section{Inclusion of nonlinearity within the DRTO formulation}

The DRTO formulation introduced previously is based on linear dynamic models for both control implementation and generating surrogate plant outputs. This chapter demonstrates that introducing various degrees of nonlinearity can improve the performance of DRTO formulation since the closed-loop prediction is able to reflect the plant dynamics with better accuracy. Firstly, the holistic plant model takes a nonlinear form and this nonlinear formulation is compared with its linear counterparts. A noticeable increase in performance is observed for the case study observed. Subsequently, the MPCs also utilize a nonlinear formulation to provide a better control implementation in both the lower and upper levels. The performance is further improved based on the case study considered. 


\subsection{Recommendations for Further Work}

Some broad areas for future research in this area related to this topic are summarized:

\section{Large-scale industrial applications of DRTO formulation for distributed MPCs}

A case study should be conducted on a large-scale industrial system so that the benefit of using this hierarchical structure can be fully captured. However, due to the large simulation models used for large-scale systems, embedding the holistic plant model or local models can be challenging. Thus, certain model reduction methods should be properly explored. Due to current industrial applications of steady-state RTO, transforming the steady-state models to a dynamic one could be another solution to this problem. Approximation techniques should be also applied to alleviate the issue of computational complexity.

\section{Alternative MPC formulations used in the control implementation and inner MPC optimization subproblems of DRTO formulation}

The MPC formulation used in this study is limited to stable system dynamics so the issue of stability is largely not explored. Various formulations have been developed in the research area to address the issue of stability. Incorporating some of those formulations would demonstrate the wide applicability of the DRTO formulation for various lower-control structure and for both stable and unstable systems. Rigorous theoretical proofs for stability are also needed for the DRTO formulation to illustrate its ability to handle unstable systems. Various discretization methods for nonlinear MPCs can be another option to explore. Logic-based MPCs are used in industry, and addressing such formulations would expand the potential choices of application as well.

\section{Robust model-based control using closed-loop prediction}


Certain parameters in industrial processes are time varying. Such uncertainty can be addressed using the hierarchical control structure by assigning optimized set-point trajectories by the upper layer to the lower level control implementation. The lowerlevel MPC contains a dynamic model with a nominal value of the uncertain variable. Assuming there is known minimum and maximum of the uncertainty parameter, the upper layer utilizes the closed-loop prediction with multiple realizations of the plant dynamics. Each scenario can produce the closed-loop dynamics between its corresponding plant realization and the nominal MPC. All MPC optimization subproblems track a universal reference trajectory generated by the primary optimization part, and the set-point trajectories are extracted from this reference trajectory for lower-level control tracking. In this way, while maintaining the simple control structure of a nominal MPC, the upper layer effective capture the possible scenarios of constraint violations and closed-loop dynamics. Both target-tracking and economic optimization are feasible when this structure is used. 


\section{List of References}

Allgower, F., Findeisen, R., Nagy, Z. K., et al. (2004). Nonlinear model predictive control: From theory to application. Journal of the Chinese Institute of Chemical Engineers, 35(3), 299-315.

Amrit, R., Rawlings, J. B., And Angeli, D. (2011). Economic optimization using model predictive control with a termional cost. Ann. Rev. Contr., 35, 178186.

Baker, R. And Swartz, C. L. E. (2004). Simultaneous solution strategies for inclusion of input saturation in the optimal design of dynamically operable plants. Optimization and Engineering, 5(1), 5-24.

Baker, R. And Swartz, C. L. E. (2008). Interior point solution of multilevel quadratic programming problems in constrained model predictive control applications. Ind. Eng. Chem. Res., 47(1), 81-91.

Baldea, M. And Daoutidis, P. (2007). Control of integrated process networks A multi-time scale perspective. Computers and Chemical Engineering, 31(5), 426 -444 .

Baumrucker, B., Renfro, J., And Biegler, L. (2008). Mpec problem formulations and solution strategies with chemical engineering applications. Computers E3 Chemical Engineering, 32(12), 2903 - 2913. 
Camponogara, E., Jia, D., Krogh, B. H., and Talukdar, S. (2002). Distributed model predictive control. IEEE Control Syst., 22(1), 44-52.

Chen, X., Heidarinejad, M., Liu, J., and Christofides, P. D. (2012). Distributed economic MPC: Application to a nonlinear chemical porocess network. $J$. Process Control, 22, 689 - 699.

Chien, I.-L., Huang, H.-P., And Yang, J.-C. (1999). A simple multiloop tuning method for PID controllers with no proportional kick. Ind. Eng. Chem. Res., 38(4), $1456-1468$.

Christofides, P. D., Scattolini, R., Muñoz de la Peña, D., and Liu, J. (2013). Distributed model predictive control: A tutorial review and future research directions. Comput. Chem. Eng., 51, $21-41$.

Congalidis, J. P., Richards, J. R., and Ray, W. H. (1986). Modeling and control of a copolymerization reactor. In 1986 American Control Conference, pp. 1779-1793.

Cutler, C. R. And Ramaker, B. L. (1979). Dynamic matrix control - a computer control algorithm. In AIChE 86th National Meeting, Houston, TX, USA.

Darby, M. L., Nikolaou, M., Jones, J., And Nicholson, D. (2011). RTO: An overview and assessment of current practice. J. Process Control, 21(6), 874-884.

Ellis, M. And Christofides, P. D. (2014). Integrating dynamic economic optimization and model predictive control for optimal operation of nonlinear process systems. Control Eng. Pract., 22, 242-251.

Ellis, M., Durand, H., And Christofides, P. D. (2014). A tutorial review of economic model predictive control methods. J. Process Control, 24(8), 1156-1178.

EnGell, S. (2007). Feedback control for optimal process operation. J. Process Control, 17(3), $203-219$. 
Farina, M. And Scattolini, R. (2012). Distributed predictive control: A noncooperative algorithm with neighbor-to-neighbor communication for linear systems. Automatica, 48(6), 1088-1096.

Farina, M., Zhang, X., And Scattolini, R. (2017). A hierarchical MPC scheme for interconnected systems. IFAC-PapersOnLine, 50(1), 12021 - 12026.

Garcia, C. E. And Morshedi, A. M. (1986). Quadratic programming solution of dynamic matrix control (QDMC). Chem. Eng. Commun., 46(1-3), 73-87.

Gudi, R. D. And Rawlings, J. B. (2006). Identification for decentralized model predictive control. AIChE Journal, 52(6), 2198-2210.

Heidarinejad, M., Liu, J., And Christofides, P. D. (2012). Economic model predictive control of nonlinear process systems using lyapunov techniques. AIChE J., 58(3), 855-870.

Jamaludin, M. Z., Li, H., And Swartz, C. L. E. (2017). The utilization of closed-loop prediction for dynamic real-time optimization. Can. J. Chem. Eng., 95, $1968-1978$.

Jamaludin, M. Z. And Swartz, C. L. E. (2016). Closed-loop formulation for nonlinear dynamic real-time optimization. IFAC-PapersOnLine, 49(7), 406 - 411.

Jamaludin, M. Z. And Swartz, C. L. E. (2017a). Approximation of closed-loop prediction for dynamic real-time optimization calculations. Comput. Chem. Eng., 103, $23-38$.

Jamaludin, M. Z. and Swartz, C. L. E. (2017b). Dynamic real-time optimization with closed-loop dynamics. AIChE J., 63(9), 3896 - 3911.

Kadam, J. V., Schlegel, M., Marquardt, W., Tousain, R. L., van Hessem, D. H., van den Berg, J., And Bosgra, O. H. (2002). A two-level strategy 
of integrated dynamic optimization and control of industrial processesa case study. In Grievink, J. and van Schijndel, J. (Eds.), European Symposium on Computer Aided Process Engineering-12, Vol. 10 of Computer Aided Chemical Engineering, pp. 511 - 516. Elsevier.

Liu, J., Muñoz de la Peña, D., And Christofides, P. D. (2009). Distributed model predictive control of nonlinear process systems. AIChE J., 55(5), 1171-1184.

Loeblein, C. And Perkins, J. (1998). Economic analysis of different structures of on-line process optimization systems. Comput. Chem.l Eng., 22(9), 1257 - 1269.

Maciejowski, J. M. (2002). Predictive control with constraints. Pearson education.

Maestre, J. M., Negenborn, R. R., And Editors (2014). Model Predictive Control Made Easy. Springer.

Marcos, N. I., Forbes, J. F., And Guay, M. (2014). Prediction-driven coordination of distributed MPC controllers for linear unconstrained dynamic systems. Int. J. Control, 87(8), 1496-1512.

Marlin, T. E. AND Hrymak, A. N. (1997). Real-time operations optimization of continuous processes. AIChE Symposium Series: Proceedings of the 5th International Conference on Chemical Process Control, 5, 156 - 164.

Mart, R., Sarabia, D., Navia, D., And De Prada, C. (2013). A method to coordinate decentralized nmpc controllers in oxygen distribution networks. Comput. Chem. Eng., 59, $122-137$.

Pannocchia, G. (2013). Distributed model predictive control. Encyclopedia of Systems and Control, , 1-9.

QIN, S. J. AND BADGWELl, T. A. (2003). A survey of industrial model predictive control technology. Control Engineering Practice, 11(7), 733 - 764. 
RAlph, D. AND WRight, S. J. (2004). Some properties of regularization and penalization schemes for MPECs. Optim. Methods Softw., 19(5), 527-556.

Razzanelli, M. And Pannocchia, G. (2016). Parsimonious cooperative distributed MPC for tracking piece-wise constant setpoints. IFAC-PapersOnLine, $49(7), 520-525$.

Scattolini, R. (2009). Architectures for distributed and hierarchical model predictive control a review. J. Process Control, 19(5), $723-731$.

Scheu, H. And Marquardt, W. (2011). Sensitivity-based coordination in distributed model predictive control. J. Process Control, 21(5), 715 - 728.

Stewart, B. T., Venkat, A. N., Rawlings, J. B., Wright, S. J., And Pannocchia, G. (2010). Cooperative distributed model predictive control. Syst. Control Lett., 59(8), $460-469$.

Sun, Y. AND El-FARRA, N. H. (2008). Quasi-decentralized model-based networked control of process systems. Comput. Chem. Eng., 32, 2016 - 2029.

Tosukhowong, T., Lee, J. M., Lee, J. H., And Lu, J. (2004). An introduction to a dynamic plant-wide optimization strategy for an integrated plant. Comput. Chem. Eng., 29(1), 199-208.

Venkat, A. N., Hiskens, I. A., Rawlings, J. B., And Wright, S. J. (2008). Distributed MPC strategies with application to power system automatic generation control. IEEE Trans. Control Syst. Technol., 16(6), 1192-1206.

Würth, L., Hannemann, R., And Marquardt, W. (2011). A two-layer architecture for economically optimal process control and operation. J. Proc. Contr., $\mathbf{2 1}(3), 311-321$.

Zafiriou, E. And Marchal, A. L. (1991). Stability of SISO quadratic dynamic matrix control with hard output constraints. AIChE J., 37(10), 1550-1560. 
